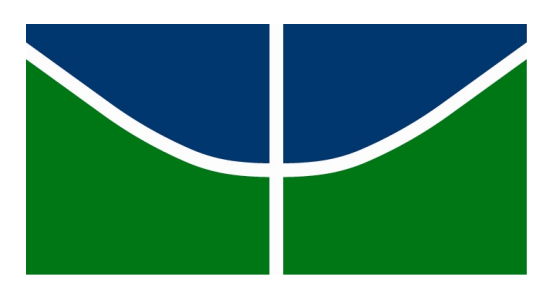

\author{
Universidade de Brasília \\ Instituto de Ciências Exatas \\ Departamento de Matemática
}

\title{
Superálgebras de Lie fractais de crescimento linear
}

\author{
por \\ Otto Augusto de Morais Costa \\ Orientador: Prof. Dr. Victor Petrogradskiy
}


Universidade de Brasília

Instituto de Ciências Exatas

Departamento de Matemática

\section{Superálgebras de Lie fractais de crescimento linear}

por

\section{Otto Augusto de Morais Costa}

Tese apresentada ao Departamento de Matemática da Universidade de Brasília como parte dos requisitos necessários para obtenção do grau de

\section{DOUTOR EM MATEMÁTICA}

07 de dezembro de 2016

Comissão Examinadora:

$\overline{\text { Prof. Dr. Victor Petrogradskiy - Orientador (MAT-UnB) }}$

Prof. Dr. Said Najati Sidki (MAT-UnB)

Prof. Dr. Alexei Krassilnilkov (MAT-UnB)

Prof. Dr. Ivan Shestakov (IME-USP)

Prof. Dr. Plamen Koshlukov (IMECC-UNICAMP) 
Aos meus pais, Osvaldo e Joana D'arc. 


\section{Agradecimentos}

- Ao Deus eterno, pelo amor incondicional e pelo favor de estar comigo todos os dias, até a consumação dos séculos.

- Aos meus pais, Osvaldo e Joana D'arc, por terem me ensinado num bom caminho e pelo grande apoio que têm me dado em todos os momentos.

- À minha irmã Érika, pelo companheirismo de sempre.

- A todos os meus amigos e familiares, por tornarem minha vida mais leve.

- Ao professor Victor Petrogradskiy, meu orientador, por ter acreditado no meu potencial e pelo apoio que me deu ao longo de todo o meu doutorado.

- Ao professor Marco Antonio Pellegrini, meu orientador no mestrado, por ter aberto as portas para a minha entrada no doutorado.

- Aos professores Said Sidki, Alexei Krassilnikov, Ivan Shestakov e Plamen Kochloukov, membros da banca da minha defesa, pelas grandes contribuições prestadas a este trabalho.

- ̇̀ CAPES e ao CNPq, pelo apoio financeiro. 


\section{Resumo}

Os grupos de Grigorchuk e Gupta-Sidki desempenham um papel fundamental na teoria de grupos moderna, pois são exemplos naturais de grupos periódicos finitamente gerados autossimilares. Neste trabalho, construímos exemplos análogos aos grupos referidos no campo das superálgebras de Lie.

Em 2006, Petrogradsky construiu um exemplo análogo para álgebras de Lie restritas em característica 2. Shestakov e Zelmanov estenderam essa contrução para característica positiva arbitrária, dando um exemplo de álgebra de Lie restrita finitamente gerada com p-aplicação nil. Martinez e Zelmanov provaram que, sobre um corpo de característica zero, não é possível construir exemplos de álgebras de Lie análogas aos grupos de Grigorchuk.

Neste trabalho, mostramos que a extensão desse resultado para superálgebras de Lie em característica zero não é válida. Em qualquer característica, construímos uma superálgebra de Lie $\mathbf{R}$ com as seguintes propriedades. $\mathbf{R}$ tem uma $\mathbb{Z}^{2}$-graduação fina tal que todo elemento homogêneo na $\mathbb{Z}_{2}$-graduação $\mathbf{R}=\mathbf{R}_{\overline{0}} \oplus \mathbf{R}_{\overline{1}}$ é ad-nilpotente. Além disso, $\mathbf{R}$ tem crescimento linear e sua envoltória associativa tem crescimento quadrático. Mostramos também que a superálgebra de Lie $\mathbf{R}$ é just infinite.

Para uma característica positiva arbitrária $p$, construímos também um exemplo de álgebra de Lie restrita fractal de crescimento linear cuja envoltória associativa possui crescimento quadrático.

Palavras-chave: superálgebras de Lie, álgebras de Lie restritas, crescimento, álgebras nil, álgebras autossimilares, álgebras graduadas. 


\section{Abstract}

The Grigorchuk and Gupta-Sidki groups play fundamental role in modern group theory because they are natural examples of self-similar finitely generated periodic groups. In this work we construct their analogue in the world of Lie superalgebras.

In 2006, Petrogradsky made an analogous construction for restricted Lie algebras in characteristic 2. Next, Shestakov and Zelmanov extended this construction to an arbitrary positive characteristic, giving an example of finitely generated restricted Lie algebra with a nil $p$-mapping. Martinez and Zelmanov proved that similar examples do not exist for Lie algebras in characteristic zero.

In this work we show that an extension of this result for Lie superalgebras in characteristic zero is not valid. In case of an arbitrary characteristic, we construct a Lie superalgebra $\mathbf{R}$ with the following properties. We prove that $\mathbf{R}$ has a fine $\mathbb{Z}^{2}$-gradation and all homogeneous elements of the gradation $\mathbf{R}=\mathbf{R}_{\overline{0}} \oplus \mathbf{R}_{\overline{1}}$ are ad-nil. Furthermore, $\mathbf{R}$ has linear growth and its associative hull has a quadratic growth.

For an arbitrary positive characteristic $p$, we also construct an example of a fractal restricted Lie algebra of linear growth and such that its associative hull has a quadratic growth.

Keywords: Lie superalgebras, restricted Lie algebras, growth, nil algebras, self-similar algebras, graded algebras. 


\section{Sumário}

1 Introdução $\quad 9$

1.1 Álgebras e grupos de Golod-Shafarevich . . . . . . . . . . . . . . . 9

1.2 Grupos de Grigorchuk e Gupta-Sidki . . . . . . . . . . . . . . . . . . . 10

1.3 Álgebras associativas nil graduadas autossimilares . . . . . . . . . . . 10

1.4 Álgebras de Lie restritas nil . . . . . . . . . . . . . . . . . . 11

1.5 Álgebras de Lie sobre um corpo de característica zero . . . . . . . . . 13

1.6 Superálgebras de Lie nil graduadas fractais . . . . . . . . . . . . . . 13

1.7 Álgebras de Lie restritas nil de crescimento lento . . . . . . . . . . . . . 14

2 Definições básicas $\quad 16$

2.1 Álgebras de Lie, álgebras de Lie restritas e suas envelopantes . . . . . . 16

2.2 Álgebras graduadas . . . . . . . . . . . . . . . . . . . . . . . . . . 21

2.3 Superálgebras de Lie . . . . . . . . . . . . . . . . . . 21

2.4 Crescimento de álgebras . . . . . . . . . . . . . . . . . . . . . . . . . . . 24

2.5 Álgebra de Lie das derivações especiais . . . . . . . . . . . . . . . . . . 26

2.6 Superálgebra de Lie das superderivações $\ldots$. . . . . . . . . . . . . . . . 26

$\begin{array}{llr}3 & \text { Resultados principais } & 29\end{array}$

3.1 A superálgebra de Lie $\mathbf{R} \ldots \ldots \ldots$. . . . . . . . . . . . . . . . 29

3.2 A álgebra de Lie restrita $\mathbf{R} \ldots \ldots \ldots$. . . . . . . . . . . . . . 31 
4 A superálgebra de Lie R 33

4.1 Relações principais . . . . . . . . . . . . . . . . . . 33

4.2 Base de $\mathbf{R} \ldots \ldots \ldots \ldots \ldots \ldots$

4.3 Funções peso . . . . . . . . . . . . . . . . . . . . . 41

$4.4 \mathbb{Z}^{2}$-graduação de $\mathbf{R}$; coordenadas peso e multigrau . . . . . . . . . . . . 42

4.5 Limitações para os pesos . . . . . . . . . . . . . . . . . . . . . . 47

4.6 Crescimento de $\mathbf{R}$ e $\mathbf{A} \ldots \ldots \ldots \ldots \ldots$. . . . . . . . . . . . . . 51

4.7 Funções geradoras de $\mathbf{R} \ldots \ldots$. . . . . . . . . . . . . . . . . . . 60

4.8 Ad-nilpotência dos elementos homogêneos da superálgebra de Lie $\mathbf{R}=$

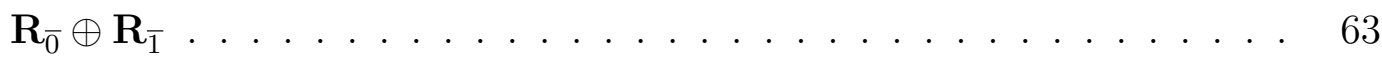

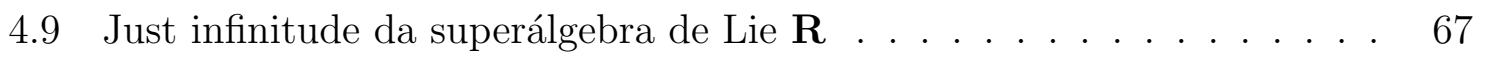

$\begin{array}{lll}5 & \text { A álgebra de Lie restrita } R & 70\end{array}$

5.1 Relações principais . . . . . . . . . . . . . . . . . . 70

5.2 Bases de $\mathbf{L}$ e $\mathbf{R} \ldots \ldots \ldots \ldots \ldots$. . . . . . . . . . . . . . . . . 74

5.3 Funções peso . . . . . . . . . . . . . . . . . . . . . . . . . . . . . 77

$5.4 \mathbb{Z}^{2}$-graduação de $\mathbf{R}$; coordenadas peso e multigrau . . . . . . . . . . . . 79

5.5 Estimativas para os pesos . . . . . . . . . . . . . 82

5.6 Crescimento de $\mathbf{R}$ e $\mathbf{A} \ldots \ldots \ldots \ldots \ldots$. . . . . . . . . . . . . 84

5.7 As componentes homogêneas da $\mathbb{Z}^{2}$-graduação de $\mathbf{R}$ são no máximo unidimensionais . . . . . . . . . . . . . . . . . . . . . 92

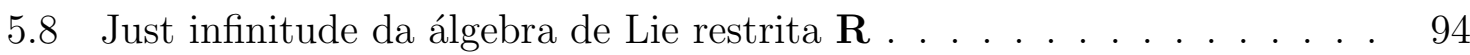




\section{Capítulo 1}

\section{Introdução}

Neste capítulo, faremos um levantamento de alguns resultados conhecidos, os quais servem de motivação para a construção dos nossos exemplos.

\section{1 Álgebras e grupos de Golod-Shafarevich}

O Problema Geral de Burnside apresenta a seguinte questão: um grupo periódico finitamente gerado é necessariamente finito? Golod e Shafarevich provaram que a resposta para tal questão é negativa, mostrando que para cada primo $p$ existe um $p$ grupo finitamente gerado infinito $[13,14]$. A construção desses grupos baseia-se em uma famosa construção de uma família de álgebras associativas nil finitamente geradas de dimensão infinita [13]. Essa construção também fornece exemplos de álgebras de Lie $L$ finitamente geradas de dimensão infinita sobre um corpo arbitrário tais que $(\operatorname{ad} x)^{n(x, y)}(y)=0$ para quaisquer $x, y \in L[15]$. Sendo o corpo de característica positiva $p$, obtém-se uma álgebra de Lie restrita $L$ finitamente gerada de dimensão infinita cuja $p$-aplicação é nil, ou seja, $x^{\left[p^{n(x)}\right]}=0$ para todo $x \in L$. Isso dá uma resposta negativa para uma questão de Jacobson que indaga se uma álgebra de Lie restrita $L$ finitamente gerada é necessariamente finita quando cada elemento $x \in L$ é algébrico, isto é, quando cada $x \in L$ é raiz de algum $p$-polinômio $f_{p, x}(x)=a_{0} x^{\left[p^{m}\right]}+a_{1} x^{\left[p^{m-1}\right]}+\cdots+a_{m} x$ ([21, Ch. 5, ex. 17]). Sabe-se que a construção de Golod fornece álgebras associativas nil de crescimento exponencial. Usando relações especialmente escolhidas, Lenagan e Smoktunowicz construíram álgebras associativas nil de crescimento polinomial [27]. Para obter um maior aprofundamento acerca das álgebras e dos grupos de GolodShafarevich, veja $[50,11]$. 
Uma construção diferente foi motivada por resultados respectivos da teoria de grupos. Uma álgebra de Lie restrita $G$ é dita larga se existe uma subálgebra $H \subset G$ de codimensão finita tal que $H$ admite um homomorfismo sobrejetivo sobre uma álgebra de Lie restrita livre não abeliana. Seja $K$ um corpo perfeito enumerável de característica positiva. Então existem álgebras de Lie restritas nil finitamente geradas de dimensão infinita sobre $K$ que são residualmente finitas e limites diretos de álgebras de Lie restritas largas [3].

\subsection{Grupos de Grigorchuk e Gupta-Sidki}

A construção feita por Golod é um tanto indireta. Grigorchuk, entretanto, fez uma construção direta e elegante de um 2-grupo infinito gerado por três elementos de ordem 2 [16]. Esse grupo foi definido como um grupo de transformações do intervalo [0,1] do qual são removidos números racionais da forma $\left\{k / 2^{n} \mid 0 \leq k \leq 2^{n}, n \geq 0\right\}$. Para cada primo $p \geq 3$, Gupta e Sidki fizeram uma construção direta de um $p$-grupo infinito com dois geradores de ordem $p$ [19]. Esse grupo foi construído como um subgrupo de um grupo de automorfismos de uma árvore regular infinita de grau $p$.

Os grupos de Grigorchuk e Gupta-Sidki são contraexemplos para o Problema Geral de Burnside. Além disso, eles dão respostas para problemas importantes na teoria de grupos. O grupo de Grigorchuk e suas generalizações são os primeiros exemplos de grupos de crescimento intermediário [17], os quais dão uma resposta negativa para uma conjectura de Milnor de que grupos de crescimento intermediário não existem. A construção de Gupta-Sidki também resulta em grupos de crescimento subexponencial [12]. Os grupos de Grigorchuk e Gupta-Sidki são autossimilares. Em particular, eles contêm infinitas cópias de si mesmos. Os grupos autossilimares, em especial os denominados grupos de ramificação, constituem uma área bem estabelecida na teoria de grupos. Para um aprofundamento maior, veja [18]. A seguir, discutiremos a existência de análogos aos grupos de Grigorchuk e Gupta-Sidki para outras estruturas algébricas.

\section{3 Álgebras associativas nil graduadas autossimi- lares}

O estudo desses grupos levou a uma investigação acerca de anéis de grupos e outras álgebras associativas relacionadas [46]. Em particular, surgiram álgebras associativas 
autossimilares definidas por matrizes de maneira recorrente [5]. As matrizes recorrentes foram também aplicadas em problemas da teoria dos números [1]. Sidki sugeriu dois exemplos de álgebras de matrizes associativas autossimilares [45]. Uma família mais geral de álgebras associativas autossimilares foi introduzida em [37]. Essa família generaliza o segundo exemplo de Sidki [45] e, através dela, é possível obter exemplos de álgebras de Lie restritas de Fibonacci (veja abaixo) em termos de matrizes autossimilares [37]. Outra característica importante de algumas álgebras associativas autossimilares $A$ construídas em [37] é que elas são somas de duas subálgebras localmente nilpotentes $A=A_{+} \oplus A_{-}$. Recordemo-nos de que uma álgebra é dita localmente nilpotente se toda subálgebra finitamente gerada é nilpotente.

Mas os análogos desejados dos grupos de Grigorchuk e Gupta-Sidki devem ser álgebras associativas autossimilares nil, de modo a produzir novos exemplos de grupos periódicos finitamente gerados. Mas tais exemplos ainda não são conhecidos. Problemas em aberto na teoria de álgebras de dimensão infinita podem ser vistos em [51].

\section{4 Álgebras de Lie restritas nil}

Ao contrário das álgebras associativas, para álgebras de Lie restritas existem análogos naturais dos grupos de Grigorchuk e Gupta-Sidki. Sobre um corpo de característica 2, Petrogradsky construiu um exemplo de uma álgebra de Lie restrita de dimensão infinita $\mathbf{L}$ gerada por dois elementos, denominada álgebra de Lie restrita de Fibonacci [33]. Seja char $K=2$ e seja $R=K\left[t_{i} \mid i \geq 0\right] /\left(t_{i}^{p} \mid i \geq 0\right)$ um anel de polinômios truncado. Fazendo $\partial_{i}=\frac{\partial}{\partial t_{i}}, i \geq 0$, são definidas duas derivações de $R$ :

$$
\begin{array}{lrr}
v_{1}= & \partial_{1}+t_{0}\left(\partial_{2}+t_{1}\left(\partial_{3}+t_{2}\left(\partial_{4}+t_{3}\left(\partial_{5}+t_{4}\left(\partial_{6}+\cdots\right)\right)\right)\right)\right) ; \\
v_{2}= & \partial_{2}+t_{1}\left(\partial_{3}+t_{2}\left(\partial_{4}+t_{3}\left(\partial_{5}+t_{4}\left(\partial_{6}+\cdots\right)\right)\right)\right) .
\end{array}
$$

Essas duas derivações geram uma álgebra de Lie restrita $\mathbf{L}=\operatorname{Lie}_{p}\left(v_{1}, v_{2}\right) \subset$ Der $R$ e uma álgebra associativa $\mathbf{A}=\operatorname{Alg}\left(v_{1}, v_{2}\right) \subset$ End $R$. Bergman mostrou que a dimensão de Gelfand-Kirillov de uma álgebra associativa não pode pertencer ao intervalo $(1,2)[23]$. Isso, porém, não é válido para álgebras de Lie. A dimensão de Gelfand-Kirillov de uma álgebra de Lie finitamente gerada pode ser qualquer número pertencente ao conjunto $\{0\} \cup[1,+\infty][32]$. A álgebra de Lie de Fibonacci tem um lento crescimento polinomial, com dimensão de Gelfand-Kirillov GKdim $\mathbf{L}=\log _{\frac{\sqrt{5}+1}{2}} 2 \approx 1.44$ [33]. A álgebra de Lie restrita $\mathbf{L}$ é autossimilar. Outras propriedades da álgebra de Lie restrita de Fibonacci e suas generalizações são estudadas em [36, 38]. 
Uma propriedade muito interessante de L é que ela possui uma p-aplicação nil [33], propriedade análoga à periodicidade dos grupos de Grigorchuk e Gupta-Sidki. Não se sabe se a envoltória associativa $\mathbf{A}$ de $\mathbf{L}$ é uma álgebra nil. Entretanto, Petrogradsky e Shestakov provaram uma afirmação mais fraca. As álgebras L e A são somas diretas de duas subálgebras localmente nilpotentes [36]:

$$
\mathbf{L}=\mathbf{L}_{+} \oplus \mathbf{L}_{-}, \quad \mathbf{A}=\mathbf{A}_{+} \oplus \mathbf{A}_{-}
$$

Existem exemplos de álgebras associativas de dimensão infinita que são somas diretas de duas subálgebras localmente nilpotentes [25, 9]. Álgebras de Lie restritas de dimensão infinita podem possuir várias decomposições como somas diretas de duas subálgebras localmente nilpotentes [39].

No caso de uma característica prima arbitrária, Shestakov e Zelmanov sugeriram um exemplo de uma álgebra de Lie restrita finitamente gerada com uma p-aplicação nil [44]. Nesse exemplo, a mesma decomposição (1.1) é obtida para alguns primos $p[26,37]$.

Um exemplo de álgebra de Lie restrita nil $p$-gerada $L$ para uma característica prima $p$ arbitrária foi estudado em [39]. O mérito desse exemplo é que para quaisquer primos temos a mesma decomposição (1.1) em somas diretas de duas subálgebras localmente nilpotentes. Contudo, é um tanto complicado efetuar computações para esses exemplos.

Observe que apenas o exemplo original tem uma base monomial nítida [33, 36]. Em outros exemplos, os elementos da álgebra de Lie são combinações lineares de monômios. Por vezes, trabalhar com tais combinações lineares envolve uma dificuldade técnica considerável $[44,39]$. Uma família contínua de álgebras de Lie restritas nil de crescimento lento com bases monomiais satisfatórias é construída em [40].

Seja $G$ um grupo e $G=G_{1} \supseteq G_{2} \supseteq \cdots$ sua série central inferior. Associada ao grupo $G$, é construída uma álgebra de Lie $\mathbb{Z}$-graduada $L_{K}(G)=\underset{i \geq 1}{\oplus} L_{i}$, onde $L_{i}=G_{i} / G_{i+1} \otimes_{\mathbb{Z}} K$, $i \geq 1$, e char $K=p$. O produto é dado por $\left[a_{i} G_{i+1}, b_{j} G_{j+1}\right]=\left(a_{i}, b_{j}\right) G_{i+j+1}$, onde $\left(a_{i}, b_{j}\right)=a_{i}^{-1} b_{j}^{-1} a_{i} b_{j}$ é o comutador dos elementos $a_{i}, b_{j}$ no grupo $G$. Um residualmente $p$-grupo $G$ é dito de largura finita se todos os fatores $G_{i} / G_{i+1}$ são grupos finitos com ordens uniformemente limitadas.

O grupo de Grigorchuk $G$ é de largura finita. Mais precisamente, foi provado que $\operatorname{dim}_{\mathbb{F}_{2}} G_{i} / G_{i+1} \in\{1,2\}$ para $i \geq 2[43,7]$. Em particular, a respectiva álgebra de Lie $L=L_{K}(G)=\underset{i \geq 1}{\oplus} L_{i}$ tem crescimento linear. Bartholdi provou recentemente que a álgebra de Lie restrita $L_{\mathbb{F}_{2}}(G)$ é nil, ao passo que $L_{\mathbb{F}_{4}}(G)$ não é nil [6]. 


\section{5 Álgebras de Lie sobre um corpo de carac- terística zero}

No caso em que o corpo é de característica zero, não existe um exemplo similar de crescimento lento, devido ao próximo resultado. Em outras palavras, não existem análogos naturais dos grupos de Grigorchuk no campo das álgebras de Lie de característica zero. A afirmação exata é dada no seguinte

Teorema 1.1 (Martinez e Zelmanov [28]) Seja $L=\underset{\alpha \in \Gamma}{\oplus} L_{\alpha}$ uma álgebra de Lie sobre um corpo $K$ de característica zero, graduada por um grupo abeliano $\Gamma$. Suponha que sejam satisfeitas as seguintes condições:

(i) existe $d>0$ tal que $\operatorname{dim}_{K} L_{\alpha} \leq d$ para todo $\alpha \in \Gamma$;

(ii) todo elemento homogêneo $a \in L_{\alpha}, \alpha \in \Gamma$, é ad-nilpotente.

Então L é localmente nilpotente.

\subsection{Superálgebras de Lie nil graduadas fractais}

A investigação acerca da construção de exemplos análogos aos grupos de Grigorchuk e Gupta-Sidki não ficou restrita aos campos das álgebras associativas e das álgebras de Lie. Petrogradsky construiu exemplos de superálgebras de Lie análogas a esses grupos em característica arbitrária [34]. Esses exemplos são também análogos à álgebra de Lie restrita de Fibonacci e a outras álgebras de Lie autossimilares mencionadas acima. Nas superálgebras de Lie construídas, os elementos homogêneos com respeito à $\mathbb{Z}_{2^{-}}$ graduação são ad-nilpotentes. Essa propriedade é análoga à periodicidade dos grupos de Grigorchuk e Gupta-Sidki. Ambas as superálgebras são autossimilares e, portanto, contêm infinitas cópias de si mesmas. Devido a essa propriedade, dizemos que elas são fractais. A construção do primeiro exemplo de [34] é descrita abaixo.

Seja $\Lambda=\Lambda\left[x_{i}, y_{i} \mid i \geq 0\right]$ uma álgebra de Grassmann nas infinitas variáveis $x_{i}, y_{i}, i \geq 0$. Considere os seguintes elementos ímpares em $\mathbf{W}(\Lambda)$ :

$$
\begin{aligned}
& a_{i}=\partial_{x_{i}}+y_{i} x_{i}\left(\partial_{x_{i+1}}+y_{i+1} x_{i+1}\left(\partial_{x_{i+2}}+y_{i+2} x_{i+2}\left(\partial_{x_{i+3}}+\cdots\right)\right)\right), \quad \\
& b_{i}=\partial_{y_{i}}+x_{i} y_{i}\left(\partial_{y_{i+1}}+x_{i+1} y_{i+1}\left(\partial_{y_{i+2}}+x_{i+2} y_{i+2}\left(\partial_{y_{i+3}}+\cdots\right)\right)\right),
\end{aligned} \quad i \geq 0 .
$$


É definida a superálgebra de Lie $\mathbf{R}=\operatorname{Lie}\left(a_{0}, b_{0}\right) \subset \mathbf{W}(\Lambda)$ e sua envoltória associativa $\mathbf{A}=\operatorname{Alg}\left(a_{0}, b_{0}\right) \subset \operatorname{End} \Lambda$. No caso em que char $K=2$, é assumido que a aplicação quadrática nos elementos ímpares coincide com o operador respectivo em End $\Lambda$. A superálgebra $\mathbf{R}$ descrita acima é autossimilar e possui crescimento lento (GKdim $\mathbf{R} \approx$ 1.44). Além disso, $\mathbf{R}=\mathbf{R}_{\overline{0}} \oplus \mathbf{R}_{\overline{1}}$ é nil graduada.

Descreveremos agora o segundo exemplo construído em [34]. Seja $\Lambda=\Lambda\left[x_{i}, y_{i}, z_{i} \mid i \geq\right.$ 0] uma álgebra de Grassmann e considere os seguintes elementos ímpares em $\mathbf{W}(\Lambda)$ :

$$
\begin{aligned}
& a_{i}=\partial_{x_{i}}+y_{i} x_{i}\left(\partial_{x_{i+1}}+y_{i+1} x_{i+1}\left(\partial_{x_{i+2}}+y_{i+2} x_{i+2}\left(\partial_{x_{i+3}}+\cdots\right)\right)\right), \\
& b_{i}=\partial_{y_{i}}+z_{i} y_{i}\left(\partial_{y_{i+1}}+z_{i+1} y_{i+1}\left(\partial_{y_{i+2}}+z_{i+2} y_{i+2}\left(\partial_{y_{i+3}}+\cdots\right)\right)\right), \quad i \geq 0 . \\
& c_{i}=\partial_{z_{i}}+x_{i} z_{i}\left(\partial_{z_{i+1}}+x_{i+1} z_{i+1}\left(\partial_{z_{i+2}}+x_{i+2} z_{i+2}\left(\partial_{z_{i+3}}+\cdots\right)\right)\right),
\end{aligned}
$$

É definida a superálgebra $\mathbf{Q}=\operatorname{Lie}\left(a_{0}, b_{0}, c_{0}\right) \subset \mathbf{W}(\Lambda)$ e sua envoltória associativa $\mathbf{A}=\operatorname{Alg}\left(a_{0}, b_{0}, c_{0}\right) \subset \operatorname{End} \Lambda$. Para char $K=2$, é assumido que a aplicação quadrática nos elementos ímpares coincide com o operador respectivo em End $\Lambda$. A superálgebra $\mathbf{Q}$ descrita acima também é autossimilar e possui crescimento lento ( GKdim $\mathbf{Q} \approx 1.89$ ). Além disso, $\mathbf{Q}=\mathbf{Q}_{\overline{0}} \oplus \mathbf{Q}_{\overline{1}}$ é nil graduada e possui uma $\mathbb{Z}^{3}$-graduação fina pelo multigrau em relação aos geradores $\left\{a_{0}, b_{0}, c_{0}\right\}$. Esse exemplo mostra que não é possível estender o Teorema 1.1 para superálgebras de Lie, pois trata-se de uma superálgebra de Lie não localmente nilpotente, com uma $\mathbb{Z}^{3}$-graduação cujas componentes homogêneas têm dimensões uniformemente limitadas por 1, e cujos elementos homogêneos são adnilpotentes.

\section{7 Álgebras de Lie restritas nil de crescimento lento}

Para um corpo de característica positiva arbitrária, Petrogradsky construiu uma família $\mathbf{L}(\Xi)$ de álgebras de Lie restritas 2-geradas de lento crescimento polinomial com uma p-aplicação nil, onde $\Xi$ é uma infinita tupla de inteiros positivos [40]. A saber, $\operatorname{GKdim} \mathbf{L}(\Xi) \leq 2$ para todas as tais álgebras. Essas álgebras são construídas em termos de derivações de uma álgebra de infinitas potências divididas $\Omega$. A álgebra de Lie $\mathbf{L}$ e sua envoltória associativa $\mathbf{A} \subset \operatorname{End}(\Omega)$ são $\mathbb{Z}^{2}$-graduadas pelo multigrau em relação aos geradores. Se a tupla $\Xi$ é periódica, então $\mathbf{L}(\Xi)$ é autossimilar.

Como caso particular, foi construída uma subfamília contínua de álgebras de Lie restritas nil não isomórficas $\mathbf{L}\left(\Xi_{\alpha}\right), \alpha \in \mathbb{R}^{+}$, com crescimento extremamente lento. A saber, 
elas possuem dimensão de Gelfand-Kirillov igual a 1, mas o crescimento não é linear. Para essa subfamília, as envoltórias associativas A têm dimensão de Gelfand-Kirillov 2, mas o crescimento não é quadrático. A virtude desses exemplos é que eles possuem bases monomiais explícitas. 


\section{Capítulo 2}

\section{Definições básicas}

Neste capítulo, vamos introduzir alguns conceitos básicos. Dentre outras coisas, definiremos superálgebras de Lie e álgebras de Lie restritas, que são os objetos principais deste trabalho. Nos nossos exemplos, o corpo base será denotado por $K$. Utilizaremos a notação $\langle X\rangle_{K}$ para o subespaço linear gerado por um subconjunto $X$ de um espaço vetorial sobre $K$.

\section{1 Álgebras de Lie, álgebras de Lie restritas e suas envelopantes}

Definição 2.1 Uma álgebra de Lie $L$ é um espaço vetorial sobre um corpo $K$ munido de uma operação bilinear $[]:, L \times L \rightarrow L$, denominada colchete de Lie, satisfazendo as seguintes condições, para quaisquer $x, y, z \in L$ :

(i) $[x, y]=-[y, x]$ (anticomutatividade);

(ii) $[x,[y, z]]+[y,[z, x]]+[z,[x, y]]=0$ (identidade de Jacobi).

Quando char $K \neq 2$, a anticomutatividade do colchete de Lie implica que $[x, x]=0$ para todo $x \in L$. No caso em que char $K=2$, adicionamos essa condição aos axiomas de álgebra de Lie. Neste trabalho, colchetes de grandes comprimentos serão normados à direita: $[x, y, z]=[x,[y, z]]$. Para cada $x \in L$, definimos o operador adjunto ad $x$ : $L \rightarrow L$ por ad $x(y)=[x, y]$. Se $(\operatorname{ad} x)^{n}=0$ para algum inteiro positivo $n$, dizemos que o elemento $x \in L$ é ad-nilpotente. 
Seja $A$ uma álgebra associativa. Definindo em $A$ o produto comutador

$$
[x, y]=x y-y x, \quad x, y \in A,
$$

o espaço vetorial $A$ torna-se uma álgebra de Lie, denotada por $A^{(-)}$. Por outro lado, podemos associar a cada álgebra de Lie $L$ uma álgebra associativa com identidade, denominada álgebra envelopante universal.

Definição 2.2 Seja L uma álgebra de Lie sobre um corpo K. Uma álgebra envelopante universal de $L$ é um par $(U, i)$, onde $U$ é uma álgebra associativa unitária sobre $K$ e $i: L \rightarrow U^{(-)}$é um homomorfismo de álgebras de Lie com a seguinte propriedade universal: se A é uma álgebra associativa unitária qualquer sobre $K$ e $\sigma: L \rightarrow A^{(-)}$é um homomorfismo de álgebras de Lie, então existe um único homomorfismo de álgebras associativas $\phi: U \rightarrow A$ tal que $\phi\left(1_{U}\right)=1_{A}$ e $\phi \circ i=\sigma$.

Para toda álgebra de Lie $L$, um par $(U, i)$ como na definição acima existe e é único, a menos de isomorfismos. A prova desse fato pode ser encontrada em [20]. Obteremos a seguir uma construção para $(U, i)$ utilizando o conceito de álgebra tensorial. Primeiramente, vamos definir o produto tensorial de espaços vetoriais.

Definição 2.3 O produto tensorial entre dois espaços vetoriais $A$ e $B$ sobre um mesmo corpo $K$ é um espaço vetorial $A \otimes B$ sobre $K$ com uma aplicação bilinear

$$
\begin{aligned}
\otimes: A \times B & \longrightarrow A \otimes B \\
(a, b) & \longmapsto(a \otimes b)
\end{aligned}
$$

que satisfaz a seguinte condição: se $\left\{a_{i} \mid i \in I\right\}$ e $\left\{b_{j} \mid j \in J\right\}$ são bases de $A$ e $B$, respectivamente, então $\left\{a_{i} \otimes b_{j} \mid i \in I, j \in J\right\}$ é uma base de $A \otimes B$.

A partir da definição acima, podemos introduzir o conceito de produto tensorial de álgebras.

Definição 2.4 Sejam $A$ e $B$ duas álgebras sobre um mesmo corpo K. O produto tensorial $A \otimes B$ é a álgebra cujo espaço é o produto tensorial dos espaços $A$ e $B$, e o produto é definido por

$$
\left(a_{1} \otimes b_{1}\right) \cdot\left(a_{2} \otimes b_{2}\right)=a_{1} a_{2} \otimes b_{1} b_{2}, \quad a_{i} \in A, b_{i} \in B
$$


Seja $A$ uma álgebra qualquer (não necessariamente unitária) sobre um corpo $K$. Consideremos $T^{0}(A)=K$ e $T^{1}(A)=A$. Para $n \geq 2$, denotemos por $T^{n}(A)=A \otimes \cdots \otimes A$ o produto tensorial entre $n$ cópias de uma álgebra $A$.

Definição 2.5 Seja A uma álgebra sobre um corpo $K$. A álgebra tensorial de $A$ é a álgebra associativa unitária $T(A)=\bigoplus_{n=0}^{\infty} T^{n}(A)$.

Através do conceito de álgebra tensorial, podemos obter uma álgebra envelopante universal $(U, i)$ de uma álgebra de Lie $L$ sobre um corpo $K$. Considere o quociente $U(L)=T(L) /(x \otimes y-y \otimes x-[x, y] \mid x, y \in L)$, onde $T(L)$ é a álgebra tensorial do espaço vetorial $L$. Se $\pi: T(L) \rightarrow U(L)$ é o homomorfismo canônico e $i=\left.\pi\right|_{L}: L \rightarrow U(L)$ é a sua restrição a $L$, então o par $(U(L), i)$ constitui uma álgebra envelopante universal para $L$. Para mais detalhes, veja [20].

Observe que, da forma como construímos a álgebra envelopante $U(L)$, o produto considerado em $L$ coincide com o comutador em $U(L)^{(-)}$. O teorema abaixo nos fornece uma base para a álgebra envelopante universal $U(L)$ a partir de uma base ordenada arbitrária da álgebra de Lie $L$.

Teorema 2.6 (Poincaré-Birkhoff-Witt) Para qualquer base $\left\{x_{i} \mid i \in I\right\}$ de uma álgebra de Lie L, onde I é um conjunto ordenado de índices, os monômios

$$
\left\{x_{i_{1}} \cdots x_{i_{n}} \mid i_{1} \leq \ldots \leq i_{n}, n \geq 0\right\}
$$

constituem uma base para a álgebra envelopante $U(L)$.

O Teorema de Poincaré-Birkhoff-Witt, por vezes, é referido abreviadamente por PBW.

Definição 2.7 Uma álgebra de Lie L sobre um corpo $K$ de característica $p>0$ é dita uma álgebra de Lie restrita (ou p-álgebra de Lie) se em L está definida uma operação unária $x \mapsto x^{[p]}, x \in L$, denominada p-aplicação, satisfazendo os seguintes axiomas:

(i) $(\lambda x)^{[p]}=\lambda^{p} x^{[p]}$, para quaisquer $\lambda \in K, x \in L$;

(ii) $\operatorname{ad}\left(x^{[p]}\right)=(\operatorname{ad} x)^{p}$, para todo $x \in L$;

(iii) $(x+y)^{[p]}=x^{[p]}+y^{[p]}+\sum_{i=1}^{p-1} s_{i}(x, y)$, para quaisquer $x, y \in L$, onde $i s_{i}(x, y)$ é $o$ coeficiente de $t^{i-1}$ no polinômio $\operatorname{ad}(t x+y)^{p-1}(x) \in L[t]$. 
Esse conceito é motivado pelo seguinte fato: se $A$ é uma álgebra associativa sobre um corpo $K$ de característica $p>0$, então a aplicação $x \mapsto x^{p}, x \in A^{(-)}$, satisfaz os três axiomas acima.

A expressão dada no axioma (iii) da Definição 2.7 é de difícil aplicabilidade. Portanto, para as nossas computações, utilizaremos a seguinte versão:

$$
(x+y)^{[p]}=x^{[p]}+y^{[p]}+(\operatorname{ad} y)^{p-1}(x)+\sum_{i=2}^{p-1} s_{i}(x, y)
$$

onde $s_{i}(x, y)$ envolve comutadores que contêm $i$ letras $x$ e $p-i$ letras $y$.

Definição 2.8 Seja L uma álgebra de Lie restrita sobre um corpo $K$ de característica $p>0$. A álgebra envelopante universal restrita de $L$ é o quociente $u(L)=U(L) / J$, onde $J$ é o ideal da álgebra envelopante universal $U(L)$ gerado pelo conjunto $\left\{x^{[p]}-x^{p} \mid\right.$ $x \in L\}$.

O próximo resultado é uma versão do Teorema de Poincaré-Birkoff-Witt para álgebras envelopantes restritas. Através de uma base ordenada qualquer de uma álgebra de Lie restrita $L$, podemos obter uma base para $u(L)$.

Teorema 2.9 (PBW para álgebras envelopantes restritas [21]) Para qualquer base $\left\{x_{i} \mid i \in I\right\}$ de uma álgebra de Lie restrita L, onde I é um conjunto ordenado de indices, os monômios

$$
\left\{x_{i_{1}}^{\alpha_{1}} \cdots x_{i_{n}}^{\alpha_{n}} \mid i_{1}<\ldots<i_{n}, 0 \leq \alpha_{j} \leq p-1, n \geq 0\right\}
$$

constituem uma base para a álgebra envelopante restrita $u(L)$.

Outro conceito de fundamental importância para este trabalho é o de derivação, presente no Capítulo 5.

Definição 2.10 Seja A uma álgebra de tipo arbitrário. Uma aplicação linear $\phi \in$ End $A$ é dita uma derivação de A se satisfaz a condição

$$
\phi(a \cdot b)=\phi(a) \cdot b+a \cdot \phi(b)
$$

O espaço de todas as derivações de $A$ será denotado por Der $A$. É fácil verificar que Der $A$ é uma subálgebra da álgebra de Lie $\operatorname{End}^{(-)} A$. 
Sejam $L$ uma álgebra de Lie e $V$ um espaço vetorial sobre um corpo $K$. Um homomorfismo $\phi: L \rightarrow$ End $^{(-)} V$ é chamado uma representação da álgebra de Lie $L$. Então,

$$
\begin{aligned}
\phi([x, y]) & =[\phi(x), \phi(y)]=\phi(x) \phi(y)-\phi(y) \phi(x), \quad x, y \in L \\
\phi([x, y]) v & =\phi(x)(\phi(y) v)-\phi(y)(\phi(x) v), \quad x, y \in L, v \in V .
\end{aligned}
$$

Denotando $x \circ v=\phi(x) v, x \in L, v \in V$, obtemos a noção de módulo de Lie. Neste caso, a relação acima é reescrita como

$$
[x, y] \circ v=x \circ(y \circ v)-y \circ(x \circ v), \quad x, y \in L, v \in V .
$$

A noção de representação (ou módulo) é equivalente para a envelopante universal $U(L)[21]$.

Agora sejam $L, V$ álgebras de Lie, e $V$ um módulo de Lie sobre $L$. Queremos definir uma estrutura de álgebra de Lie para o espaço vetorial $H=L \oplus V$ fazendo

$$
[(x, v),(y, w)]=([x, y], x \circ w-y \circ v+[v, w]), \quad x, y \in L, v, w \in V .
$$

A anticomutatividade é evidente. Escrevemos por simplicidade $x=(x, 0), x \in L$, e $v=(0, v), v \in V$. Seja $J(X, Y, X)=[X,[Y, Z]]+[Y,[Z, X]]+[Z,[X, Y]]$ o polinômio de Jacobi. É claro que $J(x, y, z)=0$ para $x, y, z \in L$ e $J(v, w, u)=0$ para $v, w, u \in V$. Considerando $x, y \in L, v \in V$, temos

$$
\begin{aligned}
J(x, y, v) & =[x,[y, v]]+[y,[v, x]]+[v,[x, y]]=[x,[y, v]]-[y,[x, v]]-[[x, y], v] \\
& =x \circ(y \circ v)-y \circ(x \circ v)-[x, y] \circ v=0
\end{aligned}
$$

porque $V$ é um módulo de Lie sobre $L$. Considerando o caso restante, temos $x \in L$, $v, w \in V:$

$$
\begin{aligned}
J(x, v, w) & =[x,[v, w]]+[v,[w, x]]+[w,[x, v]] \\
& =x \circ[v, w]-[v, x \circ w]-[x \circ v, w] .
\end{aligned}
$$

O resultado obtido é nulo se, e somente se, a ação por $x \in L$ é uma derivação da álgebra de Lie $V$.

Então, sejam $L, V$ álgebras de Lie, e $V$ um módulo de Lie sobre $L$ tal que $L$ age sobre $V$ como derivações (isto é, temos um homomorfismo $\phi: L \rightarrow$ Der $V$ ). Neste caso, $L \oplus V$ tem estrutura de álgebra de Lie, denominada produto semidireto, denotado por $L \curlywedge V$. 
Todas essas noções são naturalmente generalizadas também para superálgebras de Lie.

\section{$2.2 \quad$ Álgebras graduadas}

Para introduzirmos o conceito de superálgebra de Lie, precisamos da definição de álgebra graduada.

Definição 2.11 Seja $G$ um grupo. Uma álgebra $A$ é dita G-graduada se para cada $g \in G$ existe um subespaço $A_{g}$ de $A$ de modo que:

(i) $A=\bigoplus_{g \in G} A_{g}$;

(ii) $A_{g} A_{h} \subseteq A_{g h}$ para quaisquer $g, h \in G$.

Nesse caso, a decomposição $A=\bigoplus_{g \in G} A_{g}$ é denominada G-graduação de $A$.

Dizemos que uma $G$-graduação de uma álgebra $A=\underset{g \in G}{\oplus} A_{g}$ é fina se não existem um grupo $H$ e uma graduação $A=\bigoplus_{h \in H} A_{h}$ tal que para qualquer $0 \neq A_{h}, h \in H$, existe $g \in G$ tal que $A_{h} \subset A_{g}$ e alguma dessas inclusões é própria. Em particular, se todas as componentes $A_{g}, g \in G$, são no máximo unidimensionais, obtemos uma graduação fina.

\subsection{Superálgebras de Lie}

Definição 2.12 Uma superálgebra é uma álgebra $\mathbb{Z}_{2}$-graduada $A=A_{\overline{0}} \oplus A_{\overline{1}}$.

Um elemento $a \in A_{\alpha}, \alpha \in \mathbb{Z}_{2}$, é dito homogêneo de grau $\alpha$. Nesse caso, escrevemos $\operatorname{deg} a=\alpha$. Os elementos de $A_{\overline{0}}$ são denominados pares e os de $A_{\overline{1}}$, ímpares. De agora em diante, sempre que em uma expressão aparecer o grau de algum elemento $a$, $\operatorname{deg} a$, assumiremos que tal elemento é homogêneo, e a expressão se estende aos demais elementos por linearidade.

Definição 2.13 Sejam $A$ e $B$ duas superálgebras sobre um corpo $K$. O produto tensorial de superálgebras $A \otimes B$ é a superálgebra cujo espaço é o produto tensorial dos espaços $A$ e $B$ com $\mathbb{Z}_{2}$-graduação induzida, e cujo produto é definido por

$$
\left(a_{1} \otimes b_{1}\right) \cdot\left(a_{2} \otimes b_{2}\right)=(-1)^{\operatorname{deg} b_{1} \cdot \operatorname{deg} a_{2}} a_{1} a_{2} \otimes b_{1} b_{2}, \quad a_{i} \in A, b_{i} \in B
$$


Definição 2.14 Uma superálgebra associativa é uma álgebra associativa $\mathbb{Z}_{2}$-graduada $A=A_{\overline{0}} \oplus A_{\overline{1}}$.

Definição 2.15 Uma superálgebra de Lie é uma álgebra $\mathbb{Z}_{2}$-graduada $L=L_{\overline{0}} \oplus L_{\overline{1}}$ com um produto [, ] (denominado supercolchete de Lie) satisfazendo os seguintes axiomas:

(i) $[x, y]=-(-1)^{\operatorname{deg} x \cdot \operatorname{deg} y}[y, x]$ (superanticomutatividade);

(ii) $[x,[y, z]]=[[x, y], z]+(-1)^{\operatorname{deg} x \cdot \operatorname{deg} y}[y,[x, z]]$ (superidentidade de Jacobi).

Em particular, essas condições implicam que $L_{\overline{0}}$ é uma álgebra de Lie e $L_{\overline{1}}$ é um módulo sobre $L_{\overline{0}}$. Neste trabalho, supercolchetes de grandes comprimentos serão normados à direita: $[x, y, z]=[x,[y, z]]$. Para cada $x \in L$, definimos o operador adjunto ad $x: L \rightarrow$ $L$ por ad $x(y)=[x, y]$. Dizemos que o elemento $x \in L$ é ad-nilpotente se $(\operatorname{ad} x)^{n}=0$ para algum inteiro positivo $n$.

Seja $A=A_{\overline{0}} \oplus A_{\overline{1}}$ uma superálgebra associativa. Podemos definir no mesmo espaço vetorial $A$ um supercomutador

$$
[x, y]=x y-(-1)^{\operatorname{deg} x \cdot \operatorname{deg} y} y x, \quad x, y \in A .
$$

Esse produto faz de $A$ uma superálgebra de Lie, a qual denotamos por $A^{(-)}$. Por outro lado, a qualquer superálgebra de Lie $L$ podemos associar uma álgebra associativa, como veremos a seguir.

Definição 2.16 Seja $L=L_{\overline{0}} \oplus L_{\overline{1}}$ uma superálgebra de Lie sobre um corpo $K$. Uma álgebra envelopante universal de L é um par $(U, i)$, onde $U$ é uma álgebra associativa unitária sobre $K$ e $i: L \rightarrow U$ é uma aplicação linear que satisfaz a condição

$$
i([x, y])=i(x) i(y)-(-1)^{\operatorname{deg} x \cdot \operatorname{deg} y} i(y) i(x)
$$

com a seguinte propriedade universal: se A é uma álgebra associativa unitária qualquer sobre $K$ e $\sigma: L \rightarrow A$ é uma transformação linear satisfazendo (2.2), então existe um único homomorfismo de álgebras associativas $\phi: U \rightarrow A$ tal que $\phi\left(1_{U}\right)=1_{A}$ e $\phi \circ i=\sigma$.

Observe que, na definição acima, a álgebra $A$ não precisa ser $\mathbb{Z}_{2}$-graduada.

Para qualquer superálgebra de Lie $L$, existe um par $(U, i)$ como na Definição 2.16. E esse par é único, a menos de isomorfismos. Podemos obter uma álgebra envelopante 
para $L$ como segue. Seja $T(L)$ a álgebra tensorial de $L$, e $J$ o ideal de $T(L)$ gerado pelos elementos

$$
x \otimes y-y \otimes x-(-1)^{\operatorname{deg} x \cdot \operatorname{deg} y}[x, y], \quad x, y \in L .
$$

Tomando $U(L)=T(L) / J$ e considerando $i: L \rightarrow U(L)$ a restrição do homomorfismo canônico $\pi: T(L) \rightarrow U(L)$ a $L$, então o par $(U(L), i)$ é uma álgebra envelopante universal da álgebra de Lie $L$. Para mais detalhes, veja [20].

O teorema abaixo é uma versão de PBW para superálgebras de Lie $L=L_{\overline{0}} \oplus L_{\overline{1}}$. Se conhecemos bases ordenadas para as componentes homogêneas $L_{\overline{0}}$ e $L_{\overline{1}}$, podemos obter uma base para a álgebra envelopante $U(L)$.

Teorema 2.17 (Poincaré-Birkhoff-Witt para superálgebras de Lie) Seja $X_{0}$ uma base de $L_{\overline{0}}$ e $X_{1}$ uma base de $L_{\overline{1}}$ sobre $K$. Se $\leq$ é uma ordem total em $X=X_{0} \cup X_{1}$, então o conjunto de todos os monômios da forma

$$
x_{1} x_{2} \cdots x_{n}, \quad n \geq 0,
$$

com $x_{i} \in X, x_{i} \leq x_{i+1}$, e $x_{i} \neq x_{i+1}$ se $x_{i} \in X_{1}$, é uma base para $U(L)$ sobre $K$.

No caso de características pequenas char $K=2$, 3 , é necessário acrescentar alguns axiomas aos apresentados na definição de superálgebra de Lie. Substituindo $x=y=$ $z \in L_{\overline{1}}$ na superidentidade de Jacobi e usando a superanticomutatividade, obtemos $3[z,[z, z]]=0, z \in L_{\overline{1}}$. Então, se char $K \neq 3$, temos a identidade: $[z,[z, z]]=0$ para todo $z \in L_{\overline{1}}$. Para característica 3 , adicionamos essa identidade aos axiomas de superálgebra de Lie.

- $(\operatorname{char} K=3)[z,[z, z]]=0, z \in L_{\overline{1}}$.

Substituindo $x=y \in L_{\overline{1}}$ na superidentidade de Jacobi, obtemos $2(\operatorname{ad} x)^{2} z=[[x, x], z]$. Se char $K \neq 2$, vale a identidade

$$
(\operatorname{ad} x)^{2} z=\frac{1}{2}[[x, x], z], \quad x \in L_{\overline{1}}, z \in L
$$

Neste trabalho, lidamos com superálgebras de Lie da forma $A^{(-)}$, onde $A$ é uma superálgebra associativa. Nesse caso, temos quadrados $[x, x]=2 x^{2}, x \in A_{\overline{1}}^{(-)}$. Dessa 
forma, a identidade acima tem a seguinte forma (pode-se verificar diretamente para característica 2):

$$
(\operatorname{ad} x)^{2} z=\left[x^{2}, z\right], \quad x \in A_{\overline{1}}^{(-)}, z \in A^{(-)} .
$$

Se char $K=2$, adicionamos mais estes axiomas para superálgebras de Lie:

- $[x, x]=0$ para todo $x \in L_{\overline{0}}$;

- existe uma aplicação quadrática (quadrado formal)

$$
\begin{aligned}
(*)^{[2]}: L_{\overline{1}} & \longrightarrow L_{\overline{0}} \\
x & \longmapsto x^{[2]},
\end{aligned}
$$

que satisfaz

$$
(\lambda x)^{[2]}=\lambda^{2} x^{[2]}, \quad(x+y)^{[2]}=x^{[2]}+[x, y]+y^{[2]} ; \quad x, y \in L_{\overline{1}}, \lambda \in K
$$

- uma identidade análoga a (2.3) é válida:

$$
(\operatorname{ad} x)^{2} z=\left[x^{[2]}, z\right], \quad x \in L_{\overline{1}}, z \in L
$$

No caso $p=2$, para obtermos a álgebra envelopante universal, além de tomarmos o quociente de $T(L)$ por $J=\left(x \otimes y-y \otimes x-(-1)^{\operatorname{deg} x \cdot \operatorname{deg} y}[x, y] \mid x, y \in L\right)$, precisamos tomar também o quociente pelo ideal $\left(y \otimes y-y^{[2]} \mid y \in L_{\overline{1}}\right)$. Uma superálgebra de Lie sobre um corpo $K$ de característica 2 é, grosso modo, o mesmo que uma álgebra de Lie $\mathbb{Z}_{2}$-graduada com uma aplicação quadrática $L_{\overline{1}} \rightarrow L_{\overline{0}}$ similar a uma $p$-aplicação (veja abaixo). Em todos os casos, a aplicação quadrática coincide com o quadrado ordinário na álgebra envelopante $U(L)$ e será denotada simplesmente por $x^{2}, x \in L_{\overline{1}}$.

\subsection{Crescimento de álgebras}

Introduziremos agora o conceito de crescimento. Seja $A$ uma álgebra associativa (ou de Lie) gerada por um conjunto $X$. Denotemos por $A^{(X, n)}$ o subespaço de $A$ gerado por todos os monômios em $X$ de comprimento menor ou igual a $n, n \geq 0$. Temos

$$
A^{(X, n)}=\left\langle x_{1} \cdots x_{s} \mid x_{i} \in X, s \leq n\right\rangle_{K}
$$

No caso específico de uma superálgebra de Lie de char $K=2$, também consideramos os quadrados formais dos monômios ímpares de comprimento no máximo $n / 2$. Se $A$ é 
uma álgebra de Lie restrita, fazemos $A^{(X, n)}=\left\langle\left[x_{1}, \ldots x_{s}\right]^{p^{k}} \mid x_{i} \in X, s p^{k} \leq n\right\rangle_{K}[31]$. A definição de $A^{(X, n)}$ é análoga para superálgebras de Lie restritas. Em qualquer situação, obtemos uma função de crescimento:

$$
\gamma_{A}(n)=\gamma_{A}(X, n)=\operatorname{dim}_{K} A^{(X, n)}, \quad n \geq 0
$$

Sejam $f, g: \mathbb{N} \rightarrow \mathbb{R}^{+}$duas funções monótonas não decrescentes com valores positivos. Escrevemos $f(n) \preccurlyeq g(n)$ se, e somente se, existem duas constantes $N, C>0$ tais que $f(n) \leq g(C n)$ para todo $n \geq N$. A equivalência $f(n) \sim g(n)$ ocorre se, e somente se, $f(n) \preccurlyeq g(n)$ e $g(n) \preccurlyeq f(n)$. Conjuntos geradores distintos produzem funções de crescimento equivalentes [23, Ch. 1, Lemma 1.1].

Sabe-se que o crescimento exponencial é o maior crescimento possível para álgebras associativas e álgebras de Lie finitamente geradas. Uma função de crescimento $\gamma_{A}(n)$ é comparada com funções polinomiais $n^{k}, k \in \mathbb{R}^{+}$, ao computar-se a dimensão de Gelfand-Kirillov [23], definida a seguir.

Definição 2.18 Seja A uma (super)álgebra associativa (ou de Lie). A dimensão de Gelfand-Kirillov superior (ou, simplesmente, dimensão de Gelfand-Kirillov) de $A$ é dada por

$$
\operatorname{GKdim} A=\inf \left\{\alpha>0 \mid \gamma_{A}(n) \preccurlyeq n^{\alpha}\right\} .
$$

$A$ dimensão de Gelfand-Kirilov inferior de $A$ é dada por

$$
\underline{\operatorname{GKdim}} A=\sup \left\{\alpha>0 \mid \gamma_{A}(n) \succcurlyeq n^{\alpha}\right\}
$$

Não é difícil verificar que GKdim $A=\varlimsup_{n \rightarrow \infty} \frac{\ln \gamma_{A}(n)}{\ln n}$ e que $\underline{\operatorname{GKdim}} A=\varliminf_{n \rightarrow \infty} \frac{\ln \gamma_{A}(n)}{\ln n}$. Assuma que os geradores $X=\left\{x_{1}, \ldots, x_{k}\right\}$ possuem pesos positivos $\mathrm{wt}\left(x_{i}\right)=\lambda_{i}$, $i=1, \ldots, k$. Definimos uma função de crescimento ponderada:

$$
\tilde{\gamma}_{A}(n)=\operatorname{dim}_{K}\left\langle x_{i_{1}} \cdots x_{i_{m}} \mid \operatorname{wt}\left(x_{i_{1}}\right)+\cdots+\operatorname{wt}\left(x_{i_{m}}\right) \leq n, x_{i_{j}} \in X\right\rangle_{K}, \quad n \geq 0 .
$$

Se $C_{1}=\min \left\{\lambda_{i} \mid i=1, \ldots, k\right\}$ e $C_{2}=\max \left\{\lambda_{i} \mid i=1, \ldots, k\right\}$, então $\tilde{\gamma}_{A}\left(C_{1} n\right) \leq$ $\gamma_{A}(n) \leq \tilde{\gamma}_{A}\left(C_{2} n\right)$ para $n \geq 1$. Assim, obtemos uma função de crescimento equivalente $\tilde{\gamma}_{A}(n) \sim \gamma_{A}(n)$. Portanto, podemos utilizar a função de crescimento ponderada $\tilde{\gamma}_{A}(n)$ para computar as dimensões de Gelfand-Kirillov.

Seja $L$ uma (super)álgebra de Lie e $X \subset L$. Denotamos por $\operatorname{Lie}(X)$ a subálgebra de $L$ gerada por $X$, incluindo a aplicação quadrática no caso char $K=2$. Se $L$ é 
uma (super)álgebra de Lie restrita, denotamos por $\operatorname{Lie}_{p}(X)$ a subálgebra restrita de $L$ gerada por $X$. Semelhantemente, se $X$ é um subconjunto de uma álgebra associativa $A$, denotamos por $\operatorname{Alg}(X) \subset A$ a subálgebra associativa gerada por $X$.

\section{5 Álgebra de Lie das derivações especiais}

O conceito trazido nesta seção será útil para a construção feita no Capítulo 5. Suponhamos que char $K=p>0$. Usando a notação $I=\{0,1,2, \ldots\}$ e $\mathbb{N}_{p}=\{0,1, \ldots, p-1\}$, consideremos a álgebra polinomial truncada

$$
\Delta=K\left[x_{i}, i \in I \mid x_{i}^{p}=0, i \in I\right]
$$

Seja $\mathbb{N}_{p}^{I}=\left\{\alpha: I \rightarrow \mathbb{N}_{p}\right\}$ o conjunto de funções com um número finito de valores não nulos. Para $\alpha \in \mathbb{N}_{p}^{I}$, denotemos $|\alpha|=\sum_{i \in I} \alpha_{i}$ e $\mathbf{x}^{\alpha}=\prod_{i \in I} x_{i}^{\alpha_{i}} \in \Delta$. O conjunto $\left\{\mathbf{x}^{\alpha} \mid \alpha \in \mathbb{N}_{p}^{I}\right\}$ é claramente uma base de $\Delta$. Consideremos o ideal $\Delta^{+}$gerado por todos os elementos $\mathbf{x}^{\alpha}, \alpha \in \mathbb{N}_{p}^{I},|\alpha|>0$. Denotemos por $\partial_{i}=\frac{\partial}{\partial x_{i}}, i \in I$, as derivações parciais de $\Delta$.

Introduzimos a denominada álgebra de Lie das derivações especiais de $\Delta$ [41], [42], [35]:

$$
\mathbf{W}(\Delta)=\left\{\sum_{\alpha \in \mathbb{N}_{p}^{I}} \mathbf{x}^{\alpha} \sum_{j=1}^{|\alpha|} \lambda_{\alpha, i_{j}} \frac{\partial}{\partial x_{i_{j}}} \mid \lambda_{\alpha, i_{j}} \in K, i_{j} \in I\right\}
$$

É essencial que a soma para cada $\mathbf{x}^{\alpha}, \alpha \in \mathbb{N}_{p}^{I}$, seja finita.

Lema 2.19 ([36]) Para números complexos arbitrários $a_{i} \in \mathbb{C}, i \in \mathbb{N}$, existem graduações nas álgebras $\Delta, \mathbf{W}(\Delta)$ tais que $\operatorname{wt}\left(x_{i}\right)=-a_{i}$, wt $\left(\partial_{i}\right)=a_{i}$.

\subsection{Superálgebra de Lie das superderivações}

Seja $V=V_{\overline{0}} \oplus V_{\overline{1}}$ um espaço vetorial $\mathbb{Z}_{2}$-graduado. Denotemos por End $V$ a álgebra associativa formada por todos os endomorfismos do espaço $V$. Para $\alpha \in \mathbb{Z}_{2}$, consideremos os endomorfismos de grau $\alpha$ :

$$
\operatorname{End}_{\alpha} V=\left\{\phi \in \text { End } V \mid \phi\left(V_{\beta}\right) \subset V_{\alpha+\beta}, \beta \in \mathbb{Z}_{2}\right\}
$$


Os elementos de $\operatorname{End}_{\overline{0}} V$ são denominados endomorfismos pares e os de $\operatorname{End}_{\overline{1}} V$, endomorfismos impares. Consideremos um endomorfismo $\phi \in$ End $V$ e um elemento $u \in V$ quaisquer. Uma vez que $V=V_{\overline{0}} \oplus V_{\overline{1}}$, podemos escrever de forma única $v=v_{0}+v_{1}$, onde $v_{0} \in V_{\overline{0}}$ e $v_{1} \in V_{\overline{1}}$. Também escrevemos de modo único $\phi\left(v_{0}\right)=u_{0}+u_{1}$ e $\phi\left(v_{1}\right)=w_{0}+w_{1}$, com $u_{0}, w_{0} \in V_{\overline{0}}$ e $u_{1}, w_{1} \in V_{\overline{1}}$. Definindo $\phi_{0}(v):=u_{0}+w_{1}$ e $\phi_{1}(v):=u_{1}+w_{0}$, obtemos um endomorfismo par $\phi_{0} \in \operatorname{End}_{\overline{0}} V$ e um endomorfismo ímpar $\phi_{1} \in \operatorname{End}_{\overline{1}} V$ tais que $\phi=\phi_{0}+\phi_{1}$. Através dessa decomposição, obtemos uma soma direta

$$
\text { End } V=\operatorname{End}_{\overline{0}} V \oplus \operatorname{End}_{\overline{1}} V
$$

com a qual End $V$ possui uma estrutura de superálgebra associativa. Dessa forma, End $^{(-)} V$ é uma superálgebra de Lie, denominada superálgebra linear geral, a qual é denotada por $g l(V)$.

Outro conceito muito importante neste trabalho é o de superderivação, presente no Capítulo 4.

Definição 2.20 Seja $A=A_{\overline{0}} \oplus A_{\overline{1}}$ uma álgebra $\mathbb{Z}_{2}$-graduada de tipo arbitrário. Uma aplicação linear $\phi \in \operatorname{End}_{\beta} A, \beta \in \mathbb{Z}_{2}$, é uma superderivação de grau $\beta$ se satisfaz a condição

$$
\phi(a \cdot b)=\phi(a) \cdot b+(-1)^{\beta \cdot \operatorname{deg} a} a \cdot \phi(b), \quad a, b \in A .
$$

O espaço de todas as superderivações de $A$ de grau $\alpha \in \mathbb{Z}_{2}$ será denotado por $\operatorname{Der}_{\alpha} A \subset$ $\operatorname{End}_{\alpha} A$. É fácil verificar que Der $A=\operatorname{Der}_{\overline{0}} A \oplus \operatorname{Der}_{\overline{1}} A$ é uma subálgebra da superálgebra de Lie $\operatorname{End}^{(-)} A$.

Definição 2.21 Uma superálgebra de Lie restrita $L=L_{\overline{0}} \oplus L_{\overline{1}}$ é uma superálgebra de Lie tal que a componente par $L_{\overline{0}}$ é uma álgebra de Lie restrita e $L_{\overline{1}}$ é um módulo sobre $L_{\overline{0}}$ restrito, ou seja, $\operatorname{ad}\left(x^{[p]}\right) y=(\operatorname{ad} x)^{p} y$ para quaisquer $x \in L_{\overline{0}}, y \in L_{\overline{1}}$.

Observamos que, para char $K=2$, as superálgebras de Lie restritas e as álgebras de Lie restritas $\mathbb{Z}_{2}$-graduadas são exatamente os mesmos objetos. Se $L=L_{\overline{0}} \oplus L_{\overline{1}}$ é uma superálgebra de Lie restrita, estendemos a 2-aplicação para toda a álgebra fazendo $(x+y)^{[2]}=x^{[2]}+y^{[2]}+[x, y], x \in L_{\overline{0}}, y \in L_{\overline{1}}$.

Seja $\Lambda_{n}=\Lambda\left(x_{1}, \ldots, x_{n}\right)$ a álgebra de Grassmann em $n$ variáveis. Definindo deg $x_{i}=1$, $i=1, \ldots, n$, temos a superálgebra associativa $\Lambda_{n}=\Lambda_{\overline{0}} \oplus \Lambda_{\overline{1}}$, denominada superálgebra de Grassmann. Como o supercolchete em $\Lambda_{n}^{(-)}$é trivial, ele é denominado supercomutativo. As relações $\partial_{x_{i}}\left(x_{j}\right)=\delta_{i j}$, para $i, j \in\{1, \ldots, n\}$, definem superderivações 
$\partial_{x_{i}} \in \operatorname{Der} \Lambda_{n}$. Toda superderivação $D \in \operatorname{Der} \Lambda_{n}$ pode ser escrita unicamente na forma $[24]$

$$
D=\sum_{i=1}^{n} P_{i} \partial_{x_{i}}, \quad P_{i} \in \Lambda_{n}
$$

Se char $K=0$, obtemos uma superálgebra de Lie de Cartan do tipo $\mathbf{W}_{n}$ simples de dimensão finita [24]. Para mais informações a respeito da teoria de superálgebras de Lie, veja $[24,47,2]$.

Seja $I$ um conjunto bem ordenado de cardinalidade arbitrária. Consideremos $\mathbb{Z}_{2}=$ $\{0,1\}$ e seja $\mathbb{Z}_{2}^{I}=\left\{\alpha: I \rightarrow \mathbb{Z}_{2}\right\}$ um conjunto de funções com um número finito de valores não nulos. Suponha que $\alpha \in \mathbb{Z}_{2}^{I}$ assume valores não nulos $\left\{i_{1}, \ldots, i_{t}\right\} \subset I$, onde $i_{1}<\cdots<i_{t}$. Façamos $\mathbf{x}^{\alpha}=x_{i_{1}} x_{i_{2}} \cdots x_{i_{t}}$ e $|\alpha|=t$. O conjunto $\left\{\mathbf{x}^{\alpha} \mid \alpha \in \mathbb{Z}_{2}^{I}\right\}$ é uma base da álgebra de Grassmann $\Lambda_{I}=\Lambda\left(x_{i} \mid i \in I\right)$, que é uma superálgebra associativa $\Lambda_{I}=\Lambda_{\overline{0}} \oplus \Lambda_{\overline{1}}$, onde todo elemento $x_{i} \operatorname{com} i \in I$ é ímpar. Denotemos por $\partial_{x_{i}}, i \in I$, as superderivações de $\Lambda_{I}$ determinadas pelos valores $\partial_{x_{i}}\left(x_{j}\right)=\delta_{i j}, i, j \in I$. Consideremos o espaço vetorial de todas as somas formais

$$
\mathbf{W}\left(\Lambda_{I}\right)=\left\{\sum_{\alpha \in \mathbb{Z}_{2}^{I}} \mathbf{x}^{\alpha} \sum_{j=1}^{|\alpha|} \lambda_{\alpha, i_{j}} \partial_{x_{i_{j}}} \mid \lambda_{\alpha, i_{j}} \in K, i_{j} \in I\right\} .
$$

É essencial que a soma para cada $\mathbf{x}^{\alpha}, \alpha \in \mathbb{Z}_{2}^{I}$, seja finita. Essa construção é semelhante à álgebra de Lie das derivações especiais. Para mais informações, veja [41, 42, 35]. Verifica-se de modo similar que o produto entre elementos de $\mathbf{W}\left(\Lambda_{I}\right)$ está bem definido e $\mathbf{W}\left(\Lambda_{I}\right)$ age sobre $\Lambda_{I}$ por superderivações. No Exemplo 1, a superálgebra $\mathbf{R}$ definida será construída como subálgebra de $\mathbf{W}\left(\Lambda_{I}\right) \subset \operatorname{Der} \Lambda_{I}$. 


\section{Capítulo 3}

\section{Resultados principais}

Neste capítulo, exibiremos um exemplo de superálgebra de Lie e um exemplo de álgebra de Lie restrita satisfazendo algumas propriedades. Listaremos aqui os principais resultados obtidos acerca desses exemplos, a serem detalhados no Capítulo 4 e no Capítulo 5.

\subsection{A superálgebra de Lie $\mathrm{R}$}

Dando continuidade ao que foi feito por Petrogradsky [34], construímos outro exemplo de superálgebra de Lie análoga aos grupos de Grigorchuk e Gupta-Sidki. Para um corpo qualquer, construímos uma superálgebra de Lie fractal $\mathbf{R}$ em que os elementos homogêneos na $\mathbb{Z}_{2}$-graduação são ad-nilpotentes. O diferencial do nosso exemplo está no fato de que $\mathbf{R}$ tem crescimento linear, além de apresentar uma construção elegante, com base monomial evidente e elementos de fáceis computações.

A superálgebra $\mathbf{R}$ tem uma $\mathbb{Z}^{2}$-graduação natural cujas componentes homogêneas são no máximo unidimensionais, e os elementos homogêneos da $\mathbb{Z}_{2}$-graduação $\mathbf{R}=\mathbf{R}_{\overline{0}} \oplus \mathbf{R}_{\overline{1}}$ são ad-nilpotentes. Trata-se, então, de um contraexemplo para uma possível extensão do Teorema 1.1 para o campo das superálgebras de Lie.

Neste exemplo, nosso objetivo é estudar a seguinte superálgebra de Lie finitamente gerada. Consideramos uma superálgebra de Grassmann $\Lambda$ em infinitas variáveis. Escrevemos nossos elementos como somas infinitas pertencentes à superálgebra de Lie $\mathbf{W}(\Lambda) \subset \operatorname{Der} \Lambda$. Consideramos que existe uma imersão natural da álgebra de Grassmann $\Lambda$ na álgebra End $\Lambda$, associando cada elemento de $\Lambda$ à multiplicação à esquerda por esse elemento. 
Seja $\Lambda=\Lambda\left[x_{i} \mid i \geq 0\right]$ e considere os seguintes elementos ímpares em $\mathbf{W}(\Lambda)$ :

$$
v_{i}=\partial_{x_{i}}+x_{i} x_{i+1}\left(\partial_{x_{i+2}}+x_{i+2} x_{i+3}\left(\partial_{x_{i+4}}+x_{i+4} x_{i+5}\left(\partial_{x_{i+6}}+\cdots\right)\right)\right), \quad i \geq 0
$$

Definimos uma superálgebra de Lie $\mathbf{R}=\operatorname{Lie}\left(v_{0}, v_{1}\right) \subset \mathbf{W}(\Lambda)$ e sua envoltória associativa $\mathbf{A}=\operatorname{Alg}\left(v_{0}, v_{1}\right) \subset$ End $\Lambda$. No caso em que char $K=2$, assumimos que a aplicação quadrática sobre os elementos ímpares coincide com o quadrado do endomorfismo correspondente (multiplicação à esquerda) em End $\Lambda$.

Dizemos que $\left\{v_{i} \mid i \geq 0\right\}$ são os elementos pivô. Estabelecemos as seguintes propriedades principais de $\mathbf{R}=\operatorname{Lie}\left(v_{0}, v_{1}\right)$ e de sua envoltória associativa $\mathbf{A}=\operatorname{Alg}\left(v_{0}, v_{1}\right)$.

(i) No início do Capítulo 4, obtemos relações básicas entre elementos de $\mathbf{R}$ e mostramos que $\mathbf{R}$ é autossimilar.

(ii) Se char $K \neq 2, \mathbf{R}$ tem uma base composta por monômios standard de dois tipos (Teorema 4.5). No caso em que char $K=2$, obtemos uma base de $\mathbf{R}$ formada por monômios do primeiro tipo e quadrados de elementos pivô (Corolário 4.6). Então, temos bases nítidas.

(iii) No caso em que char $K=p>0$, também consideramos uma superálgebra de Lie restrita $\operatorname{Lie}_{p}\left(v_{0}, v_{1}\right)$, que para char $K=2$ coincide com a álgebra de Lie restrita também denotada por $\operatorname{Lie}_{p}\left(v_{0}, v_{1}\right)$, e a base coincide com a base de $\mathbf{R}$.

(iv) Introduzimos duas funções peso wt, swt, que são aditivas em produtos de monômios (Seção 4.3).

(v) Obtemos uma $\mathbb{Z}^{2}$-graduação $\mathbf{R}=\underset{n_{1}, n_{2} \geq 0}{\oplus} \mathbf{R}_{n_{1} n_{2}}$ pelo multigrau em relação aos geradores $\left\{v_{0}, v_{1}\right\}$ (Lema 4.9). Mostramos que essa $\mathbb{Z}^{2}$-graduação é fina, isto é, $\operatorname{dim} \mathbf{R}_{n_{1} n_{2}} \leq 1, n_{1}, n_{2} \geq 0$. Introduzimos dois sistemas de coordenadas no plano: as coordenadas multigrau $\left(X_{1}, X_{2}\right)$ e as coordenadas peso $\left(Z_{1}, Z_{2}\right)$ (Seção 4.4).

(vi) Encontramos limitações para os pesos dos monômios de $\mathbf{R}$ e $\mathbf{A}$ (Seção 4.5). Na representação geométrica, os monômios de $\mathbf{R}$ estão em uma região do plano limitada por duas curvas logarítmicas (Teorema 4.18). Para a envoltória associativa A, valem limitações similares. A estrutura das componentes homogêneas está bem clara na Figura 4.5, página 50. 
(vii) $G K \operatorname{dim} \mathbf{R}=\underline{G K \operatorname{dim}} \mathbf{R}=1$ (Teorema 4.20). Se char $K \neq 2$, a função de crescimento ponderada de $\mathbf{R}$ é linear. A função de crescimento usual satisfaz

$$
\begin{aligned}
& \lim _{m \rightarrow \infty} \frac{\gamma(m)}{m}=3, \quad \text { no caso } p \neq 2 \\
& \lim _{m \rightarrow \infty} \frac{\gamma(m)}{m}=\frac{3}{2}, \quad \text { no caso } p=2
\end{aligned}
$$

A superálgebra Q construída em [34] foi "pequena", $\operatorname{com} \operatorname{GKdim} \mathbf{Q} \approx 1.89$. Entretanto, o nosso exemplo $\mathbf{R}$ é ainda "menor", com GKdim $\mathbf{R}=1$ e crescimento linear. Essa é a virtude do nosso novo exemplo.

(viii) $\mathrm{GK \operatorname {dim }} \mathbf{A}=\underline{\mathrm{GKdim}} \mathbf{A}=2$, o crescimento de $\mathbf{A}$ é quadrático (Teorema 4.26).

(ix) Na seção 4.7, descrevemos funções geradoras para $\mathbf{R}$.

(x) $\mathbf{R}=\mathbf{R}_{\overline{0}} \oplus \mathbf{R}_{\overline{1}}$ é uma superálgebra de Lie $\mathbb{Z}_{2}$-graduada nil (Teorema 4.32). Obtemos uma decomposição triangular em soma direta de três subálgebras localmente nilpotentes $\mathbf{R}=\mathbf{R}_{+} \oplus \mathbf{R}_{0} \oplus \mathbf{R}_{-}$(Teorema 4.34).

(xi) R é uma álgebra just infinite (Seção 4.9). Destacamos que, para a superálgebra [34], isso ainda não é conhecido.

(xii) A é não nil nos casos $p=0$ e $p=2$ (Lema 4.31). Para o exemplo [34], ainda não se sabe se tal álgebra $\mathbf{A}$ é nil ou não.

\subsection{A álgebra de Lie restrita $\mathrm{R}$}

No Capítulo 5, construímos uma álgebra de Lie restrita $\mathbf{R}$ sobre um corpo $K$ de característica $p>0$. Essa álgebra, assim como o exemplo de superálgebra construído no Capítulo 4, é autossimilar e tem crescimento linear. Além disso, $\mathbf{R}$ tem uma $\mathbb{Z}^{2}$ graduação cujas componentes homogêneas são no máximo unidimensionais. Contudo, a álgebra de Lie restrita $\mathbf{R}$ não possui uma $p$-aplicação nil.

Para este exemplo, vamos considerar char $K=p>0$. Seja $\Delta=K\left[x_{i} \mid i \geq 0\right] /\left(x_{i}^{p}\right)$ uma álgebra de polinômios truncada. Considere os seguintes elementos em $\mathbf{W}(\Delta)$ :

$$
v_{i}=\partial_{x_{i}}+x_{i}^{p-1} x_{i+1}^{p-1}\left(\partial_{x_{i+2}}+x_{i+2}^{p-1} x_{i+3}^{p-1}\left(\partial_{x_{i+4}}+x_{i+4}^{p-1} x_{i+5}^{p-1}\left(\partial_{x_{i+6}}+\cdots\right)\right)\right), \quad i \geq 0 .
$$

Definimos a álgebra de Lie $\mathbf{L}=\operatorname{Lie}\left(v_{0}, v_{1}\right) \subset \mathbf{W}(\Delta)$, a álgebra de Lie restrita $\mathbf{R}=$ $\operatorname{Lie}_{p}\left(v_{0}, v_{1}\right)$ e sua envoltória associativa $\mathbf{A}=\operatorname{Alg}(\mathbf{R}) \subset$ End $\Delta$. 
Os elementos $\left\{v_{i} \mid i \geq 0\right\}$ também são chamados de elementos pivô. Estabelecemos as seguintes propriedades principais de $\mathbf{R}=\operatorname{Lie}_{p}\left(v_{0}, v_{1}\right)$ e de sua envoltória associativa $\mathbf{A}=\operatorname{Alg}(\mathbf{R})$.

(i) No início do Capítulo 5, obtemos relações básicas entre elementos de $\mathbf{R}$ e mostramos que $\mathbf{R}$ é autossimilar.

(ii) L tem uma base composta por monômios standard do primeiro tipo (Teorema 5.6), ao passo que $\mathbf{R}$ possui uma base formada por monômios de dois tipos (Corolário 5.7). A base obtida é bem clara.

(iii) Introduzimos duas funções peso wt, swt, que são aditivas em produtos de monômios (Seção 5.3).

(iv) $\mathbf{R}$ é $\mathbb{Z}^{2}$-graduada pelo multigrau em relação aos geradores $\left\{v_{0}, v_{1}\right\}$ (Lema 5.10). Introduzimos dois sistemas de coordenadas no plano: as coordenadas multigrau $\left(X_{1}, X_{2}\right)$ e as coordenadas peso $\left(Z_{1}, Z_{2}\right)$ (Seção 5.4$)$.

(v) Encontramos limitações para os pesos dos monômios de $\mathbf{R}$ e $\mathbf{A}$ (Seção 5.5). Na representação geométrica, os monômios de $\mathbf{R}$ estão em uma região do plano limitada por duas curvas logarítmicas (Teorema 5.20). Para a envoltória associativa A, valem limitações similares.

(vi) $\operatorname{GKdim} \mathbf{R}=\underline{\operatorname{GKdim}} \mathbf{R}=1$ (Teorema 5.22).

(vii) GKdim $\mathbf{A}=\underline{G K \operatorname{dim}} \mathbf{A}=2$ (Teorema 5.28), o crescimento de $\mathbf{A}$ é quadrático.

(viii) As componentes homogêneas da $\mathbb{Z}^{2}$-graduação de $\mathbf{R}$ são no máximo unidimensionais (Teorema 5.31). Obtemos uma decomposição triangular em soma direta de três subálgebras localmente nilpotentes $\mathbf{R}=\mathbf{R}_{+} \oplus \mathbf{R}_{0} \oplus \mathbf{R}_{-}$(Teorema 5.33).

(ix) A p-aplicação definida em $\mathbf{R}$ não é nil (Lema 5.32) e a álgebra associativa A é não nil. Para o exemplo construído em [40], ainda não é conhecido se A é nil ou não.

(x) $\mathbf{R}$ é uma álgebra just infinite (Seção 5.8). Essa propriedade também não é conhecida para o exemplo [40]. 


\section{Capítulo 4}

\section{A superálgebra de Lie $\mathrm{R}$}

Neste capítulo, estudaremos as superálgebras de Lie do Exemplo 1. Consideremos a álgebra de Grassmann $\Lambda=\Lambda\left[x_{i} \mid i \geq 0\right]$. Em $\mathbf{W}(\Lambda)$, destacamos os seguintes elementos ímpares, a quem chamamos de elementos pivô:

$$
v_{i}=\partial_{x_{i}}+x_{i} x_{i+1}\left(\partial_{x_{i+2}}+x_{i+2} x_{i+3}\left(\partial_{x_{i+4}}+x_{i+4} x_{i+5}\left(\partial_{x_{i+6}}+\cdots\right)\right)\right), \quad i \geq 0 .
$$

Observe que os elementos $v_{i}$ podem ser escritos recursivamente na forma

$$
v_{i}=\partial_{x_{i}}+x_{i} x_{i+1} v_{i+2}, \quad i \geq 0 .
$$

Definimos a superálgebra de Lie $\mathbf{R}=\operatorname{Lie}\left(v_{0}, v_{1}\right)=\mathbf{R}_{\overline{0}} \oplus \mathbf{R}_{\overline{1}} \subset \mathbf{W}(\Lambda) \subset \operatorname{Der} \Lambda$ e sua envoltória associativa $\mathbf{A}=\operatorname{Alg}\left(v_{0}, v_{1}\right) \subset \operatorname{End} \Lambda$.

\subsection{Relações principais}

Para cada $i \geq 0$, definimos o endomorfismo $l_{x_{i}} \in$ End $\Lambda$ por $l_{x_{i}}(a)=x_{i} \cdot a$. Dessa forma, cada letra de Grassmann $x_{i}(i \geq 0)$ pode ser identificada com $l_{x_{i}}$ e, portanto, pode ser vista com um endomorfismo da álgebra associativa $\Lambda$. As letras de Grassmann e as superderivações $\left\{x_{i}, \partial_{x_{i}} \mid i \geq 0\right\}$ são claramente elementos ímpares de End $\Lambda$. Valem as relações anticomutativas abaixo:

$$
\begin{gathered}
x_{i} x_{j}=-x_{j} x_{i}, \quad \partial_{x_{i}} \partial_{x_{j}}=-\partial_{x_{j}} \partial_{x_{i}}, \quad \partial_{x_{i}} x_{j}=-x_{j} \partial_{x_{i}}, \quad i \neq j ; \\
x_{i}{ }^{2}=0, \quad\left(\partial_{x_{i}}\right)^{2}=0, \quad i \geq 0 .
\end{gathered}
$$


Também é válida a seguinte relação não trivial:

$$
\partial_{x_{i}} x_{i}+x_{i} \partial_{x_{i}}=1, \quad i \geq 0
$$

Os elementos pivô $v_{i}$ agem sobre as variáveis de Grassmann da seguinte maneira:

$$
v_{n}\left(x_{k}\right)= \begin{cases}0, & k<n \\ 1, & k=n ; \\ 0, & k=n+2 l-1, \quad l>0 \\ x_{n} x_{n+1} \cdots x_{n+2 l-2} x_{n+2 l-1}, & k=n+2 l, \quad l>0 .\end{cases}
$$

Definimos as aplicações shift $\tau: \Lambda \rightarrow \Lambda$ e $\tau: \mathbf{W}(\Lambda) \rightarrow \mathbf{W}(\Lambda)$ por

$$
\tau\left(x_{i}\right)=x_{i+1}, \quad \tau\left(\partial_{x_{i}}\right)=\partial_{x_{i+1}}, \quad i \geq 0 .
$$

A aplicação $\tau$ é claramente um endomorfismo de $\mathbf{W}(\Lambda)$. Obtemos no resultado a seguir algumas relações básicas entre elementos pivô.

Lema 4.1 São válidas as seguintes relações, para $i \geq 0$ :

(i) $v_{i}^{2}=x_{i+1} v_{i+2}$;

(ii) $\left[v_{i}, v_{i}\right]=2 x_{i+1} v_{i+2}$;

(iii) $\left[v_{i}, v_{i+1}\right]=-x_{i} v_{i+2}$;

(iv) $\left[v_{i}^{2}, v_{i+1}\right]=-v_{i+2}$;

(v) $\left[v_{i}, v_{i+2}\right]=2 x_{i} x_{i+1} x_{i+3} v_{i+4}$.

Demonstração: Para a prova do item (i), observe que

$$
v_{i}^{2}=\left(\partial_{x_{i}}+x_{i} x_{i+1} v_{i+2}\right)^{2}=\left(\partial_{x_{i}}\right)^{2}+\left(x_{i} x_{i+1} v_{i+2}\right)^{2}+\left[\partial_{x_{i}}, x_{i} x_{i+1} v_{i+2}\right]=x_{i+1} v_{i+2}
$$

O item (ii) segue do fato de que $\left[v_{i}, v_{i}\right]=2 v_{i}^{2}$. Observe também que

$$
\begin{aligned}
{\left[v_{i}, v_{i+1}\right] } & =\left[\partial_{x_{i}}+x_{i} x_{i+1} v_{i+2}, \partial_{x_{i+1}}+x_{i+1} x_{i+2} v_{i+3}\right] \\
& =\left[x_{i} x_{i+1} v_{i+2}, \partial_{x_{i+1}}\right]=-x_{i} v_{i+2}
\end{aligned}
$$


o que prova o item (iii). Além disso, temos

$$
\left[v_{i}^{2}, v_{i+1}\right]=\left[v_{i},\left[v_{i}, v_{i+1}\right]\right]=\left[v_{i},-x_{i} v_{i+2}\right]=-v_{i+2}
$$

e, portanto, vale o item (iv). Por fim, as igualdades

$$
\left[v_{i}, v_{i+2}\right]=\left[\partial_{x_{i}}+x_{i} x_{i+1} v_{i+2}, v_{i+2}\right]=x_{i} x_{i+1}\left[v_{i+2}, v_{i+2}\right]=2 x_{i} x_{i+1} x_{i+3} v_{i+4}
$$

tornam válido o item (v).

O lema a seguir mostra como calcular o produto supercomutador entre dois elementos pivô quaisquer, generalizando os itens (ii), (iii) e (v) do lema acima. Sua demonstração pode ser feita através de uma simples indução.

Lema 4.2 Para quaisquer inteiros $i, k \geq 0$, temos:

(i) se $k$ é par, então $\left[v_{i}, v_{i+k}\right]=2 x_{i} \cdots x_{i+k-1} x_{i+k+1} v_{i+k+2}$;

(ii) se $k$ é ímpar, então $\left[v_{i}, v_{i+k}\right]=-x_{i} \cdots x_{i+k-1} v_{i+k+1}$.

Demonstração: Se $k$ é par, obtemos através do item (ii) do Lema 4.1

$\left[v_{i}, v_{i+k}\right]=\left[\partial_{x_{i}}+x_{i} x_{i+1} \partial_{i+2}+\cdots+x_{i} \cdots x_{i+k-1} v_{i+k}, v_{i+k}\right]=2 x_{i} \cdots x_{i+k-1} x_{i+k+1} v_{i+k+2}$

provando a afirmação (i). Por outro lado, se $k$ é ímpar, pelo item (iii) do Lema 4.1 obtemos

$$
\left[v_{i}, v_{i+k}\right]=\left[\partial_{x_{i}}+x_{i} x_{i+1} \partial_{i+2}+\cdots+x_{i} \cdots x_{i+k-2} v_{i+k-1}, v_{i+k}\right]=-x_{i} \cdots x_{i+k-1} v_{i+k+1}
$$

o que prova a afirmação (ii).

A noção de autossimilaridade é de fundamental importância na teoria de grupos [18]. A álgebra de Lie de Fibonacci é autossimilar [36]. Uma definição geral foi dada por Bartholdi [6]. Uma álgebra de Lie $L$ é dita autossimilar se existe um homomorfismo

$$
\psi: L \rightarrow \operatorname{Der} R \curlywedge(R \otimes L)
$$

onde $R$ é uma álgebra comutativa e Der $R$ é a álgebra de Lie das derivações de $R$. Essa definição estende-se facilmente para superálgebras, e consideramos $R$ uma superálgebra associativa supercomutativa. 
Consideremos as seguintes superálgebras de Lie geradas por dois elementos: $L_{i}=$ $\operatorname{Lie}\left(v_{i}, v_{i+1}\right), i \geq 0$. Em particular, temos $L_{0}=\mathbf{R}$.

\section{Corolário 4.3 Temos}

(i) $v_{i} \in \mathbf{R}, i \geq 0$;

(ii) $\tau^{i}: \mathbf{R} \rightarrow L_{i}$ é um isomorfismo para todo $i \geq 0$;

(iii) $\mathbf{R}$ tem dimensão infinita;

(iv) existe uma imersão de autossimilaridade:

$$
\mathbf{R} \hookrightarrow\left\langle\partial_{x_{0}}\right\rangle_{K} \curlywedge\left(\Lambda\left[x_{0}\right] \otimes \tau(\mathbf{R})\right)
$$

\subsection{Base de $\mathrm{R}$}

Nosso objetivo agora é encontrar uma base para a superálgebra de Lie $\mathbf{R}=\operatorname{Lie}\left(v_{0}, v_{1}\right)$. Para simplificar a notação, denotaremos por $r_{n}$ um monômio cauda:

$$
r_{n}=x_{0}^{\xi_{0}} \cdots x_{n}^{\xi_{n}} \in \Lambda, \quad \xi_{i} \in\{0,1\} ; \quad n \geq 0 .
$$

Para $n<0$, vamos assumir que $r_{n}=1$. Se necessário, denotaremos outros elementos do tipo (4.1) por $r_{n}^{\prime}, r_{n}^{\prime \prime}$, etc.

Chamamos de monômio standard do primeiro tipo todo monômio da forma

$$
r_{n-2} v_{n}, \quad n \geq 0 .
$$

Chamamos de monômio standard do segundo tipo todo monômio da forma

$$
r_{n-3} x_{n-1} v_{n}, \quad n \geq 2,
$$

com exceção do monômio $x_{0} x_{2} v_{3}$, denominado monômio falso. Temos o seguinte resultado:

Lema 4.4 Seja char $K \neq 2$. Todos os seguintes monômios pertencem à superálgebra de Lie $\mathbf{R}=\operatorname{Lie}\left(v_{0}, v_{1}\right)$ : 
(i) monômios do primeiro tipo:

$$
\left\{r_{n-2} v_{n} \mid n \geq 0\right\}
$$

(ii) monômios do segundo tipo:

$$
\left\{r_{n-3} x_{n-1} v_{n} \mid n \geq 2\right\} \backslash\left\{x_{0} x_{2} v_{3}\right\}
$$

Demonstração: De agora em diante, o número $n$ será o comprimento, $v_{n}$ será a cabeça, $r_{n-2}$ a cauda, e os produtos remanescentes serão o pescoço do monômio referido.

Provaremos, por indução sobre o comprimento $n$, que todos os monômios do primeiro tipo pertencem a $\mathbf{R}$. Os monômios deste tipo com comprimento $n=0$ ou $n=1, v_{0} \mathrm{e}$ $v_{1}$, claramente pertencem a $\mathbf{R}=\operatorname{Lie}\left(v_{0}, v_{1}\right)$. Consideremos agora $n \geq 2$ e suponhamos que todo elemento da forma $r_{k-2} v_{k}$ com $0 \leq k \leq n-1$ pertence a $\mathbf{R}$. Observando os produtos

$$
\begin{gathered}
{\left[v_{n-2}^{2}, r_{n-3} v_{n-1}\right]=r_{n-3}\left[v_{n-2}^{2}, v_{n-1}\right]=-r_{n-3} v_{n},} \\
{\left[v_{n-2}, r_{n-3} v_{n-1}\right]= \pm r_{n-3}\left[v_{n-2}, v_{n-1}\right]= \pm r_{n-3} x_{n-2} v_{n}}
\end{gathered}
$$

e tendo em vista que $v_{n-2}, r_{n-3} v_{n-1} \in \mathbf{R}$, concluímos que todos os monômios do primeiro tipo de comprimento $n$ pertencem à superálgebra $\mathbf{R}$.

Em relação aos monômios do segundo tipo, é fácil ver que $x_{1} v_{2}$ e $x_{2} v_{3}$ pertencem a $\mathbf{R}$, uma vez que $\left[v_{0}, v_{0}\right]=2 x_{1} v_{2},\left[v_{1}, v_{1}\right]=2 x_{2} v_{3}$ e char $K \neq 2$. Consideremos agora monômios do segundo tipo de comprimento maior ou igual a 4, i.e., elementos da forma $r_{n-3} x_{n-1} v_{n}$ com $n \geq 4$, separando-os nos seguintes casos:

A) Se $n$ é par, o Lema 4.2 nos mostra que

$$
\left[v_{0}, v_{n-2}\right]=2 x_{0} x_{1} \cdots x_{n-3} x_{n-1} v_{n}
$$

Sendo $v_{0}$ e $v_{n-2}$ monômios do primeiro tipo, já sabemos que ambos pertencem a $\mathbf{R}$ e, como char $K \neq 2$, concluímos que

$$
x_{0} x_{1} \cdots x_{n-3} x_{n-1} v_{n} \in \mathbf{R} .
$$

Multiplicando esse elemento por $v_{i}(0 \leq i \leq n-3)$, podemos eliminar qualquer fator $x_{i}$. Logo, todo monômio do segundo tipo de comprimento par $n$ pertence a $\mathbf{R}$. 
B) Se $n$ é ímpar, o Lema 4.2 também nos dá que

$$
\left[v_{1}, x_{0} v_{n-2}\right]=-x_{0}\left[v_{1}, v_{n-2}\right]=-2 x_{0} x_{1} x_{2} \cdots x_{n-3} x_{n-1} v_{n}
$$

Uma vez que $v_{1}$ e $x_{0} v_{n-2}$ pertencem a $\mathbf{R}$ (são monômios do primeiro tipo) e char $K \neq 2$, também é verdade que

$$
x_{0} x_{1} x_{2} \cdots x_{n-3} x_{n-1} v_{n} \in \mathbf{R} .
$$

Multiplicando esse elemento por $v_{i}(0 \leq i \leq n-3)$, podemos eliminar qualquer fator $x_{i}$. Portanto, todo monômio do segundo tipo de comprimento ímpar $n$ pertence a $\mathbf{R}$.

É importante observar que a suposição de que char $K \neq 2$ foi necessária apenas para provar que os elementos do segundo tipo pertencem a $\mathbf{R}$. A afirmação sobre os monômios do primeiro tipo permanece válida quando char $K=2$.

Veremos no teorema abaixo que os monômios apresentados no Lema 4.4 constituem, na verdade, uma base para a superálgebra de Lie $\mathbf{R}=\operatorname{Lie}\left(v_{0}, v_{1}\right)$ quando char $K \neq 2$.

Teorema 4.5 Seja char $K \neq 2$. Uma base da superálgebra de Lie $\mathbf{R}=\operatorname{Lie}\left(v_{0}, v_{1}\right)$ é dada pelos monômios standard de primeiro e segundo tipos apresentados no Lema 4.4.

Demonstração: Pelo Lema 4.4, os monômios referidos pertencem a R. Para mostrar que eles geram $\mathbf{R}$ como espaço vetorial, é suficiente provar que qualquer produto supercomutador entre monômios standard é combinação linear de monômios standard. Alguns esforços abaixo são necessários para verificar que os monômios do segundo tipo não contêm o monômio falso $x_{0} x_{2} v_{3}$.

Nos produtos que consideraremos a seguir, é possível que os monômios que os compõem contenham um mesmo fator $x_{i}$, resultando em 0 , que é combinação linear de monômios de primeiro ou segundo tipo, como desejado. Para simplificar os cálculos, vamos supor que os fatores $x_{i}$ desses monômios sejam todos distintos. Em alguns momentos, escreveremos elementos de primeiro ou segundo tipo na forma mais geral $r_{n-1} v_{n}$.

1) Consideremos produtos de monômios standard de mesmo comprimento $n$. Observe que

$$
\left[r_{n-1} v_{n}, r_{n-1}^{\prime} v_{n}\right]= \pm 2 r_{n-1}^{\prime \prime} x_{n+1} v_{n+2}
$$


é múltiplo de um monômio do segundo tipo, exceto eventualmente para $n=1$, devido à possibilidade de obtermos um múltiplo do monômio falso. Mas podemos descartar essa possibilidade, uma vez que, para $n=1$, o único produto possível em (4.4) é $\left[v_{1}, v_{1}\right]=2 x_{2} v_{3}$.

2) Consideremos agora produtos de monômios standard de comprimentos distintos $n<m, \operatorname{com} n \equiv m(\bmod 2)$. Nesse caso, temos

$$
v_{n}=\partial_{x_{n}}+x_{n} x_{n+1}\left(\partial_{x_{n+2}}+\cdots+x_{m-4} x_{m-3}\left(\partial_{x_{m-2}}+x_{m-2} x_{m-1} v_{m}\right) \cdots\right) .
$$

2.a) Se o monômio da direita é do primeiro tipo, o produto

$$
\begin{aligned}
{\left[r_{n-1} v_{n}, r_{m-2}^{\prime} v_{m}\right]=} & r_{n-1}\left(\partial_{x_{n}}\left(r_{m-2}^{\prime}\right)+x_{n} x_{n+1} \partial_{x_{n+2}}\left(r_{m-2}^{\prime}\right)\right. \\
& \left.+\ldots+x_{n} \cdots x_{m-3} \partial_{x_{m-2}}\left(r_{m-2}^{\prime}\right)\right) v_{m} \\
& \pm r_{n-1} x_{n} x_{n+1} \cdots x_{m-2} x_{m-1} r_{m-2}^{\prime}\left[v_{m}, v_{m}\right] \\
= & \left(\sum_{j} \pm r_{m-2}^{(j)}\right) v_{m} \pm 2 r_{m-1}^{\prime \prime} x_{m+1} v_{m+2}
\end{aligned}
$$

resulta numa combinação linear de monômios de primeiro e segundo tipos (uma vez que $m \geq 2, r_{m-1}^{\prime \prime} x_{m+1} v_{m+2}$ não pode ser o monômio falso, pois tem comprimento $m+2 \geq 4$ );

2.b) Se o monômio da direita é do segundo tipo, todos os produtos possíveis (aqui usamos a mesma apresentação (4.5) de $v_{n}$ )

$$
\begin{aligned}
(m=2) & {\left[v_{0}, x_{1} v_{2}\right]=\left[\partial_{x_{0}}+x_{0} x_{1} v_{2}, x_{1} v_{2}\right]=0, } \\
{\left[v_{1}, x_{2} v_{3}\right] } & =\left[\partial_{x_{1}}+x_{1} x_{2} v_{3}, x_{2} v_{3}\right]=0, \\
& \\
{\left[r_{n-1} v_{n}, r_{m-3}^{\prime} x_{m-1} v_{m}\right]=} & r_{n-1}\left(\partial_{x_{n}}\left(r_{m-3}^{\prime} x_{m-1}\right)+x_{n} x_{n+1} \partial_{x_{n+2}}\left(r_{m-3}^{\prime} x_{m-1}\right)\right. \\
& \left.+\ldots+x_{n} \cdots x_{m-3} \partial_{x_{m-2}}\left(r_{m-3}^{\prime} x_{m-1}\right)\right) v_{m} \\
& \pm r_{n-1} x_{n} x_{n+1} \cdots x_{m-2} x_{m-1} r_{m-3}^{\prime} x_{m-1}\left[v_{m}, v_{m}\right] \\
= & \left(\sum_{j} \pm r_{m-3}^{(j)}\right) x_{m-1} v_{m}
\end{aligned}
$$

resultam numa combinação linear de monômios do segundo tipo.

3) Por fim, vamos considerar produtos de monômios de comprimentos distintos $n<m$, com $n \equiv m-1(\bmod 2)$. Como $n<m$, temos

$$
v_{n}=\partial_{x_{n}}+x_{n} x_{n+1}\left(\partial_{x_{n+2}}+\cdots+x_{m-5} x_{m-4}\left(\partial_{x_{m-3}}+x_{m-3} x_{m-2} v_{m-1}\right) \cdots\right) .
$$


3.a) Se o monômio da direita é do primeiro tipo, o produto

$$
\begin{aligned}
{\left[r_{n-1} v_{n}, r_{m-2}^{\prime} v_{m}\right]=} & r_{n-1}\left(\partial_{x_{n}}\left(r_{m-2}^{\prime}\right)+x_{n} x_{n+1} \partial_{x_{n+2}}\left(r_{m-2}^{\prime}\right)\right. \\
& \left.+\ldots+x_{n} \cdots x_{m-4} \partial_{x_{m-3}}\left(r_{m-2}^{\prime}\right)\right) v_{m} \\
& \pm r_{n-1} x_{n} x_{n+1} \cdots x_{m-3} x_{m-2} r_{m-2}^{\prime}\left[v_{m-1}, v_{m}\right] \\
= & \left(\sum_{j} \pm r_{m-2}^{(j)}\right) v_{m} \pm r_{m-2}^{\prime \prime} x_{m-1} v_{m+1}
\end{aligned}
$$

resulta numa combinação linear de monômios do primeiro tipo;

3.b) Se o monômio da direita é do segundo tipo, todos os casos possíveis (onde usamos a apresentação (4.6))

$$
\begin{aligned}
&(m=2) \quad\left[v_{1}, x_{1} v_{2}\right]=\left[\partial_{x_{1}}+x_{1} x_{2} v_{3}, x_{1} v_{2}\right]=v_{2} \\
&(m=3) \quad\left[v_{0}, x_{2} v_{3}\right]=\left[\partial_{x_{0}}+x_{0} x_{1} \partial_{x_{2}}+x_{0} x_{1} x_{2} x_{3} v_{4}, x_{2} v_{3}\right]=x_{0} x_{1} v_{3}, \\
& {\left[v_{2}, x_{2} v_{3}\right]=\left[\partial_{x_{2}}+x_{2} x_{3} v_{4}, x_{2} v_{3}\right]=v_{3} } \\
&\left.\quad r_{n-1} v_{n}, r_{m-3}^{\prime} x_{m-1} v_{m}\right]= r_{n-1}\left(\partial_{x_{n}}\left(r_{m-3}^{\prime} x_{m-1}\right)+x_{n} x_{n+1} \partial_{x_{n+2}}\left(r_{m-3}^{\prime} x_{m-1}\right)\right. \\
&\left.+\ldots+x_{n} \cdots x_{m-4} \partial_{x_{m-3}}\left(r_{m-3}^{\prime} x_{m-1}\right)\right) v_{m} \\
&(m \geq 4) \quad \pm r_{n-1} x_{n} \cdots x_{m-2} r_{m-3}^{\prime}\left[v_{m-1}, x_{m-1} v_{m}\right] \\
&=\left(\sum_{j} \pm r_{m-3}^{(j)}\right) x_{m-1} v_{m} \pm r_{m-2}^{\prime \prime} v_{m}
\end{aligned}
$$

também resultam em combinações lineares de monômios de primeiro ou segundo tipo.

Uma vez que os monômios dos dois tipos são linearmente independentes em Der $\Lambda$ (veja [36]), eles formam uma base para a superálgebra de $\operatorname{Lie} \mathbf{R}=\operatorname{Lie}\left(v_{0}, v_{1}\right)$.

Como consequência do teorema acima e da demonstração do Lema 4.4, podemos obter uma base para $\mathbf{R}$ no caso em que char $K=2$.

Corolário 4.6 Seja char $K=2$. Os monômios standard do primeiro tipo e os quadrados dos elementos pivô, $\left\{x_{n-1} v_{n} \mid n \geq 2\right\}$, formam uma base tanto para a superálgebra de Lie $\mathbf{R}=\operatorname{Lie}\left(v_{0}, v_{1}\right)$ como para a superálgebra de Lie restrita $\operatorname{Lie}_{p}\left(v_{0}, v_{1}\right)$.

Demonstração: Seja $L=L_{\overline{0}} \oplus L_{\overline{1}} \subset \mathbf{W}(\Lambda)$ a subálgebra gerada por $v_{0}, v_{1}$ considerando apenas o colchete supercomutador $[$,$] . Pelo teorema acima e pela demonstração do$ Lema 4.4, sua base $\left\{w_{j} \mid j \in J\right\}$ consiste de todos os monômios do primeiro tipo. Para obter a álgebra de Lie restrita $\operatorname{Lie}_{p}\left(v_{0}, v_{1}\right)=\operatorname{Lie}_{p}(L)$, é suficiente adicionar todas 
as potências $\left\{w_{j}^{\left[p^{n}\right]} \mid n \geq 1, j \in J\right\}$. Essas potências são triviais, a menos que sejam quadrados de elementos pivô.

Consideremos agora o caso da superálgebra de Lie $\mathbf{R}=\operatorname{Lie}\left(v_{0}, v_{1}\right)$. Neste caso, precisamos adicionar os quadrados de uma base da componente ímpar $L_{\overline{1}}$. Novamente, adicionamos os mesmos quadrados dos elementos pivô.

\subsection{Funções peso}

Suponhamos agora que as superderivações e as variáveis de Grassmann tenham peso wt como segue:

$$
\operatorname{wt}\left(\partial_{x_{i}}\right)=-\operatorname{wt}\left(x_{i}\right)=\alpha_{i} \in \mathbb{C}, \quad i \geq 0 .
$$

Queremos que todos os termos na relação recursiva

$$
v_{i}=\partial_{x_{i}}+x_{i} x_{i+1} v_{i+2}, \quad i \geq 0
$$

tenham o mesmo peso e que, além disso, a função peso seja aditiva em monômios nas letras $\left\{x_{i}, \partial_{x_{i}}, v_{i} \mid i \geq 0\right\}$. Então, para cada $i \geq 0$ temos

$$
\operatorname{wt}\left(v_{i}\right)=\operatorname{wt}\left(\partial_{x_{i}}\right)=\alpha_{i}, \quad \alpha_{i}=-\alpha_{i}-\alpha_{i+1}+\alpha_{i+2} .
$$

Obtemos a relação de recorrência

$$
\alpha_{i+2}=2 \alpha_{i}+\alpha_{i+1}, \quad i \geq 0
$$

$\mathrm{Na}$ forma matricial,

$$
\left(\begin{array}{c}
\alpha_{i+1} \\
\alpha_{i+2}
\end{array}\right)=A\left(\begin{array}{c}
\alpha_{i} \\
\alpha_{i+1}
\end{array}\right), \quad i \geq 0 ; \quad \text { onde } A=\left(\begin{array}{cc}
0 & 1 \\
2 & 1
\end{array}\right)
$$

Os autovalores da matriz $A$ (raízes do polinômio característico de (4.7)) são $\lambda_{1}=2 \mathrm{e}$ $\lambda_{2}=-1$. Então, as sequências $\alpha_{i}^{(1)}=2^{i}(i \geq 0)$ e $\alpha_{i}^{(2)}=(-1)^{i}(i \geq 0)$ formam uma base para o espaço de soluções da relação de recorrência (4.7). Dessa forma, podemos introduzir duas funções peso como segue.

Lema 4.7 Temos as seguintes funções peso: 
(i) $\operatorname{wt}\left(\partial_{x_{n}}\right)=\operatorname{wt}\left(v_{n}\right)=2^{n}, n \geq 0$ (a função peso);

(ii) $\operatorname{swt}\left(\partial_{x_{n}}\right)=\operatorname{swt}\left(v_{n}\right)=(-1)^{n}, n \geq 0$ (a função superpeso);

(iii) $\operatorname{Wt}\left(v_{n}\right)=\left(\operatorname{wt}\left(v_{n}\right), \operatorname{swt}\left(v_{n}\right)\right)=\left(2^{n},(-1)^{n}\right), n \geq 0$ (a função vetor peso).

Demonstração: Segue dos comentários feitos acima.

De agora em diante, chamaremos simplesmente de monômios quaisquer monômios nas letras $\left\{x_{i}, \partial_{x_{i}}, v_{i} \mid i \geq 0\right\}$, pertencentes à álgebra associativa End $\Lambda$.

Lema 4.8 As funções peso estão bem definidas em todos os monômios. Além disso, são aditivas em produtos de monômios, i.e., se a e b são dois monômios desse tipo, então

$$
\mathrm{Wt}(a \cdot b)=\mathrm{Wt}(a)+\mathrm{Wt}(b) .
$$

Demonstração: Segue da forma como construímos as funções peso.

\section{4 $\mathbb{Z}^{2}$-graduação de $R$; coordenadas peso e multi- grau}

Lema 4.9 As álgebras $\mathbf{R}=\operatorname{Lie}\left(v_{0}, v_{1}\right)$ e $\mathbf{A}=\operatorname{Alg}\left(v_{0}, v_{1}\right)$ são $\mathbb{Z}^{2}$-graduadas pelo multigrau em $\left\{v_{0}, v_{1}\right\}$ :

$$
\mathbf{R}=\underset{n_{1}, n_{2} \geq 0}{\oplus} \mathbf{R}_{n_{1} n_{2}}, \quad \mathbf{A}=\underset{n_{1}, n_{2} \geq 0}{\bigoplus} \mathbf{A}_{n_{1} n_{2}}
$$

Demonstração: Os vetores peso $\operatorname{Wt}\left(v_{0}\right)=(1,1)$ e $\operatorname{Wt}\left(v_{1}\right)=(2,-1)$ dados no Lema 4.7 são linearmente independentes. Obtemos, então, um reticulado peso:

$$
\Gamma=\mathbb{Z} \mathrm{Wt}\left(v_{0}\right) \oplus \mathbb{Z} \mathrm{Wt}\left(v_{1}\right) \subset \mathbb{R}^{2}
$$

Para $n_{1}, n_{2} \geq 0$, seja $\mathbf{A}_{n_{1} n_{2}} \subset \mathbf{A}$ o subespaço gerado por todos os monômios de graus $n_{1}$ e $n_{2}$ em relação aos geradores $v_{0}$ e $v_{1}$, respectivamente. Pelo Lema 4.8, para todo $v \in \mathbf{A}_{n_{1} n_{2}}$, temos

$$
\mathrm{Wt}(v)=n_{1} \mathrm{Wt}\left(v_{0}\right)+n_{2} \mathrm{Wt}\left(v_{1}\right)=\left(n_{1}+2 n_{2}, n_{1}-n_{2}\right) .
$$


Como os vetores $\mathrm{Wt}\left(v_{0}\right)$ e $\mathrm{Wt}\left(v_{1}\right)$ são linearmente independentes, concluímos que elementos pertencentes a componentes $\mathbf{A}_{n_{1} n_{2}}$ distintas são linearmente independentes.

A prova para a superálgebra de Lie $\mathbf{R}=\operatorname{Lie}\left(v_{0}, v_{1}\right)$ é análoga.

Observe que se $v \in \mathbf{A}_{n_{1} n_{2}}$, então $\operatorname{swt}(v)=n_{1} \operatorname{swt}\left(v_{0}\right)+n_{2} \operatorname{swt}\left(v_{1}\right)=n_{1}-n_{2}=0$ se, e somente se, $n_{1}=n_{2}$. A diagonal da $\mathbb{Z}^{2}$-graduação de $\mathbf{A}$ é definida como sendo a soma direta das componentes homogêneas cujos elementos têm superpeso nulo, ou seja, a subálgebra $\underset{n \geq 0}{\oplus} \mathbf{A}_{n n}$. Analogamente, a diagonal da $\mathbb{Z}^{2}$-graduação de $\mathbf{R}$ é dada pela soma direta $\underset{n \geq 0}{\bigoplus} \mathbf{R}_{n n}$.

Corolário 4.10 Valem as seguintes afirmações:

(i) As álgebras $\mathbf{R}$ e $\mathbf{A}$ possuem decomposição triangular em somas diretas de três subálgebras:

$$
\mathbf{R}=\mathbf{R}_{+} \oplus \mathbf{R}_{0} \oplus \mathbf{R}_{-}, \quad \mathbf{A}=\mathbf{A}_{+} \oplus \mathbf{A}_{0} \oplus \mathbf{A}_{-},
$$

onde as componentes são geradas por monômios de superpeso positivo, zero e negativo, respectivamente;

(ii) As componentes nulas $\mathbf{R}_{0}$ e $\mathbf{A}_{0}$ coincidem com as diagonais correspondentes $\mathbf{R}_{0}=$ $\bigoplus_{n \geq 0} \mathbf{R}_{n n}$ e $\mathbf{A}_{0}=\underset{n \geq 0}{\bigoplus} \mathbf{A}_{n n}$.

Demonstração: Segue da aditividade da função superpeso.

Dado um monômio não nulo $v \in \mathbf{A}_{n_{1} n_{2}}, n_{1}, n_{2} \geq 0$, definimos o vetor multigrau

$$
\operatorname{Gr}(v)=\left(n_{1}, n_{2}\right) \in \mathbb{Z}^{2} \subset \mathbb{R}^{2}
$$

Podemos identificá-lo no plano $\mathbb{R}^{2}$ com coordenadas standard $\left(X_{1}, X_{2}\right)$, as quais chamamos de coordenadas multigrau, fazendo $\operatorname{Gr}(v)=\left(n_{1}, n_{2}\right)=\left(X_{1}, X_{2}\right)$.

Seja $\left(X_{1}, X_{2}\right) \in \mathbb{R}^{2}$ um ponto qualquer do plano. Introduzimos também as coordenadas peso $\left(Z_{1}, Z_{2}\right)$ no plano $\mathbb{R}^{2}$ da seguinte maneira:

$$
\left\{\begin{array} { l } 
{ Z _ { 1 } = X _ { 1 } + 2 X _ { 2 } ; } \\
{ Z _ { 2 } = X _ { 1 } - X _ { 2 } ; }
\end{array} \quad \left\{\begin{array}{l}
X_{1}=\left(Z_{1}+2 Z_{2}\right) / 3 \\
X_{2}=\left(Z_{1}-Z_{2}\right) / 3
\end{array}\right.\right.
$$


Lema 4.11 Seja $v \in \mathbf{A}$ um monômio, $\operatorname{Gr}(v)=\left(X_{1}, X_{2}\right)$ o seu multigrau, e $\left(Z_{1}, Z_{2}\right)$ as suas coordenadas peso. Então, $\left(Z_{1}, Z_{2}\right)=\mathrm{Wt}(v)=(\operatorname{wt}(v), \operatorname{swt}(v))$.

Demonstração: Através de (4.9), colocamos o peso e o superpeso em função do multigrau, o que nos dá

$$
\operatorname{wt}(v)=n_{1}+2 n_{2}=X_{1}+2 X_{2}=Z_{1}, \quad \operatorname{swt}(v)=n_{1}-n_{2}=X_{1}-X_{2}=Z_{2} .
$$

Lema 4.12 São válidas as seguintes afirmações:

(i) O multigrau de um elemento pivô $v_{n}$ é dado por

$$
\operatorname{Gr}\left(v_{n}\right)=\frac{1}{3}\left(2^{n}+2(-1)^{n}, 2^{n}-(-1)^{n}\right), \quad n \geq 0
$$

(ii) Os elementos $v_{n}$ pertencem à união de duas retas no plano: $X_{1}-X_{2}=1 e$ $X_{1}-X_{2}=-1$.

Demonstração: O item (i) segue do fato de que $\mathrm{Wt}\left(v_{n}\right)=\left(2^{n},(-1)^{n}\right)$ e das relações entre as coordenadas (4.10). Para a verificação do item (ii), observemos que para um elemento pivô $v_{n}$ temos $X_{1}-X_{2}=Z_{2}=\operatorname{swt}\left(v_{n}\right)=(-1)^{n}$. Assim, se $n$ é par, $v_{n}$ pertence à reta $X_{1}-X_{2}=1$. Por outro lado, se $n$ é ímpar, $v_{n}$ está localizado na reta $X_{1}-X_{2}=-1$.

Para exemplificar, listamos os multigraus e os vetores peso de todos os monômios standard de comprimento menor ou igual a 5: 
Monômio Vetor peso Multigrau

$\begin{array}{ccc}v_{0} & (1,1) & (1,0) \\ v_{1} & (2,-1) & (0,1) \\ v_{2} & (4,1) & (2,1) \\ x_{0} v_{2} & (3,0) & (1,1) \\ x_{1} v_{2} & (2,2) & (2,0) \\ v_{3} & (8,-1) & (2,3) \\ x_{0} v_{3} & (7,-2) & (1,3) \\ x_{1} v_{3} & (6,0) & (2,2) \\ x_{0} x_{1} v_{3} & (5,-1) & (1,2) \\ x_{2} v_{3} & (4,-2) & (0,2) \\ v_{4} & (16,1) & (6,5) \\ x_{0} v_{4} & (15,0) & (5,5) \\ x_{1} v_{4} & (14,2) & (6,4) \\ x_{2} v_{4} & (12,0) & (4,4) \\ x_{0} x_{1} v_{4} & (13,1) & (5,4) \\ x_{0} x_{2} v_{4} & (11,-1) & (3,4) \\ x_{1} x_{2} v_{4} & (10,1) & (4,3) \\ x_{0} x_{1} x_{2} v_{4} & (9,0) & (3,3) \\ x_{3} v_{4} & (8,2) & (4,2) \\ x_{0} x_{3} v_{4} & (7,1) & (3,2) \\ x_{1} x_{3} v_{4} & (6,3) & (4,1) \\ x_{0} x_{1} x_{3} v_{4} & (5,2) & (3,1) \\ v_{5} & (32,-1) & (10,11) \\ & & \end{array}$

Monômio Vetor peso Multigrau

$\begin{array}{ccc}x_{0} v_{5} & (31,-2) & (9,11) \\ x_{1} v_{5} & (30,0) & (10,10) \\ x_{2} v_{5} & (28,-2) & (8,10) \\ x_{3} v_{5} & (24,0) & (8,8) \\ x_{0} x_{1} v_{5} & (29,-1) & (9,10) \\ x_{0} x_{2} v_{5} & (27,-3) & (7,10) \\ x_{0} x_{3} v_{5} & (23,-1) & (7,8) \\ x_{1} x_{2} v_{5} & (26,-1) & (8,9) \\ x_{1} x_{3} v_{5} & (22,1) & (8,7) \\ x_{2} x_{3} v_{5} & (20,-1) & (6,7) \\ x_{0} x_{1} x_{2} v_{5} & (25,-2) & (7,9) \\ x_{0} x_{1} x_{3} v_{5} & (21,0) & (7,7) \\ x_{0} x_{2} x_{3} v_{5} & (19,-2) & (5,7) \\ x_{1} x_{2} x_{3} v_{5} & (18,0) & (6,6) \\ x_{0} x_{1} x_{2} x_{3} v_{5} & (17,-1) & (5,6) \\ x_{4} v_{5} & (16,-2) & (4,6) \\ x_{0} x_{4} v_{5} & (15,-3) & (3,6) \\ x_{1} x_{4} v_{5} & (14,-1) & (4,5) \\ x_{2} x_{4} v_{5} & (12,-3) & (2,5) \\ x_{0} x_{1} x_{4} v_{5} & (13,-2) & (3,5) \\ x_{0} x_{2} x_{4} v_{5} & (11,-4) & (1,5) \\ x_{1} x_{2} x_{4} v_{5} & (10,-2) & (2,4) \\ x_{0} x_{1} x_{2} x_{4} v_{5} & (9,-3) & (1,4) \\ & & \end{array}$

No próximo resultado, provaremos que o monômio falso $x_{0} x_{2} v_{3}$ não pertence à superálgebra de Lie $\mathbf{R}=\operatorname{Lie}\left(v_{0}, v_{1}\right)$ e, portanto, não pode ser listado entre os monômios standard.

Lema 4.13 Valem as seguintes afirmações:

(i) $O$ monômio falso tem multigrau $\operatorname{Gr}\left(x_{0} x_{2} v_{3}\right)=(-1,2)$;

(ii) O monômio falso não figura na lista dos monômios standard. 
Demonstração: Para a prova da afirmação (i), consideremos o elemento $v=x_{2} v_{3} \in \mathbf{R}$. Como vemos na tabela 4.11 , temos $\operatorname{Gr}(v)=(0,2)$. Uma vez que $\left[v_{0}, x_{0} x_{2} v_{3}\right]=v$ e a função multigrau é aditiva em produtos de monômios (Lema 4.8), temos $\operatorname{Gr}\left(x_{0} x_{2} v_{3}\right)=\operatorname{Gr}(v)-\operatorname{Gr}\left(v_{0}\right)=(0,2)-(1,0)=(-1,2)$. Então o monômio falso não pode ser obtido através de produtos sucessivos de $v_{0}$ e $v_{1}$, pois, caso contrário, suas coordenadas multigrau seriam ambas não negativas. Portanto, $x_{0} x_{2} v_{3}$ não pertence a R.

Pela expressão obtida no Lema 4.12 para o multigrau de um elemento pivô, podemos estabelecer uma relação entre os multigraus de $v_{n}$ e $\tau\left(v_{n}\right)=v_{n+1} . \operatorname{Se} \operatorname{Gr}\left(v_{n}\right)=\left(n_{1}, n_{2}\right)$, temos

$$
\begin{aligned}
\operatorname{Gr}\left(v_{n+1}\right) & =\frac{1}{3}\left(2^{n+1}+2(-1)^{n+1}, 2^{n+1}-(-1)^{n+1}\right) \\
& =\frac{1}{3}\left(2\left(2^{n}-(-1)^{n}\right), 2^{n}+2(-1)^{n}+2^{n}-(-1)^{n}\right) \\
& =\left(2 n_{2}, n_{1}+n_{2}\right) .
\end{aligned}
$$

Podemos generalizar essa relação para um elemento $v \in \mathbf{A}_{n_{1} n_{2}}$ e sua imagem pelo endomorfismo $\tau$ :

Lema 4.14 Seja $0 \neq v \in \mathbf{A}_{n_{1} n_{2}}, n_{1}, n_{2} \geq 0$, e seja $\tau(v)$ sua imagem pelo endomorfismo

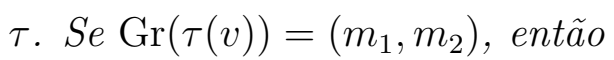

$$
\left(\begin{array}{c}
m_{1} \\
m_{2}
\end{array}\right)=\left(\begin{array}{c}
2 n_{2} \\
n_{1}+n_{2}
\end{array}\right)=A^{T} \cdot\left(\begin{array}{c}
n_{1} \\
n_{2}
\end{array}\right)
$$

onde $A=\left(\begin{array}{ll}0 & 1 \\ 2 & 1\end{array}\right)$ é a matriz definida na relação de recorrência (4.8).

Demonstração: Uma vez que $v \in \mathbf{A}_{n_{1} n_{2}}$, o elemento $v$ é uma combinação linear de produtos de $n_{1}$ fatores de $v_{0}$ e $n_{2}$ fatores de $v_{1}$. Então vale a igualdade

$$
\operatorname{Gr}(v)=n_{1} \operatorname{Gr}\left(v_{0}\right)+n_{2} \operatorname{Gr}\left(v_{1}\right) .
$$

Como $\tau$ é um endomorfismo, $\tau(v)$ é uma combinação linear de produtos de $n_{1}$ fatores de $\tau\left(v_{0}\right)$ e $n_{2}$ fatores de $\tau\left(v_{1}\right)$. Sabendo que $\tau\left(v_{0}\right)=v_{1}$ e $\tau\left(v_{1}\right)=v_{2}$, concluímos pela aditividade da função multigrau que

$$
\operatorname{Gr}(\tau(v))=n_{1} \operatorname{Gr}\left(v_{1}\right)+n_{2} \operatorname{Gr}\left(v_{2}\right)=n_{1}(0,1)+n_{2}(2,1)=\left(2 n_{2}, n_{1}+n_{2}\right) .
$$




\subsection{Limitações para os pesos}

O lema a seguir nos dá estimativas para os pesos dos monômios standard de $\mathbf{R}$.

Lema 4.15 Os pesos dos monômios standard de primeiro e segundo tipos satisfazem as seguintes desigualdades:

$$
\begin{gathered}
2^{n-1}<\operatorname{wt}\left(r_{n-2} v_{n}\right) \leq 2^{n}, \quad n \geq 0 ; \\
2^{n-2}<\operatorname{wt}\left(r_{n-3} x_{n-1} v_{n}\right) \leq 2^{n-1}, \quad n \geq 2 .
\end{gathered}
$$

Demonstração: Observe primeiramente que o peso de uma cauda $r_{m}=$ $x_{0}^{\xi_{0}} \cdots x_{m}^{\xi_{m}}, \quad \xi_{i} \in\{0,1\}$, tem as seguintes limitações:

$$
0 \geq \operatorname{wt}\left(r_{m}\right) \geq-\left(2^{0}+2^{1}+\cdots+2^{m}\right)=-\frac{2^{m+1}-1}{2-1}=-2^{m+1}+1, \quad m \geq 0 .
$$

Veja que, formalmente, essas desigualdades permanecem válidas para $m=-1$. A partir de (4.12), podemos obter estimativas para os pesos dos monômios standard dos dois tipos. Temos, então,

$$
2^{n} \geq \operatorname{wt}\left(r_{n-2} v_{n}\right) \geq-2^{n-1}+1+2^{n}=2^{n-1}+1>2^{n-1}, \quad n \geq 1 .
$$

Como $\operatorname{wt}\left(v_{0}\right)=1=2^{0}$, temos

$$
2^{n-1}<\operatorname{wt}\left(r_{n-2} v_{n}\right) \leq 2^{n}, \quad n \geq 0
$$

e está provada a primeira parte do lema. Para a prova da segunda parte, observe que são válidas as desigualdades

$$
2^{n-1}=2^{n}-2^{n-1} \geq \operatorname{wt}\left(r_{n-3} x_{n-1} v_{n}\right) \geq-2^{n-2}+1-2^{n-1}+2^{n}=2^{n-2}+1>2^{n-2},
$$

as quais nos dão

$$
2^{n-2}<\operatorname{wt}\left(r_{n-3} x_{n-1} v_{n}\right) \leq 2^{n-1}, \quad n \geq 2
$$


Lema 4.16 Os superpesos dos monômios standard de $\mathbf{R}$ têm as seguintes estimativas:

$$
\begin{gathered}
-\frac{n}{2}-\frac{1}{2} \leq \operatorname{swt}\left(r_{n-2} v_{n}\right) \leq \frac{n}{2}, \quad n \geq 1 ; \\
-\frac{n}{2}-\frac{3}{2} \leq \operatorname{swt}\left(r_{n-3} x_{n-1} v_{n}\right) \leq \frac{n}{2}+1, \quad n \geq 2 .
\end{gathered}
$$

Demonstração: Seja $w=r_{n-2} v_{n}$ um monômio standard do primeiro tipo, $\operatorname{com} n \geq 1$. Suponhamos que o comprimento $n$ de $w$ seja par e façamos $n=2 k$. Tomando $r_{n-2}=$ $x_{0}^{\xi_{0}} x_{1}^{\xi_{1}} \cdots x_{2 k-3}^{\xi_{2 k-3}} x_{2 k-2}^{\xi_{2 k-2}}, 0 \leq \xi_{i} \leq 1$, e considerando os superpesos

$$
\begin{gathered}
\operatorname{swt}\left(x_{0}\right)=\operatorname{swt}\left(x_{2}\right)=\cdots=\operatorname{swt}\left(x_{2 k-2}\right)=-1, \\
\operatorname{swt}\left(x_{1}\right)=\operatorname{swt}\left(x_{3}\right)=\cdots=\operatorname{swt}\left(x_{2 k-3}\right)=1, \\
\operatorname{swt}\left(v_{n}\right)=1,
\end{gathered}
$$

obtemos as seguintes desigualdades:

$$
\begin{gathered}
-k \leq \operatorname{swt}\left(r_{n-2}\right) \leq k-1 ; \\
-\frac{n}{2}+1=-k+1 \leq \operatorname{swt}\left(r_{n-2} v_{n}\right) \leq k=\frac{n}{2} .
\end{gathered}
$$

Vejamos agora o caso em que o comprimento de $w$ é ímpar, com $n=2 k+1$. Fazendo $r_{n-2}=x_{0}^{\xi_{0}} x_{1}^{\xi_{1}} \cdots x_{2 k-2}^{\xi_{2 k-2}} x_{2 k-1}^{\xi_{2 k-1}}, 0 \leq \xi_{i} \leq 1$, e considerando o $\operatorname{superpeso~} \operatorname{swt}\left(v_{2 k+1}\right)=-1$, temos

$$
\begin{aligned}
-k & \leq \operatorname{swt}\left(r_{n-2}\right) \leq k ; \\
-\frac{n}{2}-\frac{1}{2}=-k-1 & \leq \operatorname{swt}\left(r_{n-2} v_{n}\right) \leq k-1=\frac{n}{2}-\frac{3}{2} .
\end{aligned}
$$

Em ambos os casos, valem as desigualdades

$$
-\frac{n}{2}-\frac{1}{2} \leq \operatorname{swt}\left(r_{n-2} v_{n}\right) \leq \frac{n}{2}
$$

Consideremos agora um monômio standard do segundo tipo $w=r_{n-3} x_{n-1} v_{n}$, com $n \geq 2$. No caso em que $n=2 k$, tomando $r_{n-3}=x_{0}^{\xi_{0}} x_{1}^{\xi_{1}} \cdots x_{2 k-4}^{\xi_{2 k-4}} x_{2 k-3}^{\xi_{2 k-3}}, 0 \leq \xi_{i} \leq 1 \mathrm{e}$ usando $\operatorname{swt}\left(x_{2 k-1} v_{2 k}\right)=2$, temos

$$
\begin{gathered}
-k+1 \leq \operatorname{swt}\left(r_{n-3}\right) \leq k-1 \\
-\frac{n}{2}+3=-k+3 \leq \operatorname{swt}\left(r_{n-3} x_{n-1} v_{n}\right) \leq k+1=\frac{n}{2}+1 .
\end{gathered}
$$


Já no caso em que $n=2 k+1$, fazendo $r_{n-3}=x_{0}^{\xi_{0}} x_{1}^{\xi_{1}} \cdots x_{2 k-3}^{\xi_{2 k-3}} x_{2 k-2}^{\xi_{2 k-2}}$ e usando $\operatorname{swt}\left(x_{2 k} v_{2 k+1}\right)=-2$, ocorre

$$
\begin{aligned}
-k & \leq \operatorname{swt}\left(r_{n-3}\right) \leq k-1 \\
-\frac{n}{2}-\frac{3}{2}=-k-2 & \leq \operatorname{swt}\left(r_{n-3} x_{n-1} v_{n}\right) \leq k-3=\frac{n}{2}-\frac{7}{2} .
\end{aligned}
$$

Em ambos os casos, valem as desigualdades

$$
-\frac{n}{2}-\frac{3}{2} \leq \operatorname{swt}\left(r_{n-3} x_{n-1} v_{n}\right) \leq \frac{n}{2}+1
$$

Agora conseguimos obter estimativas mais gerais.

Lema 4.17 Seja w um monômio standard de comprimento $n \geq 0$. Então

$$
2^{n-2}<\operatorname{wt}(w) \leq 2^{n}, \quad-\frac{n}{2}-\frac{3}{2} \leq \operatorname{swt}(w) \leq \frac{n}{2}+1 .
$$

Demonstração: Segue diretamente do Lema 4.15 e do Lema 4.16.

As estimativas encontradas no Lema 4.17 nos dão informações referentes à representação geométrica dos monômios standard no plano $\mathbb{R}^{2}$ em termos das coordenadas peso $\left(Z_{1}, Z_{2}\right)$, como mostra o teorema a seguir.

Teorema 4.18 O conjunto dos pontos do plano associados aos monômios standard de $\mathbf{R}=\operatorname{Lie}\left(v_{0}, v_{1}\right)$ é limitado por duas curvas logarítmicas em termos das coordenadas peso $\operatorname{Wt}(w)=\left(Z_{1}, Z_{2}\right)$ :

$$
-\frac{1}{2} \log _{2} Z_{1}-\frac{5}{2}<Z_{2}<\frac{1}{2} \log _{2} Z_{1}+2
$$

Demonstração: Seja $w$ um monômio standard de comprimento $n \geq 0$. Pelo Lema 4.17 , vale a desigualdade $2^{n-2}<Z_{1}$, de onde obtemos $n<\log _{2} Z_{1}+2$. Pelo mesmo lema, conseguimos cotas para $Z_{2}=\operatorname{swt}(w)$ :

$$
-\frac{1}{2} \log _{2} Z_{1}-\frac{5}{2}<-\frac{n}{2}-\frac{3}{2} \leq Z_{2} \leq \frac{n}{2}+1<\frac{1}{2} \log _{2} Z_{1}+2 .
$$




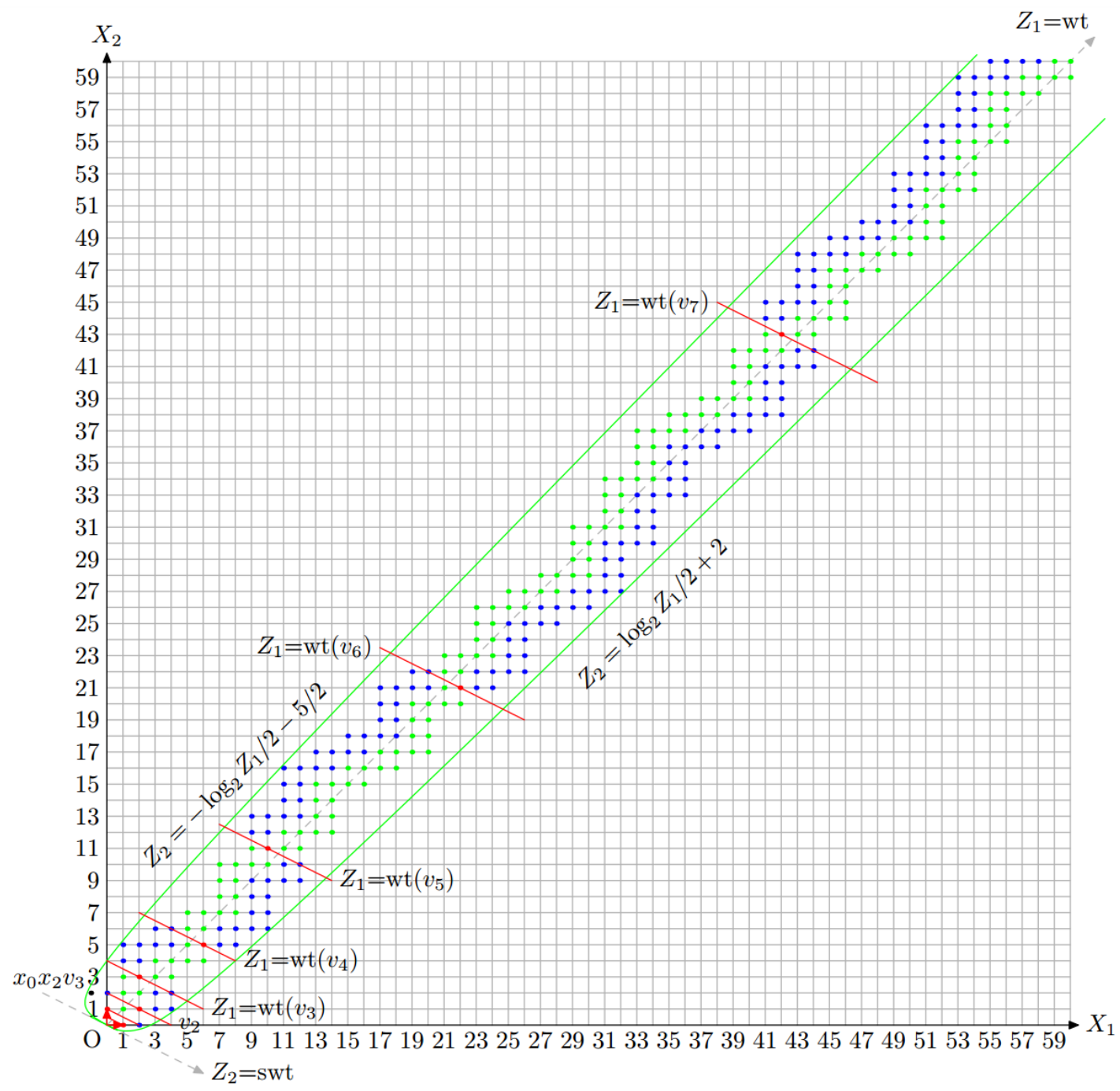

Figura 4.1: Representação dos monômios standard no plano. Os pontos verdes são monômios do primeiro tipo; os azuis, do segundo tipo. Os elementos pivô estão na cor vermelha, e o ponto de cor preta representa o monômio falso. As duas curvas logarítmicas que limitam os monômios standard estão na cor verde, enquanto as linhas vermelhas mostram pontos com pesos iguais aos de elementos pivô. O primeiro ponto vazio da diagonal é $(57,57)$. 


\subsection{Crescimento de $\mathrm{R}$ e $\mathrm{A}$}

O lema a seguir mostra que, para $m \geq 4$, existem exatamente dois monômios standard com peso igual a $m$.

Lema 4.19 Suponha char $K \neq 2$ e seja $m \geq 1$ um inteiro. Valem as seguintes afirmações:

(i) Existe um único monômio standard do primeiro tipo $w_{1}$ satisfazendo $\mathrm{wt}\left(w_{1}\right)=m$ (vale também para char $K=2$ );

(ii) Se $m \notin\{1,3\}$, existe um único monômio standard do segundo tipo $w_{2}$ satisfazendo $\operatorname{wt}\left(w_{2}\right)=m$;

(iii) Se $m \notin\{1,3\}$, existem exatamente dois monômios standard com peso igual a $m$; se $m=1$ ou $m=3$, existe apenas um monômio standard de peso $m$.

(iv) Se $m \notin\{1,3\}$ e $w_{1}=r_{n-2} v_{n}$, então $w_{2}=r_{n-2} x_{n} v_{n+1}$ (a cauda é a mesma);

(v) Se $m \notin\{1,3\}$, então $\operatorname{swt}\left(w_{1}\right)-\operatorname{swt}\left(w_{2}\right)=3 \cdot(-1)^{n}$;

(vi) Se $m \notin\{1,3\}$, então $\operatorname{Gr}\left(w_{1}\right)-\operatorname{Gr}\left(w_{2}\right)=(-1)^{n}(2,-1)$.

Demonstração: Primeiramente, vamos provar a afirmação (i) para $m=1$. Observe que se um monômio standard do primeiro tipo tem peso 1 , seu comprimento deve ser igual a 0 . De fato, se $r_{n-2} v_{n}$ é um monômio standard do primeiro tipo com comprimento $n \geq 1$, seu peso satisfaz $\operatorname{wt}\left(r_{n-2} v_{n}\right)>1$, em virtude do Lema 4.15. Assim, uma vez que $\operatorname{wt}\left(v_{0}\right)=1$, o único monômio standard do primeiro tipo com peso igual a 1 é $w_{1}=v_{0}$.

Agora provaremos a afirmação (i) para valores de $m$ maiores que 1 . Fixemos $n \geq 1$. Se $r_{n-2} v_{n}$ é um monômio standard do primeiro tipo de comprimento $n$, o Lema 4.15 nos mostra que $\operatorname{wt}\left(r_{n-2} v_{n}\right) \in\left\{2^{n-1}+1,2^{n-1}+2, \ldots, 2^{n}\right\}$ (o peso de $r_{n-2} v_{n}$ é claramente um número inteiro). Reciprocamente, para cada $m \in\left\{2^{n-1}+1,2^{n-1}+2, \ldots, 2^{n}\right\}$ existe um único monômio standard do primeiro tipo $w_{1}=r_{n-2} v_{n}$ tal que $\operatorname{wt}\left(w_{1}\right)=m$. Com efeito, uma vez que todo inteiro não negativo pode ser escrito unicamente como soma de potências de base 2 , para $c=2^{n}-m \in\left\{0, \ldots, 2^{n-1}-1\right\}$ existe uma única cauda $r_{n-2}=x_{0}^{\xi_{0}} \cdots x_{n-2}^{\xi_{n-2}}$ tal que $\operatorname{wt}\left(r_{n}\right)=-c$. Logo, existe um único $w_{1}=r_{n-2} v_{n}$ tal que $\operatorname{wt}\left(w_{1}\right)=2^{n}-c=m$. Assim, para todo $m \geq 2$ existe um único monômio standard do primeiro tipo $w_{1}$ satisfazendo $\operatorname{wt}\left(w_{1}\right)=m$. 
Para a prova da afirmação (ii), suponhamos inicialmente $m=2$. Com base no Lema 4.15, observamos que não existem monômios do segundo tipo de comprimento maior ou igual a 3 com peso $m=2$. Como $\operatorname{wt}\left(x_{1} v_{2}\right)=2, w_{1}=x_{1} v_{2}$ é o único monômio do segundo tipo cujo peso é igual a 2. Suponhamos agora $m=4$. O Lema 4.15 nos garante que só é possível haver monômios do segundo tipo de peso 4 se o comprimento for $n=3$, $\operatorname{logo} w_{1}=x_{2} v_{3}$ é o único monômio que satisfaz as condições desejadas.

Agora provaremos (ii) para valores de $m$ maiores que 4 . Fixemos $n \geq 4$. Se $r_{n-3} x_{n-1} v_{n}$ é um monômio standard do segundo tipo de comprimento $n$, do Lema 4.15 inferimos que wt $\left(r_{n-3} x_{n-1} v_{n}\right) \in\left\{2^{n-2}+1,2^{n-2}+2, \ldots, 2^{n-1}\right\}$. Com o mesmo argumento anterior concluímos também que, reciprocamente, para cada $m \in\left\{2^{n-2}+1,2^{n-2}+2, \ldots, 2^{n-1}\right\}$ existe um único monômio standard do segundo tipo $w_{2}=r_{n-3} x_{n-1} v_{n}$ para o qual $\operatorname{wt}\left(w_{2}\right)=m$. Então, para cada $m \geq 5$ existe um único monômio standard do segundo tipo $w_{2} \operatorname{com} \operatorname{wt}\left(w_{2}\right)=m$.

De (i) e (iii), concluímos que para $m \notin\{1,3\}$ existem exatamente dois monômios standard de peso $m$. Vejamos o que acontece nos casos $m=1$ e $m=3$. Pelo Lema 4.15, monômios do segundo tipo têm peso maior ou igual a 2. Então existe apenas um monômio standard com peso $m=1$, e este é do primeiro tipo: $w_{1}=v_{0}$. Por fim, uma procura por elementos da forma $r_{n-3} x_{n-1} v_{n}$ com peso $m=3$ apontaria apenas para o monômio falso $x_{0} x_{2} v_{3}$, que não é um monômio standard (Lema 4.13). Portanto, o único monômio standard com peso $m=3$ é $w_{1}=x_{0} v_{2}$ e provamos a afirmação (iii).

Para a prova de (iv), tomemos um inteiro positivo $m \notin\{1,3\}$ e seja $w_{1}=r_{n-2} v_{n}$ o monômio do primeiro tipo que satisfaz $\operatorname{wt}\left(w_{1}\right)=m$. Então $w_{2}=r_{n-2} x_{n} v_{n+1}$ é o monômio do segundo tipo que tem o mesmo peso, pois

$$
\begin{aligned}
\operatorname{wt}\left(r_{n-2} x_{n} v_{n+1}\right) & =\operatorname{wt}\left(r_{n-2}\right)+\operatorname{wt}\left(x_{n}\right)+\operatorname{wt}\left(v_{n+1}\right) \\
& =\operatorname{wt}\left(r_{n-2}\right)-2^{n}+2^{n+1} \\
& =\operatorname{wt}\left(r_{n-2}\right)+2^{n} \\
& =\operatorname{wt}\left(r_{n-2} v_{n}\right),
\end{aligned}
$$

onde usamos a aditividade da função peso (Lema 4.8). Isso implica que

$$
\begin{aligned}
\operatorname{swt}\left(w_{1}\right)-\operatorname{swt}\left(w_{2}\right) & =\operatorname{swt}\left(r_{n-2} v_{n}\right)-\operatorname{swt}\left(r_{n-2} x_{n} v_{n+1}\right) \\
& =\operatorname{swt}\left(r_{n-2}\right)+\operatorname{swt}\left(v_{n}\right)-\operatorname{swt}\left(r_{n-2}\right)-\operatorname{swt}\left(x_{n}\right)-\operatorname{swt}\left(v_{n+1}\right) \\
& =(-1)^{n}+(-1)^{n}-(-1)^{n+1} \\
& =3 \cdot(-1)^{n},
\end{aligned}
$$


o que prova o item (v). Por fim, pelo Lema 4.12, temos também

$$
\begin{aligned}
\operatorname{Gr}\left(w_{1}\right)-\operatorname{Gr}\left(w_{2}\right)= & \operatorname{Gr}\left(r_{n-2} v_{n}\right)-\operatorname{Gr}\left(r_{n-2} x_{n} v_{n+1}\right) \\
= & 2 \operatorname{Gr}\left(v_{n}\right)-\operatorname{Gr}\left(v_{n+1}\right) \\
= & \frac{2}{3}\left(2^{n}+2(-1)^{n}, 2^{n}-(-1)^{n}\right) \\
& -\frac{1}{3}\left(2^{n+1}+2(-1)^{n+1}, 2^{n+1}-(-1)^{n+1}\right) \\
= & (-1)^{n}(2,-1),
\end{aligned}
$$

provando assim a afirmação (vi).

No resultado abaixo, computamos a função de crescimento ponderada $\tilde{\gamma}_{\mathbf{R}}(m)$, tornando possível calcular a dimensão de Gelfand-Kirillov de $\mathbf{R}=\operatorname{Lie}\left(v_{0}, v_{1}\right)$. Obtemos também uma aproximação para a função de crescimento regular $\gamma_{\mathbf{R}}(m)$.

Teorema 4.20 Valem as seguintes afirmações:

(i) Se char $K \neq 2$, então $\mathbf{R}$ tem crescimento linear:

$$
\tilde{\gamma}_{\mathbf{R}}(m)=2 m-2, \quad m \geq 3 ;
$$

(ii) Se char $K=2$, então

$$
\tilde{\gamma}_{\mathbf{R}}(m)=m+\left[\log _{2} m\right], \quad m \geq 1
$$

(iii) $\operatorname{GKdim} \mathbf{R}=\underline{\operatorname{GKdim}} \mathbf{R}=1$;

(iv) $S e$ char $K \neq 2$, então

$$
\lim _{m \rightarrow \infty} \frac{\gamma_{\mathbf{R}}(m)}{m}=3
$$

(v) Se $\operatorname{char} K=2$, então

$$
\lim _{m \rightarrow \infty} \frac{\gamma_{\mathbf{R}}(m)}{m}=\frac{3}{2}
$$

Demonstração: Para a prova de (i), suponhamos char $K \neq 2$. Pelo Lema 4.19, para cada inteiro $m \geq 1$ existem exatamente dois monômios standard de peso $m$, com exceção de $m=1$ e $m=3$, para os quais existe apenas um monômio standard. Assim, para $m \geq 3$, o número de monômios standard com peso menor ou igual a $m$ é

$$
\tilde{\gamma}_{\mathbf{R}}(m)=2 m-2 .
$$


Portanto, $\mathbf{R}$ tem crescimento linear.

Vejamos agora o crescimento de $\mathbf{R}$ quando char $K=2$. Como vimos no Corolário 4.6, os monômios do primeiro tipo e os quadrados dos elementos pivô, $\left\{x_{n-1} v_{n} \mid n \geq 2\right\}$, constituem uma base para $\mathbf{R}$. Observe que, para $n \geq 2$, o monômio do segundo tipo $x_{n-1} v_{n}$ tem peso $\operatorname{wt}\left(x_{n-1} v_{n}\right)=2^{n-1}$. Além disso, para todo $m \geq 1$ existe exatamente um monômio do primeiro tipo com peso igual a $m$ (Lema 4.19). Assim, o número de monômios da base com peso menor ou igual a $m$ é

$$
\tilde{\gamma}_{\mathbf{R}}(m)=m+\left[\log _{2} m\right], \quad m \geq 1
$$

o que prova a afirmação (ii). Em qualquer característica, GKdim $\mathbf{R}=\underline{\operatorname{GKdim}} \mathbf{R}=1$.

Provaremos agora as afirmações (iv) e (v). A função de crescimento $\gamma_{\mathbf{R}}(m)$ dá exatamente o número de monômios standard $v \in \mathbf{R}_{n_{1} n_{2}}$ com grau $n=n_{1}+n_{2} \leq m$. Se $v$ é um monômio standard de multigrau $\left(X_{1}, X_{2}\right)$ e coordenadas peso $\left(Z_{1}, Z_{2}\right)$, o grau $n$ de $v$ satisfaz, pelas relações (4.10),

$$
n=X_{1}+X_{2}=\frac{Z_{1}+2 Z_{2}}{3}+\frac{Z_{1}-Z_{2}}{3}=\frac{2}{3} Z_{1}+\frac{1}{3} Z_{2}
$$

Pelo Teorema 4.18, obtemos as desigualdades

$$
\frac{2}{3} Z_{1}-\left(\frac{\log _{2} Z_{1}+5}{6 Z_{1}}\right) Z_{1} \leq n \leq \frac{2}{3} Z_{1}+\left(\frac{\log _{2} Z_{1}+5}{6 Z_{1}}\right) Z_{1}
$$

Uma vez que $\lim _{Z_{1} \rightarrow \infty} \frac{\log _{2} Z_{1}+5}{6 Z_{1}}=0$, para todo $\varepsilon>0$, temos

$$
\left(\frac{2}{3}-\varepsilon\right) Z_{1} \leq n \leq\left(\frac{2}{3}+\varepsilon\right) Z_{1}
$$

para $Z_{1}$ suficientemente grande. Assim, dado $\varepsilon^{\prime}>0$, existe $N_{\varepsilon^{\prime}} \in \mathbb{N}$ tal que

$$
\left(\frac{3}{2}-\varepsilon^{\prime}\right) n \leq Z_{1} \leq\left(\frac{3}{2}+\varepsilon^{\prime}\right) n, \quad \forall Z_{1} \geq N_{\varepsilon^{\prime}} .
$$

Seja $M_{\varepsilon^{\prime}}$ o número de monômios standard com peso menor que $N_{\varepsilon^{\prime}}$ e fixemos $m \in \mathbb{N}$. Se $v$ é um monômio standard com grau $n \leq m$ e peso $Z_{1}$ suficientemente grande, então

$$
Z_{1} \leq\left(\frac{3}{2}+\varepsilon^{\prime}\right) n \leq\left(\frac{3}{2}+\varepsilon^{\prime}\right) m
$$

Suponhamos char $K \neq 2$. Para cada peso $Z_{1} \geq 4$, existem exatamente dois monômios standard (Lema 4.19). Obtemos, então, uma cota superior para a função de cresci- 
mento:

$$
\gamma_{\mathbf{R}}(m) \leq 2\left(\frac{3}{2}+\varepsilon^{\prime}\right) m+M_{\varepsilon^{\prime}}
$$

Por outro lado, se $v$ é um monômio que satisfaz $N_{\varepsilon^{\prime}} \leq Z_{1} \leq\left(\frac{3}{2}-\varepsilon^{\prime}\right) m$, por (4.13) temos

$$
\left(\frac{3}{2}-\varepsilon^{\prime}\right) n \leq\left(\frac{3}{2}-\varepsilon^{\prime}\right) m
$$

e, portanto, $v$ tem grau $n \leq m$. Obtemos pela afirmação (i) uma cota inferior para a função de crescimento:

$$
\gamma_{\mathbf{R}}(m) \geq 2\left(\frac{3}{2}-\varepsilon^{\prime}\right) m-M_{\varepsilon^{\prime}}
$$

De (4.15) e (4.16), concluímos que $\lim _{m \rightarrow \infty} \frac{\gamma_{\mathbf{R}}(m)}{m}=3$. Supondo agora char $K=2$, da relação (4.14) e da afirmação (ii) segue que

$$
\left(\frac{3}{2}-\varepsilon^{\prime}\right) m+\left[\log _{2} m\right]-M_{\varepsilon^{\prime}} \leq \gamma_{\mathbf{R}}(m) \leq\left(\frac{3}{2}+\varepsilon^{\prime}\right) m+\left[\log _{2} m\right]+M_{\varepsilon^{\prime}}
$$

de onde concluímos que $\lim _{m \rightarrow \infty} \frac{\gamma_{\mathbf{R}}(m)}{m}=\frac{3}{2}$.

Encontrada a dimensão de Gelfand-Kirillov da superálgebra de Lie $\mathbf{R}=\operatorname{Lie}\left(v_{0}, v_{1}\right)$, nosso objetivo agora é determinar o crescimento de sua envoltória associativa $\mathbf{A}=$ $\operatorname{Alg}\left(v_{0}, v_{1}\right)$. Para nosso auxílio, consideraremos a seguir uma superálgebra de Lie um pouco maior $\tilde{\mathbf{R}} \supset \mathbf{R}$ e sua envoltória associativa $\tilde{\mathbf{A}}=\operatorname{Alg}(\tilde{\mathbf{R}}) \supset \mathbf{A}$.

Um monômio quasi-standard de comprimento $n \geq 0$ é um monômio da forma

$$
r_{n-3} x_{n-2}^{\xi_{n-2}} x_{n-1}^{\xi_{n-1}} v_{n}
$$

onde $\xi_{n-2}, \xi_{n-1} \in\{0,1\}$ e $\xi_{n-2}+\xi_{n-1} \leq 1$. Isso significa que um tal monômio contém no máximo uma das variáveis $\left\{x_{n-2}, x_{n-1}\right\}$. O conjunto dos monômios quasi-standard consiste de todos os monômios standard, além de outros dois monômios: $x_{0} v_{1}$ e o monômio falso $x_{0} x_{2} v_{3}$. Introduzimos esses monômios para simplificar o nosso trabalho na demonstração do Teorema 4.22.

Definimos $\tilde{\mathbf{R}}$ como sendo o espaço gerado por todos os monômios quasi-standard. Claramente, $\tilde{\mathbf{R}} \supset \mathbf{R}$.

Lema 4.21 A álgebra $\mathbf{R}=\operatorname{Lie}\left(v_{0}, v_{1}\right)$ está contida numa superálgebra de Lie (restrita) $\tilde{\mathbf{R}} \subset \mathbf{W}(\Lambda)$ cuja base consiste de todos os monômios quasi-standard. 
Demonstração: De forma semelhante à prova do Teorema 4.5, concluímos que todo produto supercomutador de monômios quasi-standard é uma combinação linear de monômios quasi-standard. Isso prova que o espaço vetorial $\tilde{\mathbf{R}}$ definido acima é, na verdade, uma superálgebra de Lie com o produto supercomutador [, ].

No teorema abaixo, consideramos uma subálgebra associativa gerada por monômios quasi-standard.

Teorema 4.22 Seja $\tilde{\mathbf{A}}=\operatorname{Alg}(\tilde{\mathbf{R}}) \subset \operatorname{End}(\Lambda)$. Então

(i) uma base de $\tilde{\mathbf{A}}$ é dada pelos monômios

$\left\{r_{n-3} x_{n-2}^{\xi_{n-2}} x_{n-1}^{\xi_{n-1}} v_{n} v_{n-1}^{\alpha_{n-1}} v_{n-2}^{\alpha_{n-2}} \cdots v_{0}^{\alpha_{0}} \mid \xi_{i}, \alpha_{i} \in\{0,1\} ; \xi_{n-2}+\xi_{n-1} \leq 1+\alpha_{n-1} ; n \geq 0\right\}$, onde $r_{n-3}$ são monômios cauda;

(ii) $\tilde{\mathbf{A}} \supset \mathbf{A}=\operatorname{Alg}\left(v_{0}, v_{1}\right)$;

(iii) monômios com $\xi_{n-1}=0$ são linearmente independentes e pertencem a $\mathbf{A}$.

Demonstração: Considere um monômio $w=r_{n-3} x_{n-2}^{\xi_{n-2}} x_{n-1}^{\xi_{n-1}} v_{n} v_{n-1}^{\alpha_{n-1}} v_{n-2}^{\alpha_{n-2}} \cdots v_{0}^{\alpha_{0}}$, com $\xi_{i}, \alpha_{i} \in\{0,1\}$, satisfazendo a condição $\xi_{n-2}+\xi_{n-1} \leq 1+\alpha_{n-1}$. Observe que a parte inicial de $w$ é um monômio da forma $r_{n-3} x_{n-2}^{\xi_{n-2}} x_{n-1}^{\xi_{n-1}} v_{n} \operatorname{com} \xi_{i} \in\{0,1\}$. Caso $\xi_{n-2}+$ $\xi_{n-1} \leq 1$, tal parte pertence a $\tilde{\mathbf{R}}$, uma vez que se trata de um monômio quasi-standard. Como $v_{i} \in \mathbf{R} \subset \tilde{\mathbf{R}}$ para todo $i \in\{0, \ldots, n-1\}$, w é um produto de elementos de $\tilde{\mathbf{R}}$. Logo, $w \in \tilde{\mathbf{A}}$. Por outro lado, se tivermos $\xi_{n-2}=\xi_{n-1}=1$, devemos ter obrigatoriamente $\alpha_{n-1}=1$. Assim, $w=\left(r_{n-3} x_{n-1} v_{n}\right) \cdot\left(x_{n-2} v_{n-1}\right) \cdot v_{n-2}^{\alpha_{n-2}} \cdots v_{0}^{\alpha_{0}}$ é um produto de elementos de $\tilde{\mathbf{R}}$ e, portanto, pertence a $\tilde{\mathbf{A}}$. A independência linear segue dos mesmos argumentos utilizados em [36, demonstração do Teorema 4.1].

Estamos trabalhando com produtos de monômios quasi-standard. Podemos reordenálos através de argumentos como em PBW, onde é fixada uma ordem total, obedecendo ao comprimento $n$ desses monômios. Nesse processo, produtos (e potências) de monômios de mesmo comprimento desaparecem, uma vez que

$$
\left(r_{n-1} v_{n}\right) \cdot\left(r_{n-1}^{\prime} v_{n}\right)= \pm r_{n-1}^{\prime \prime} v_{n}^{2}= \pm r_{n-1}^{\prime \prime} x_{n+1} v_{n+2}
$$


Obtemos, então, produtos de elementos quasi-standard, escritos em ordem decrescente de comprimento. Obtemos, por exemplo, o produto

$$
\left(r_{n-1} v_{n}\right) \cdot\left(r_{n-2} v_{n-1}\right) \cdots v_{0}
$$

onde há um único monômio quasi-standard de comprimento $n$, enquanto os demais, de comprimentos $n-1, \ldots, 0$, podem ou não aparecer.

Podemos mover todas as letras de Grassmann em (4.17) para a esquerda. De fato, seja $x_{i}$ uma variável de Grassmann num monômio quasi-standard $r_{j-1} v_{j}(i<j)$. Os monômios quasi-standard que o antecedem em (4.17) têm comprimentos maiores que $j$ e, portanto, maiores que $i$. Logo, $x_{i}$ supercomuta com as cabeças $v_{k}$ correspondentes $(k>i)$. No caso em que $r_{n-2} v_{n-1}$ não aparece em (4.17), o fator senior $r_{n-1} v_{n}$ é quasi-standard e, portanto, contém no máximo uma das variáveis $\left\{x_{n-2}, x_{n-1}\right\}$. Visto que não é possível obter alguma dessas variáveis através de produtos com monômios de comprimentos menores, obtemos a condição $\xi_{n-2}+\xi_{n-1} \leq 1$, onde $\alpha_{n-1}=0$. Já no caso em que $r_{n-2} v_{n-1}$ aparece em (4.17), ou seja, no caso em que $\alpha_{n-1}=1$, as variáveis $\left\{x_{n-2}, x_{n-1}\right\}$ podem aparecer livremente. Provamos, portanto, a primeira afirmação.

A segunda afirmação é trivial, uma vez que $\tilde{\mathbf{A}}=\operatorname{Alg}(\tilde{\mathbf{R}}), \mathbf{A}=\operatorname{Alg}(\mathbf{R})$ e $\tilde{\mathbf{R}} \supset \mathbf{R}$. A terceira afirmação decorre do fato de que $r_{n-2} v_{n}, v_{n-1}, \ldots, v_{0} \in \mathbf{R} \subset \mathbf{A}$.

Decorre das estimativas obtidas no Lema 4.15 que o peso de um monômio standard é sempre um inteiro positivo. Podemos generalizar essa afirmação para monômios quasi-standard.

Lema 4.23 Seja $w$ um monômio quasi-standard. Então $\operatorname{wt}(w) \geq 1$.

Demonstração: Com efeito, se $w=r_{n-1} v_{n}$ é um monômio quasi-standard, temos

$$
\mathrm{wt}(w) \geq 2^{n}-2^{n-1}-\cdots-2^{0}=1 .
$$

O próximo lema nos dá estimativas para pesos e superpesos de monômios da base de $\tilde{\mathbf{A}}$ obtida no Teorema 4.22 .

Lema 4.24 Seja $w=r_{n-3} x_{n-2}^{\xi_{n-2}} x_{n-1}^{\xi_{n-1}} v_{n} v_{n-1}^{\alpha_{n-1}} v_{n-2}^{\alpha_{n-2}} \cdots v_{0}^{\alpha_{0}}$ um monômio pertencente a $\tilde{\mathbf{A}}$, com $n \geq 1$, nas mesmas condições do Teorema 4.22. O peso e o superpeso de $w$ 
satisfazem as desigualdades

$$
2^{n-2}<\operatorname{wt}(w)<2^{n+1}, \quad|\operatorname{swt}(w)| \leq n .
$$

Demonstração: Para $n \in\{1,2\}$, essas estimativas podem ser verificadas diretamente. Suponhamos $n \geq 3$. Provaremos primeiro as desigualdades referentes à função peso wt. Se $w$ contém no máximo uma das variáveis de Grassmann $\left\{x_{n-2}, x_{n-1}\right\}$, temos

$2^{n-2}+1=-\left(2^{0}+\cdots+2^{n-3}\right)-2^{n-1}+2^{n} \leq \operatorname{wt}(w) \leq 2^{n}+2^{n-1}+\cdots+2^{0}=2^{n+1}-1$

e, portanto, $2^{n-2}<\operatorname{wt}(w)<2^{n+1}$. No caso em que $\xi_{n-2}=\xi_{n-1}=1, w$ deve necessariamente conter o fator $v_{n-1}$ em sua composição. Assim,

$2^{n-1}+1=-\left(2^{0}+\cdots+2^{n-1}\right)+2^{n}+2^{n-1} \leq \operatorname{wt}(w) \leq 2^{n}+2^{n-1}+\cdots+2^{0}=2^{n+1}-1$

e novamente temos $2^{n-2}<\operatorname{wt}(w)<2^{n+1}$, como queríamos.

Para limitarmos o superpeso, vamos supor primeiramente que $n$ seja par, com $n=2 k$. Uma vez que $-k+1 \leq \operatorname{swt}\left(r_{n-3}\right) \leq k-1, \operatorname{swt}\left(v_{2 k}\right)=1$ e $-k \leq \operatorname{swt}\left(v_{2 k-1}^{\alpha_{2 k-1}} \cdots v_{0}^{\alpha_{0}}\right) \leq k$, temos

$$
-2 k+2 \leq \operatorname{swt}(w) \leq 2 k
$$

No caso em que $n$ é ímpar, com $n=2 k+1$, temos $-k \leq \operatorname{swt}\left(r_{n-3}\right) \leq k-1, \operatorname{swt}\left(v_{2 k+1}\right)=$ $-1,-k \leq \operatorname{swt}\left(v_{2 k}^{\alpha_{2 k}} \cdots v_{0}^{\alpha_{0}}\right) \leq k+1$ e, portanto,

$$
-2 k-1 \leq \operatorname{swt}(w) \leq 2 k-1
$$

Em ambos os casos, vale a limitação $|\operatorname{swt}(w)| \leq n$.

Com as estimativas encontradas no Lema 4.24, obtemos informações a respeito da representação geométrica dos monômios de $\mathbf{A}=\operatorname{Alg}\left(v_{0}, v_{1}\right)$ no plano $\mathbb{R}^{2}$ em termos das coordenadas peso $\left(Z_{1}, Z_{2}\right)$.

Teorema 4.25 O conjunto dos pontos do plano associados aos monômios de $\mathbf{A}=$ $\operatorname{Alg}\left(v_{0}, v_{1}\right)$ é limitado por duas curvas logarítmicas em termos das coordenadas peso $\mathrm{Wt}(w)=\left(Z_{1}, Z_{2}\right)$ :

$$
\left|Z_{2}\right|<\log _{2} Z_{1}+2
$$


Demonstração: Seja $w=r_{n-3} x_{n-2}^{\xi_{n-2}} x_{n-1}^{\xi_{n-1}} v_{n} v_{n-1}^{\alpha_{n-1}} v_{n-2}^{\alpha_{n-2}} \cdots v_{0}^{\alpha_{0}}$ um monômio pertencente a $\mathbf{A}=\operatorname{Alg}\left(v_{0}, v_{1}\right) \subset \tilde{\mathbf{A}}$. Para $n=0$, a verificação é imediata. Suponhamos então $n \geq 1$. Pelo Lema 4.24, valem as desigualdades

$$
2^{n-2}<Z_{1} \quad \text { e } \quad\left|Z_{2}\right| \leq n,
$$

de onde concluímos que $\left|Z_{2}\right|<\log _{2} Z_{1}+2$.

Com as limitações encontradas no Lema 4.24, podemos estimar o crescimento da álgebra $\mathbf{A}=\operatorname{Alg}\left(v_{0}, v_{1}\right)$ e calcular sua dimensão de Gelfand-Kirillov.

Teorema 4.26 Seja $\mathbf{A}=\operatorname{Alg}\left(v_{0}, v_{1}\right)$. Temos GKdim $\mathbf{A}=\underline{G K d i m} \mathbf{A}=2$.

Demonstração: Fixemos um número natural $m \geq 2$ e façamos $n=\left[\log _{2} m\right]-1 \geq 0$. Consideremos monômios $w \in \tilde{\mathbf{A}}$ como no Teorema 4.22 com $\xi_{n-1}=0$. Então, tais monômios são da forma $w=r_{n-2} v_{n} v_{n-1}^{\alpha_{n-1}} v_{n-2}^{\alpha_{n-2}} \cdots v_{0}^{\alpha_{0}}$ e pertencem a A. Pelo Lema 4.24, $\operatorname{wt}(w)<2^{n+1} \leq m$. O número desses monômios constitui uma cota inferior para o crescimento:

$$
\tilde{\gamma}_{\mathbf{A}}(m) \geq 2^{n-1} \cdot 2^{n}=2^{2 n-1} \geq 2^{2 \log _{2} m-5}=2^{\log _{2} m^{2}-5}=\frac{1}{32} m^{2}
$$

Fixemos novamente um número natural $m$ e façamos $n=\left[\log _{2} m\right]+3 \geq 3$. Considere agora um monômio qualquer $w=r_{j-3} x_{j-2}^{\xi_{j-2}} x_{j-1}^{\xi_{j-1}} v_{j} v_{j-1}^{\alpha_{j-1}} v_{j-2}^{\alpha_{j-2}} \cdots v_{0}^{\alpha_{0}}$ pertencente à base de $\tilde{\mathbf{A}}$ determinada no Teorema 4.22. Se $j>n$, então $\operatorname{wt}(w)>2^{j-2}>2^{n-2}>m$. Assim, para que tenhamos $\operatorname{wt}(w) \leq m$, é necessário que se tenha $j \leq n$. Então, o número desses monômios $w$ com $j \leq n$ nos dá uma cota superior para a função $\tilde{\gamma}_{\mathbf{A}}(m)$.

Nessas condições, para $j=0$, temos apenas o monômio $w=v_{0}$. Para $j=1$, temos quatro monômios: $v_{1}, v_{1} v_{0}, x_{0} v_{1}, x_{0} v_{1} v_{0}$. Suponhamos então $j \geq 2$. Nesse caso, temos $2^{j-2}$ possibilidades para $r_{j-3}, 7$ possibilidades de escolha para os coeficientes $\xi_{j-2}, \xi_{j-1}, \alpha_{j-1}$ satisfazendo $\xi_{j-2}+\xi_{j-1} \leq 1+\alpha_{j-1}$, e $2^{j-1}$ possibilidades de escolha para os coeficientes $\alpha_{j-2}, \ldots, \alpha_{0}$. Temos, então, $2^{j-2} \cdot 7 \cdot 2^{j-1}=7 \cdot 2^{2 j-3}$ monômios para $j \geq 2$. Portanto,

$$
\begin{aligned}
\tilde{\gamma}_{\mathbf{A}}(m) & \leq 1+4+\sum_{j=2}^{n} 7 \cdot 2^{2 j-3}=5+7 \cdot 2 \frac{4^{n-1}-1}{4-1}=\frac{1}{3}+\frac{14}{3} \cdot 4^{n-1}<5 \cdot 2^{2 n-2} \\
& \leq 5 \cdot 2^{2 \log _{2} m+4} \leq 80 m^{2} .
\end{aligned}
$$


Segue dessas desigualdades e de (4.18) que GKdim $\mathbf{A}=\underline{\operatorname{GKdim}} \mathbf{A}=2$.

Corolário 4.27 Existem constantes $c_{1}, c_{2}, c_{3}, c_{4}>0$ tais que, para todo $m \geq 1$ :

(i) $c_{1} m^{2} \leq \tilde{\gamma}_{\mathbf{A}}(m) \leq c_{2} m^{2}$;

(ii) $c_{3} m^{2} \leq \gamma_{\mathbf{A}}(m) \leq c_{4} m^{2}$.

Demonstração: Pela demonstração do Teorema 4.26, $c_{1}=\frac{1}{32}$ e $c_{2}=80$ satisfazem $c_{1} m^{2} \leq \tilde{\gamma}_{\mathbf{A}}(m) \leq c_{2} m^{2}$, o que prova (i).

Para a prova da afirmação (ii), observemos que se $u$ pertence a uma base monomial de $\mathbf{A} \subset \tilde{\mathbf{A}}$ e tem peso menor ou igual a $m$, então o seu grau em relação a $\left\{v_{0}, v_{1}\right\}$ também não excede $m$. Logo, $\tilde{\gamma}_{\mathbf{A}}(m) \leq \gamma_{\mathbf{A}}(m)$. Tomando $c_{3}=c_{1}=\frac{1}{32}$, temos $c_{3} m^{2} \leq \gamma_{\mathbf{A}}(m)$. Por outro lado, se $u$ é um monômio pertencente a uma base monomial de $\mathbf{A} \subset \tilde{\mathbf{A}}$ e seu grau em relação a $\left\{v_{0}, v_{1}\right\}$ é menor ou igual a $m$, então seu peso não excede $2 m$. Logo, $\gamma_{\mathbf{A}}(m) \leq \tilde{\gamma}_{\mathbf{A}}(2 m) \leq c_{4} m^{2}$, onde $c_{4}=4 c_{2}>0$.

\subsection{Funções geradoras de $\mathrm{R}$}

Seja $A=\oplus_{n, n \in \mathbb{Z}} A_{n m}$ uma álgebra $\mathbb{Z}^{2}$-graduada, que tem uma $\mathbb{Z}$-graduação induzida: $A=\oplus_{n \in \mathbb{Z}} A_{n}$, onde $A_{n}=\oplus_{m+k=n} A_{m k}$. Definimos as respectivas funções geradoras (ou funções de Hilbert):

$$
\begin{gathered}
\mathcal{H}\left(A, t_{1}, t_{2}\right)=\sum_{n, m} \operatorname{dim} A_{n m} t_{1}^{n} t_{2}^{m} ; \\
\mathcal{H}(A, t)=\sum_{n} \operatorname{dim} A_{n} t^{n}=\mathcal{H}(A, t, t) .
\end{gathered}
$$

Analogamente, definem-se funções geradoras para conjuntos e espaços vetoriais graduados. A superálgebra de Lie $\mathbf{R}=\operatorname{Lie}\left(v_{0}, v_{1}\right)$ é $\mathbb{Z}^{2}$-graduada pelo multigrau, como vimos no Lema 4.9. A fim de obtermos propriedades para a função geradora $\mathcal{H}\left(\mathbf{R}, t_{1}, t_{2}\right)$, provaremos os resultados a seguir.

Lema 4.28 Seja $\mathbf{R}=\operatorname{Lie}\left(v_{0}, v_{1}\right)$. Se $T_{n}^{k}$ é o conjunto dos monômios standard de comprimento $n \geq 4$ e tipo $k \in\{1,2\}$, então

$$
T_{n+1}^{k}=\left\{1, x_{0}\right\} \cdot \tau\left(T_{n}^{k}\right) .
$$


Demonstração: Segue imediatamente da estrutura dos monômios standard.

Provaremos a seguir que a $\mathbb{Z}^{2}$-graduação de $\mathbf{R}$ pelo multigrau em relação aos geradores $\left\{v_{0}, v_{1}\right\}$ é uma graduação fina.

Teorema 4.29 As componentes da $\mathbb{Z}^{2}$-graduação de $\mathbf{R}=\operatorname{Lie}\left(v_{0}, v_{1}\right)$ obtida no Lema 4.9 são no máximo unidimensionais.

Demonstração: Provaremos este resultado por contradição. Suponhamos que existam dois monômios standard distintos $u, v$ com o mesmo multigrau $\operatorname{Gr}(u)=\operatorname{Gr}(v)=$ $\left(X_{1}, X_{2}\right)$ (e, consequentemente, com o mesmo vetor peso $\left.\mathrm{Wt}(u)=\mathrm{Wt}(v)=\left(Z_{1}, Z_{2}\right)\right)$. Pela tabela (4.11), observamos que dois monômios standard distintos não podem possuir o mesmo multigrau se ambos tiverem comprimento menor ou igual a 5. Então, sem perda de generalidade, podemos supor que $u$ tem comprimento maior ou igual a 6. Pelo Lema 4.17, temos $\operatorname{wt}(u)>2^{4}$. Uma vez que $\operatorname{wt}(v)=\operatorname{wt}(u)$, o comprimento de $v$ não pode ser inferior a 5 . Logo, os comprimentos de $u$ e $v$ são ambos maiores ou iguais a 5. Podemos supor que o menor desses comprimentos é minimal sob a condição de termos $u \neq v$ com o mesmo multigrau. Assim, pelo Lema 4.28, temos

$$
u=x_{0}^{\alpha_{0}} \tau(\tilde{u}), \quad v=x_{0}^{\beta_{0}} \tau(\tilde{v}) ; \quad \alpha_{0}, \beta_{0} \in\{0,1\}
$$

onde $\tilde{u}, \tilde{v}$ são monômios standard cujos comprimentos são inferiores aos de $u$ e $v$, respectivamente, diferindo destes em uma unidade. Sejam $\operatorname{Gr}(\tilde{u})=\left(n_{1}, n_{2}\right)$ e $\operatorname{Gr}(\tilde{v})=$ $\left(m_{1}, m_{2}\right)$. Através do Lema 4.14, chegamos à seguinte relação:

$$
A^{T} \cdot\left(\begin{array}{c}
n_{1} \\
n_{2}
\end{array}\right)-\left(\begin{array}{c}
\alpha_{0} \\
0
\end{array}\right)=A^{T} \cdot\left(\begin{array}{c}
m_{1} \\
m_{2}
\end{array}\right)-\left(\begin{array}{c}
\beta_{0} \\
0
\end{array}\right)=\left(\begin{array}{c}
X_{1} \\
X_{2}
\end{array}\right),
$$

onde $A=\left(\begin{array}{ll}0 & 1 \\ 2 & 1\end{array}\right)$. Temos então

$$
\begin{aligned}
\left(\begin{array}{c}
n_{1}-m_{1} \\
n_{2}-m_{2}
\end{array}\right) & =\left(A^{T}\right)^{-1}\left(\begin{array}{c}
\alpha_{0}-\beta_{0} \\
0
\end{array}\right) \\
& =\frac{1}{2}\left(\begin{array}{cc}
-1 & 2 \\
1 & 0
\end{array}\right) \cdot\left(\begin{array}{c}
\alpha_{0}-\beta_{0} \\
0
\end{array}\right) \\
& =\frac{1}{2}\left(\begin{array}{c}
-\left(\alpha_{0}-\beta_{0}\right) \\
\alpha_{0}-\beta_{0}
\end{array}\right) .
\end{aligned}
$$

Como $\alpha_{0}, \beta_{0} \in\{0,1\}$, temos necessariamente $\alpha_{0}-\beta_{0} \in\{-1,0,1\}$. Se ocorresse $\alpha_{0}-$ 
$\beta_{0}= \pm 1$, o número $n_{1}-m_{1}=-\left(n_{2}-m_{2}\right)$ não seria inteiro. Temos, portanto, $\alpha_{0}-\beta_{0}=0$ e, consequentemente, $\left(n_{1}, n_{2}\right)=\left(m_{1}, m_{2}\right)$, isto é, $\operatorname{Gr}(\tilde{u})=\operatorname{Gr}(\tilde{v})$. Pela minimalidade do exemplo, segue que $\tilde{u}=\tilde{v}$. Uma vez que $\alpha_{0}=\beta_{0}$, temos $u=v$ e chegamos numa contradição, o que prova o resultado.

Uma vez que as componentes homogêneas da $\mathbb{Z}^{2}$-graduação de $\mathbf{R}$ pelo multigrau em relação a $\left\{v_{0}, v_{1}\right\}$ são no máximo unidimensionais, temos o seguinte resultado:

Proposição 4.30 Seja $\mathbf{R}=\operatorname{Lie}\left(v_{0}, v_{1}\right)$ e suponhamos char $K \neq 2$. A função geradora $\mathcal{H}\left(\mathbf{R}, t_{1}, t_{2}\right)$ satisfaz a seguinte relação:

$$
\mathcal{H}\left(\mathbf{R}, t_{1}, t_{2}\right)=t_{1}+t_{1}^{2}+t_{1}^{3} t_{2}+t_{1}^{4} t_{2}-t_{1}^{-1} t_{2}-t_{1}^{-1} t_{2}^{2}+\left(1+t_{1}^{-1}\right) \cdot \mathcal{H}\left(\mathbf{R}, t_{2}, t_{1}^{2} t_{2}\right) .
$$

Demonstração: Usando o Teorema 4.29, vemos que os coeficientes da função geradora $\mathcal{H}\left(\mathbf{R}, t_{1}, t_{2}\right)=\sum_{n, m} \operatorname{dim} \mathbf{R}_{n m} t_{1}^{n} t_{2}^{m}$ são iguais a 0 ou 1 . Vamos denotar por $T$ o conjunto de todos os monômios standard e por $T_{n}$ os monômios standard de comprimento $n$, com $n \geq 0$. Por exemplo, para $n \in\{0,1,2,3,4\}$, temos

$$
\begin{aligned}
T_{0}= & \left\{v_{0}\right\} \\
T_{1}= & \left\{v_{1}\right\} \\
T_{2}= & \left\{v_{2}, x_{0} v_{2}, x_{1} v_{2}\right\} \\
T_{3}= & \left\{v_{3}, x_{0} v_{3}, x_{1} v_{3}, x_{2} v_{3}, x_{0} x_{1} v_{3}\right\} \\
T_{4}= & \left\{v_{4}, x_{0} v_{4}, x_{1} v_{4}, x_{2} v_{4}, x_{3} v_{4}, x_{0} x_{1} v_{4}, x_{0} x_{2} v_{4},\right. \\
& \left.x_{0} x_{3} v_{4}, x_{1} x_{2} v_{4}, x_{1} x_{3} v_{4}, x_{0} x_{1} x_{2} v_{4}, x_{0} x_{1} x_{3} v_{4}\right\}
\end{aligned}
$$

Com o auxílio da lista de multigraus apresentada em (4.11), encontramos as funções geradoras $\mathcal{H}\left(T_{n}, t_{1}, t_{2}\right)$ para $n \leq 4$ :

$$
\begin{aligned}
\mathcal{H}\left(T_{0}, t_{1}, t_{2}\right)= & t_{1} ; \\
\mathcal{H}\left(T_{1}, t_{1}, t_{2}\right)= & t_{2} ; \\
\mathcal{H}\left(T_{2}, t_{1}, t_{2}\right)= & t_{1}^{2}+t_{1} t_{2}+t_{1}^{2} t_{2} ; \\
\mathcal{H}\left(T_{3}, t_{1}, t_{2}\right)= & t_{2}^{2}+t_{1} t_{2}^{2}+t_{1}^{2} t_{2}^{2}+t_{1} t_{2}^{3}+t_{1}^{2} t_{2}^{3} ; \\
\mathcal{H}\left(T_{4}, t_{1}, t_{2}\right)= & t_{1}^{3} t_{2}+t_{1}^{4} t_{2}+t_{1}^{3} t_{2}^{2}+t_{1}^{4} t_{2}^{2}+t_{1}^{3} t_{2}^{3}+t_{1}^{4} t_{2}^{3} \\
& +t_{1}^{3} t_{2}^{4}+t_{1}^{4} t_{2}^{4}+t_{1}^{5} t_{2}^{4}+t_{1}^{6} t_{2}^{4}+t_{1}^{5} t_{2}^{5}+t_{1}^{6} t_{2}^{5} .
\end{aligned}
$$

Uma vez que os monômios standard $T$ constituem uma base para $\mathbf{R}=\operatorname{Lie}\left(v_{0}, v_{1}\right)$ 
(Teorema 4.5), temos $\mathcal{H}\left(\mathbf{R}, t_{1}, t_{2}\right)=\mathcal{H}\left(T, t_{1}, t_{2}\right)$. Pelo Lema 4.28 , vale a relação

$$
T_{n+1}=\left\{1, x_{0}\right\} \cdot \tau\left(T_{n}\right), \quad n \geq 4
$$

Portanto, temos

$$
T \backslash\left(T_{0} \cup T_{1} \cup T_{2} \cup T_{3} \cup T_{4}\right)=\left\{1, x_{0}\right\} \cdot \tau\left(T \backslash\left(T_{0} \cup T_{1} \cup T_{2} \cup T_{3}\right)\right)
$$

Observando que $\operatorname{Gr}\left(x_{0}\right)=-\operatorname{Gr}\left(v_{0}\right)=(-1,0)$, temos

$$
\mathcal{H}\left(\left\{x_{0}\right\}, t_{1}, t_{2}\right)=t_{1}^{-1} \quad \text { e } \quad \mathcal{H}\left(\left\{1, x_{0}\right\}, t_{1}, t_{2}\right)=1+t_{1}^{-1} .
$$

Podemos, portanto, computar a função geradora

$$
\begin{aligned}
\mathcal{H}\left(\mathbf{R}, t_{1}, t_{2}\right)= & \mathcal{H}\left(T_{0} \cup T_{1} \cup T_{2} \cup T_{3} \cup T_{4}, t_{1}, t_{2}\right) \\
& +\left(1+t_{1}^{-1}\right) \cdot \mathcal{H}\left(\tau\left(T \backslash\left(T_{0} \cup T_{1} \cup T_{2} \cup T_{3}\right)\right), t_{1}, t_{2}\right) \\
= & \mathcal{H}\left(T_{0} \cup T_{1} \cup T_{2} \cup T_{3} \cup T_{4}, t_{1}, t_{2}\right) \\
& +\left.\left(1+t_{1}^{-1}\right) \cdot\left(\mathcal{H}\left(\mathbf{R}, t_{1}, t_{2}\right)-\mathcal{H}\left(T_{0} \cup T_{1} \cup T_{2} \cup T_{3}, t_{1}, t_{2}\right)\right)\right|_{t_{1}:=t_{2}, t_{2}:=t_{1}^{2} t_{2}} \\
= & t_{1}+t_{2}+t_{1}^{2}+t_{1} t_{2}+t_{2}^{2}+t_{1}^{2} t_{2}+t_{1} t_{2}^{2}+t_{1}^{3} t_{2}+t_{1}^{2} t_{2}^{2}+t_{1} t_{2}^{3}+t_{1}^{4} t_{2}+t_{1}^{3} t_{2}^{2} \\
& +t_{1}^{2} t_{2}^{3}+t_{1}^{4} t_{2}^{2}+t_{1}^{3} t_{2}^{3}+t_{1}^{4} t_{2}^{3}+t_{1}^{3} t_{2}^{4}+t_{1}^{4} t_{2}^{4}+t_{1}^{5} t_{2}^{4}+t_{1}^{6} t_{2}^{4}+t_{1}^{5} t_{2}^{5}+t_{1}^{6} t_{2}^{5} \\
& +\left(1+t_{1}^{-1}\right) \cdot \mathcal{H}\left(\mathbf{R}, t_{2}, t_{1}^{2} t_{2}\right)-t_{2}-t_{1}^{2} t_{2}-t_{1}^{2} t_{2}^{2}-t_{2}^{2}-t_{1}^{2} t_{2}^{3}-t_{1}^{4} t_{2}^{2}-t_{1}^{4} t_{2}^{4} \\
& -t_{1}^{6} t_{2}^{5}-t_{1}^{6} t_{2}^{4}-t_{1}^{4} t_{2}^{3}-t_{1}^{-1} t_{2}-t_{1} t_{2}-t_{1} t_{2}^{2}-t_{1}^{-1} t_{2}^{2}-t_{1} t_{2}^{3}-t_{1}^{3} t_{2}^{2}-t_{1}^{3} t_{2}^{4} \\
& -t_{1}^{5} t_{2}^{5}-t_{1}^{5} t_{2}^{4}-t_{1}^{3} t_{2}^{3} \\
= & t_{1}+t_{1}^{2}+t_{1}^{3} t_{2}+t_{1}^{4} t_{2}-t_{1}^{-1} t_{2}-t_{1}^{-1} t_{2}^{2}+\left(1+t_{1}^{-1}\right) \cdot \mathcal{H}\left(\mathbf{R}, t_{2}, t_{1}^{2} t_{2}\right) .
\end{aligned}
$$

\subsection{Ad-nilpotência dos elementos homogêneos da superálgebra de Lie $\mathbf{R}=\mathbf{R}_{\overline{0}} \oplus \mathbf{R}_{\overline{1}}$}

Para a construção do nosso exemplo $\mathbf{R}$, desejamos obter uma propriedade análoga à periodicidade dos grupos de Grigorchuk e Gupta-Sidki. Para os casos em que char $K=0$ ou char $K=2$, a álgebra associativa $\mathbf{A}=\operatorname{Alg}\left(v_{0}, v_{1}\right)$ não é nil, conforme veremos no Lema 4.31. Contudo, mostraremos no Teorema 4.32 que, para uma característica arbitrária, $\mathbf{R}$ tem uma propriedade semelhante à periodicidade dos grupos mencionados: 
todo elemento homogêneo na $\mathbb{Z}_{2}$-graduação de $\mathbf{R}$ é ad-nilpotente.

Lema 4.31 Se char $K=0$ ou char $K=2$, a álgebra associativa $\mathbf{A}=\operatorname{Alg}\left(v_{0}, v_{1}\right)$ não é nil.

Demonstração: Seja char $K=2$. Para $n \geq 0$ e $0 \neq \alpha \in K$, consideremos o elemento não nulo $v=v_{n}+\alpha x_{n} v_{n+1} \in \mathbf{R}$. Como estamos em característica 2, o quadrado de $v$ pertence a $\mathbf{R}=\operatorname{Lie}\left(v_{0}, v_{1}\right)$. Assim,

$$
\begin{aligned}
v^{2} & =\left(v_{n}+\alpha x_{n} v_{n+1}\right)^{2}=v_{n}^{2}+\left[v_{n}, \alpha x_{n} v_{n+1}\right] \\
& =x_{n+1} v_{n+2}+\alpha v_{n+1} \\
& =\alpha\left(v_{n+1}+\frac{1}{\alpha} x_{n+1} v_{n+2}\right)
\end{aligned}
$$

é um elemento não nulo de $\mathbf{R}$. Observe que $v_{n+1}+\frac{1}{\alpha} x_{n+1} v_{n+2}$ tem uma forma semelhante à de $v$. Assim, temos

$$
\left(v^{2}\right)^{2}=\alpha^{2} \cdot \frac{1}{\alpha}\left(v_{n+2}+\alpha x_{n+2} v_{n+3}\right)=\alpha\left(v_{n+2}+\alpha x_{n+2} v_{n+3}\right),
$$

onde $v_{n+2}+\alpha x_{n+2} v_{n+3}$ também é não nulo e tem forma semelhante a $v$. Repetindo o procedimento de elevar ao quadrado, sempre vamos obter um elemento não nulo, da forma $\alpha^{r}\left(v_{m}+\alpha^{ \pm 1} x_{m} v_{m+1}\right)$. Concluímos assim que a envoltória associativa $\mathbf{A}$ de $\mathbf{R}$ não é nil.

Para o caso em que char $K=0$, o corpo $K$ contém o corpo $\mathbb{Q}$ dos racionais e, portanto, contém o conjunto $\mathbb{Z}$ dos inteiros. Dessa forma, temos

$$
\mathbf{A}=\operatorname{Alg}_{K}\left(v_{0}, v_{1}\right) \supset \operatorname{Alg}_{\mathbb{Z}}\left(v_{0}, v_{1}\right),
$$

onde $\operatorname{Alg}_{\mathbb{Z}}\left(v_{0}, v_{1}\right)$ é o subanel de End $\Lambda$ gerado por $v_{0}$ e $v_{1}$ com coeficientes em $\mathbb{Z}$. Consideremos o homomorfismo sobrejetor

$$
\psi: \operatorname{Alg}_{\mathbb{Z}}\left(v_{0}, v_{1}\right) \rightarrow \operatorname{Alg}_{\mathbb{Z}}\left(v_{0}, v_{1}\right) \otimes_{\mathbb{Z}} \mathbb{F}_{2} \cong \operatorname{Alg}_{\mathbb{F}_{2}}\left(v_{0}, v_{1}\right)
$$

Uma vez que que $\operatorname{Alg}_{\mathbb{F}_{2}}\left(v_{0}, v_{1}\right)$ não é nil, posto que char $\mathbb{F}_{2}=2$, o mesmo acontece para a álgebra $\operatorname{Alg}_{\mathbb{Z}}\left(v_{0}, v_{1}\right)$. Portanto, $\mathbf{A}=\operatorname{Alg}_{K}\left(v_{0}, v_{1}\right) \supset \operatorname{Alg}_{\mathbb{Z}}\left(v_{0}, v_{1}\right)$ não é nil.

O teorema abaixo nos dá uma propriedade análoga à periodicidade dos grupos de Grigorchuk e Gupta-Sidki. 
Teorema 4.32 Seja $\mathbf{R}=\operatorname{Lie}\left(v_{0}, v_{1}\right)=\mathbf{R}_{\overline{0}} \oplus \mathbf{R}_{\overline{1}}$. Para todo $a \in \mathbf{R}_{\bar{n}}, \bar{n} \in\{\overline{0}, \overline{1}\}$, o operador $\operatorname{ad}(a)$ é nilpotente.

Demonstração: Suponhamos primeiramente que $a \in \mathbf{R}_{\overline{1}}$. Nesse caso, temos $(\operatorname{ad}(a))^{2}=\operatorname{ad}\left(a^{2}\right)$ e a nilpotência de $\operatorname{ad}(a)$ pode ser provada a partir da nilpotência de $\operatorname{ad}\left(a^{2}\right)$, onde $a^{2}=\frac{1}{2}[a, a] \in \mathbf{R}_{\overline{0}}$ se char $K \neq 2$ e, se char $K=2$, também temos $a^{2} \in \mathbf{R}_{\overline{0}}$ devido à aplicação quadrática prevista nos axiomas de uma superálgebra de Lie. Logo, podemos reduzir a demonstração deste resultado para o caso em que $a \in \mathbf{R}_{\overline{0}}$. Observe que todo monômio standard par contém pelo menos uma variável de Grassmann, uma vez que os elementos pivô $v_{n}$ são todos ímpares.

Provaremos uma afirmação mais geral. Fixado um número $N>0$, consideremos o conjunto $V$ formado por todos os monômios quasi-standard de comprimento menor ou igual a $N$ que contêm no mínimo uma variável de Grassmann. Afirmamos que a álgebra associativa $\operatorname{Alg}(V) \subset$ End $\Lambda$ é nilpotente.

Seja $w$ um produto não nulo de $M$ elementos $w_{i} \in V$. Decorre do Lema 4.23 que $\operatorname{wt}(w) \geq M$, posto que o peso de cada fator $w_{i}$ satisfaz $\operatorname{wt}\left(w_{i}\right) \geq 1$. Faremos algumas modificações no produto. Vamos mover para a esquerda e ordenar as variáveis de Grassmann, sem alterar a ordem das cabeças. Observe que uma variável de Grassmann $x_{j}$ pode desaparecer ao ser comutada com uma cabeça apropriada $v_{k}$ com $k \leq j$. Vejamos os seguintes exemplos:

$$
\begin{aligned}
& x_{2} v_{5} \cdot x_{5} v_{7}=-x_{2} x_{5} \cdot v_{5} v_{7}+x_{2} \cdot v_{7} ; \\
& x_{3} v_{4} \cdot x_{5} v_{7}=-x_{3} x_{5} \cdot v_{4} v_{7} ; \\
& x_{2} v_{3} \cdot x_{5} v_{7}=-x_{2} x_{5} \cdot v_{3} v_{7}+x_{2} x_{3} x_{4} \cdot v_{7} .
\end{aligned}
$$

Sendo assim, o produto pode ser escrito como uma combinação linear de monômios da forma

$$
u=x_{i_{1}} \cdots x_{i_{n_{1}}} \cdot v_{k_{1}} \cdots v_{k_{n_{2}}}, \quad i_{l}<N, \quad k_{l} \leq N .
$$

Seja $n$ o número de variáveis de Grassmann e seja $m$ o número de cabeças do produto acima, respectivamente. Como $x_{i_{l}} \in\left\{x_{0}, \ldots, x_{N-1}\right\}$ e o produto $u$ é não nulo, o número $n$ de letras de Grassmann distintas acima não excede $N$. Além disso, o número de variáveis de Grassmann no produto original $w$ é maior ou igual ao número de cabeças, e essa propriedade é mantida por transformações como em (4.20). Então $m \leq n \leq N$. 
Estimando o peso do monômio resultante $u$, obtemos

$$
M \leq \operatorname{wt}(w)=\operatorname{wt}(u) \leq m \cdot \operatorname{wt}\left(v_{N}\right) \leq m \cdot 2^{N} \leq n \cdot 2^{N} \leq N \cdot 2^{N} .
$$

Assim, $\operatorname{Alg}(V)^{N_{1}}=0$, onde $N_{1}=N \cdot 2^{N}+1$.

Consideremos agora um elemento par $a \in \mathbf{R}_{\overline{0}}$ qualquer. Sendo assim, $a$ é combinação linear finita de monômios standard pares, os quais contêm pelo menos uma letra de Grassmann. Então, $a \in V$ e, portanto, $a^{N_{1}}=0$. Fazendo $N_{2}=2 N_{1}-1$, para qualquer $b \in \mathbf{R}$ temos

$$
\begin{aligned}
(\operatorname{ad}(a))^{N_{2}}(b) & =\left[a^{N_{2}}, b\right]=\left(l_{a}-r_{a}\right)^{N_{2}}(b)=\sum_{i+j=N_{2}}\left(\begin{array}{c}
N_{2} \\
i
\end{array}\right)(-1)^{j} l_{a}^{i} r_{a}^{j}(b) \\
& =\sum_{i+j=N_{2}}\left(\begin{array}{c}
N_{2} \\
i
\end{array}\right)(-1)^{j} a^{i} b a^{j}=0 .
\end{aligned}
$$

Desse modo, concluímos que ad(a) é nil.

Segue do teorema acima que todo elemento homogêneo na $\mathbb{Z}^{2}$-graduação de $\mathbf{R}$ pelo multigrau em relação a $\left\{v_{0}, v_{1}\right\}$ é ad-nilpotente.

Corolário 4.33 Considere a $\mathbb{Z}^{2}$-graduação $\mathbf{R}=\underset{n_{1}, n_{2} \geq 0}{\oplus} \mathbf{R}_{n_{1} n_{2}}$ apresentada no Lema 4.9. Se a $\in \mathbf{R}_{n_{1} n_{2}}, n_{1}, n_{2} \geq 0$, é um elemento homogêneo dessa graduação, então ad( $(a)$ é nil.

Demonstração: Seja $a \in \mathbf{R}_{n_{1} n_{2}} \operatorname{com} n_{1}, n_{2} \geq 0$. Se $n_{1}+n_{2}$ é par, então $a \in \mathbf{R}_{\overline{0}}$. Se $n_{1}+n_{2}$ é ímpar, temos $a \in \mathbf{R}_{\overline{1}}$. Em qualquer caso, $a$ é homogêneo da $\mathbb{Z}_{2}$-graduação $\mathbf{R}=\mathbf{R}_{\overline{0}} \oplus \mathbf{R}_{\overline{1}}$. Pelo Teorema 4.32, concluímos que ad( $\left.a\right)$ é nil.

Observe que esse resultado é válido para qualquer característica, inclusive zero. A superálgebra de Lie $\mathbf{R}$ construída no nosso trabalho é um exemplo de superálgebra com uma $\mathbb{Z}^{2}$-graduação em que as dimensões das componentes homogêneas são equilimitadas (Teorema 4.29) e os elementos homogêneos são ad-nilpotentes (Corolário 4.33). Com isso, obtemos um contra-exemplo para uma possível extensão do Teorema de Martinez-Zelmanov para superálgebras de Lie.

Teorema 4.34 $\mathrm{Na}$ decomposição triangular $\mathbf{A}=\mathbf{A}_{+} \oplus \mathbf{A}_{0} \oplus \mathbf{A}_{-}$, as subálgebras $\mathbf{A}_{+}$ e $\mathbf{A}_{-}$são localmente nilpotentes. 
Demonstração: Segue dos argumentos utilizados no artigo [36].

\subsection{Just infinitude da superálgebra de Lie $\mathbf{R}$}

No Teorema 4.5 e no Corolário 4.6, mostramos que a superálgebra de $\operatorname{Lie} \mathbf{R}=\operatorname{Lie}\left(v_{0}, v_{1}\right)$ tem dimensão infinita, qualquer que seja a característica do corpo $K$. Contudo, a princípio, se tomarmos quocientes dessa álgebra por ideais bilaterais, podemos obter álgebras de dimensão finita.

Uma $K$-álgebra $A$ é dita just infinite dimensional, ou simplesmente just infinite, se $\operatorname{dim}_{K} A=\infty$ e cada ideal não nulo de $A$ tem codimensão finita. O teorema a seguir mostra que a superálgebra de Lie $\mathbf{R}=\operatorname{Lie}\left(v_{0}, v_{1}\right)$ tem essa propriedade.

Teorema 4.35 A superálgebra de Lie $\mathbf{R}=\operatorname{Lie}\left(v_{0}, v_{1}\right)$ é uma álgebra just infinite.

Demonstração: No Teorema 4.5, exibimos uma base de $\mathbf{R}$ composta por infinitos monômios standard, mostrando que $\operatorname{dim}_{K} \mathbf{R}=\infty$. Consideremos agora $J \triangleleft \mathbf{R}$ um ideal bilateral não nulo de $\mathbf{R}$ e seja $0 \neq w \in J$.

Nosso objetivo agora é encontrar um elemento $v \neq 0$ do ideal $J \triangleleft \mathbf{R}$ tal que, escrito como combinação linear dos monômios standard com coeficientes não nulos, ao menos um desses monômios não contenha letras de Grassmann. Uma vez que $w \in \mathbf{R}$, podemos escrevê-lo como combinação linear finita de monômios standard com coeficientes não nulos. Dentre esses monômios, destacamos os de maior peso, que são no máximo dois, digamos $w_{1}=r_{N-2} v_{N}$ e/ou $w_{2}=r_{N-2} x_{N} v_{N+1}$ (Teorema 4.19). Assim, escrevemos

$$
w=\tilde{w}+\alpha r_{N-2} v_{N}+\beta r_{N-2} x_{N} v_{N+1},
$$

onde pelo menos um dos coeficientes $\{\alpha, \beta\}$ é não nulo e $\tilde{w}$ é uma combinação linear finita de monômios standard com peso inferior a $\operatorname{wt}\left(r_{N-2} v_{N}\right)=\operatorname{wt}\left(r_{N-2} x_{N} v_{N+1}\right)$.

Se $\alpha \neq 0$, multiplicamos $w$ à esquerda sucessivamente por cada elemento pivô $v_{i}$ tal que $x_{i}$ compõe a cauda $r_{N-2}$, eliminando todas as letras de Grassmann de $\alpha r_{N-2} v_{N}$. Obtemos então um elemento não nulo

$$
v=w^{\prime}+\alpha v_{N}+\beta x_{N} v_{N+1} \in J
$$


onde $w^{\prime}$ é combinação linear de monômios standard com peso inferior a $\operatorname{wt}\left(v_{N}\right)$. O termo $\alpha v_{N}(0 \neq \alpha \in K)$ não contém letras de Grassmann, como desejávamos.

Caso tenhamos $\alpha=0$, o coeficiente $\beta$ será não nulo. Multiplicando $w$ à esquerda sucessivamente por cada elemento pivô $v_{i}$ tal que $x_{i}$ compõe a cauda $r_{N-2}$, e posteriormente por $v_{N}$, eliminamos todas as letras de Grassmann de $\beta r_{N-2} x_{N} v_{N+1}$. Obtemos assim um elemento não nulo

$$
v=w^{\prime \prime}+\beta v_{N+1} \in J
$$

onde $w^{\prime \prime}$ é combinação linear de monômios standard com peso inferior a $\operatorname{wt}\left(v_{N+1}\right)$. O termo $\beta v_{N+1}(0 \neq \beta \in K)$ não contém letras de Grassmann, como desejávamos.

Uma vez obtido o elemento $0 \neq v \in J$, seja $m$ o maior comprimento dentre os monômios standard que aparecem na combinação linear, e seja $I$ o conjunto dos índices $i \in$ $\{0, \ldots, m\}$ para os quais essa combinação contém um termo da forma $\lambda_{i} v_{i}, 0 \neq \lambda_{i} \in K$ (sem letras de Grassmann). Multiplicando $v$ à direita pelo elemento $x_{0} \cdots x_{m} v_{m+2}$, anulamos todos os termos que contêm alguma letra de Grassmann e obtemos

$$
\left[v, x_{0} \cdots x_{m} v_{m+2}\right]=\sum_{i \in I} \pm \lambda_{i} x_{0} \cdots \widehat{x_{i}} \cdots x_{m} v_{m+2}, \quad \lambda_{i} \neq 0
$$

Observe que as variáveis de Grassmann comuns a todos os termos de (4.21) são exatamente as letras $x_{i}$ tais que $i \in \bar{I}=\{0, \ldots, m\} \backslash I$. Multiplicando (4.21) à esquerda sucessivamente por elementos pivô $v_{i} \operatorname{com} i \in \bar{I}$ em ordem crescente, eliminamos todas essas variáveis de Grassmann comuns e, fazendo $I=\left\{i_{1}, \ldots, i_{k}\right\}$ com $i_{1}<\ldots<i_{k}$, obtemos

$$
\sum_{j=1}^{k} \pm \lambda_{i_{j}} x_{i_{1}} \cdots \widehat{x_{i_{j}}} \cdots x_{i_{k}} v_{m+2} \in J, \quad \lambda_{i_{j}} \neq 0
$$

Agora multiplicamos (4.22) à esquerda sucessivamente pelos elementos pivô $v_{i_{1}}, v_{i_{2}}, \ldots, v_{i_{k-1}}$, resultando num elemento do tipo

$$
v^{\prime} \pm \lambda_{i_{k}} v_{m+2} \in J
$$

onde $v^{\prime}$ é uma combinação linear (possivelmente nula) de monômios standard de comprimento $m+2$ que contêm pelo menos uma letra de Grassmann. Multiplicando (4.23) por $x_{0} \cdots x_{m+2} v_{m+4}$, anulamos $v^{\prime}$ e obtemos

$$
\left[v^{\prime} \pm \lambda_{i_{k}} v_{m+2}, x_{0} \cdots x_{m+2} v_{m+4}\right]= \pm \lambda_{i_{k}} x_{0} \cdots x_{m+1} v_{m+4}
$$


Multiplicando (4.24) à esquerda sucessivamente por $v_{0}, \ldots, v_{m+1}$, eliminamos todas as letras de Grassmann e concluímos que $v_{m+4} \in J$.

Observe que $-\left[v_{m+3}^{2}, v_{m+4}\right]=v_{m+5}$ também pertence ao ideal $J$. Usando o item (iv) do Lema 4.1, uma simples indução nos faz concluir que $v_{n} \in J$ para qualquer $n \geq m+4$.

Vamos mostrar que todo monômio standard de comprimento $n \geq m+7$ pertence a $J$. Com efeito, se $n \geq m+7$, temos

$$
\left[v_{n-2}, x_{0} \cdots x_{n-3} v_{n-1}\right]= \pm x_{0} \cdots x_{n-2} v_{n} \in J
$$

Podemos eliminar qualquer fator $x_{i}$ fazendo o produto comutador do elemento acima com $v_{i}, i=0, \ldots, n-2$. Assim, $J$ contém todos os monômios standard do primeiro tipo de comprimento $n \geq m+7$.

Agora vamos mostrar que os monômios standard do segundo tipo de comprimento $n \geq m+7$ também pertencem a $J$. Suponhamos primeiramente char $K \neq 2$. Se $n \geq m+7$ é par, temos

$$
\frac{1}{2}\left[v_{0}, v_{n-2}\right]=x_{0} \cdots x_{n-3} x_{n-1} v_{n} \in J
$$

Fazendo o produto comutador desse elemento com $v_{i}, i=0, \ldots, n-2$, podemos eliminar qualquer fator $x_{i}$ acima. No caso em que $n$ é ímpar, temos

$$
\begin{gathered}
-\left[x_{0} v_{n-4}, v_{n-4}, v_{n-3}\right]=x_{0} v_{n-2} \in J, \\
\frac{1}{2}\left[v_{1}, x_{0} v_{n-2}\right]=x_{0} \cdots x_{n-3} x_{n-1} v_{n} \in J
\end{gathered}
$$

Podemos eliminar qualquer fator $x_{i}$ fazendo o produto comutador do elemento acima com $v_{i}, i=0, \ldots, n-3$. Dessa forma, concluímos que todos os monômios standard do segundo tipo de comprimento $n \geq m+7$ pertencem ao ideal $J$.

No caso em que char $K=2$, a operação $x \mapsto x^{2}$, onde $x$ é ímpar, pertence aos axiomas de superálgebra de Lie. Então, $v_{n}^{2}=x_{n+1} v_{n+2} \in J$ para todo $n \geq m+4$.

Em qualquer característica, provamos então que $J$ contém todos os monômios standard de comprimento maior ou igual a $m+7$ pertencentes à base de $\mathbf{R}$. Portanto, a dimensão da álgebra quociente $\mathbf{R} / J$ é finita e não excede o número de monômios standard de comprimento menor ou igual a $m+6$. 


\section{Capítulo 5}

\section{A álgebra de Lie restrita $R$}

Neste capítulo, estudaremos as álgebras de Lie do Exemplo 2. Seja $K$ um corpo de característica $p>0$. Consideremos a álgebra de polinômios truncada $\Delta=K\left[x_{i} \mid i \geq\right.$ $0] /\left(x_{i}^{p}\right)$. Dentre as derivações dessa álgebra, destacamos os seguintes elementos:

$$
v_{i}=\partial_{x_{i}}+x_{i}^{p-1} x_{i+1}^{p-1}\left(\partial_{x_{i+2}}+x_{i+2}^{p-1} x_{i+3}^{p-1}\left(\partial_{x_{i+4}}+x_{i+4}^{p-1} x_{i+5}^{p-1}\left(\partial_{x_{i+6}}+\cdots\right)\right)\right), \quad i \geq 0 .
$$

A esses elementos $v_{i} \in$ Der $\Delta$ damos o nome de elementos pivô. Eles podem ser escritos na forma recursiva

$$
v_{i}=\partial_{x_{i}}+x_{i}^{p-1} x_{i+1}^{p-1} v_{i+2}, \quad i \geq 0 .
$$

Definimos a álgebra de Lie $\mathbf{L}=\operatorname{Lie}\left(v_{0}, v_{1}\right) \subset \operatorname{Der} \Delta$.

\subsection{Relações principais}

Para cada $i \geq 0$, definimos $l_{x_{i}} \in$ End $\Delta$ por $l_{x_{i}}(p)=x_{i} \cdot p$. Dessa forma, cada variável $x_{i}$ pode ser vista como um endomorfismo da álgebra $\Delta$. Portanto, $\left\{x_{i}, \partial_{x_{i}} \mid i \geq 0\right\} \subset$ End $\Delta$. Valem as seguintes relações:

$$
\begin{gathered}
x_{i} x_{j}=x_{j} x_{i}, \quad \partial_{x_{i}} \partial_{x_{j}}=\partial_{x_{j}} \partial_{x_{i}}, \quad \partial_{x_{i}} x_{j}=x_{j} \partial_{x_{i}}, \quad i \neq j ; \\
x_{i}^{p}=0, \quad\left(\partial_{x_{i}}\right)^{p}=0, \quad i \geq 0 .
\end{gathered}
$$

Também é válida a seguinte relação não trivial: 


$$
\partial_{x_{i}} x_{i}-x_{i} \partial_{x_{i}}=1, \quad i \geq 0 .
$$

Os elementos pivô $v_{i}$ agem sobre as variáveis $x_{i}$ da seguinte maneira:

$$
v_{n}\left(x_{k}\right)= \begin{cases}0, & k<n ; \\ 1, & k=n ; \\ 0, & k=n+2 l-1, \quad l>0 \\ x_{n}^{p-1} x_{n+1}^{p-1} \cdots x_{k-2}^{p-1} x_{k-1}^{p-1}, & k=n+2 l, \quad l>0 .\end{cases}
$$

Definimos as aplicações shift $\tau: \Delta \rightarrow \Delta$ e $\tau: \mathbf{W}(\Delta) \rightarrow \mathbf{W}(\Delta)$ por

$$
\tau\left(x_{i}\right)=x_{i+1}, \quad \tau\left(\partial_{x_{i}}\right)=\partial_{x_{i+1}}, \quad i \geq 0
$$

É fácil ver que $\tau$ é um endomorfismo de $\mathbf{W}(\Delta)$. Recordamos que os comutadores aqui são normados à direita: $[a, b, c]=[a,[b, c]]$. Usamos também a notação $\left[(a)_{k}, b\right]:=$ $[a, \ldots, a, b]$, onde $a$ aparece $k$ vezes no produto de Lie. Obtemos no resultado a seguir algumas relações básicas entre os elementos pivô.

Lema 5.1 São válidas as seguintes relações, para $i \geq 0$ :

(i) $\left[v_{i}, v_{i+1}\right]=x_{i}^{p-1} x_{i+1}^{p-2} v_{i+2}$;

(ii) $\left[v_{i}, v_{i+2}\right]=0$;

(iii) $\left[\left(v_{i}\right)_{2}, v_{i+1}\right]=-x_{i}^{p-2} x_{i+1}^{p-2} v_{i+2}$;

(iv) $\left[\left(v_{i}\right)_{p}, v_{i+1}\right]=-x_{i+1}^{p-2} v_{i+2}$;

(v) $\left[\left(v_{i+1}\right)_{p-2},\left(v_{i}\right)_{p}, v_{i+1}\right]=-v_{i+2}$.

Demonstração: As igualdades

$$
\begin{aligned}
{\left[v_{i}, v_{i+1}\right] } & =\left[\partial_{x_{i}}+x_{i}^{p-1} x_{i+1}^{p-1} v_{i+2}, \partial_{x_{i+1}}+x_{i+1}^{p-1} x_{i+2}^{p-1} v_{i+3}\right] \\
& =\left[x_{i+1}^{p-1}, \partial_{x_{i+1}}\right] x_{i}^{p-1} v_{i+2}=-(p-1) x_{i+1}^{p-2} x_{i}^{p-1} v_{i+2} \\
& =x_{i}^{p-1} x_{i+1}^{p-2} v_{i+2}
\end{aligned}
$$

provam a afirmação (i). Além disso, temos

$$
\left[v_{i}, v_{i+2}\right]=\left[\partial_{x_{i}}+x_{i}^{p-1} x_{i+1}^{p-1} v_{i+2}, v_{i+2}\right]=0
$$


o que prova a afirmação (ii). Para a prova da afirmação (iii), observemos que

$$
\left[\left(v_{i}\right)_{2}, v_{i+1}\right]=\left[\partial_{x_{i}}+x_{i}^{p-1} x_{i+1}^{p-1} v_{i+2}, x_{i}^{p-1} x_{i+1}^{p-2} v_{i+2}\right]=-x_{i}^{p-2} x_{i+1}^{p-2} v_{i+2}
$$

Uma simples indução nos faz concluir que

$$
\left[\left(v_{i}\right)_{p}, v_{i+1}\right]=(-1)^{p-1}(p-1) ! x_{i+1}^{p-2} v_{i+2}=(-1)^{p} x_{i+1}^{p-2} v_{i+2}=-x_{i+1}^{p-2} v_{i+2},
$$

tornando válida a afirmação (iv). De forma semelhante, obtemos

$$
\left[\left(v_{i+1}\right)_{p-2},\left(v_{i}\right)_{p}, v_{i+1}\right]=(-1)^{p-1}(p-1) ! v_{i+2}=-v_{i+2},
$$

provando assim a afirmação (v).

O lema a seguir mostra como calcular o produto comutador entre dois elementos pivô quaisquer, generalizando os itens (i) e (ii) do lema acima.

Lema 5.2 Para quaisquer inteiros $i, k \geq 0$, temos:

(i) se $k$ é par, então $\left[v_{i}, v_{i+k}\right]=0$;

(ii) se $k$ é ímpar, então $\left[v_{i}, v_{i+k}\right]=x_{i}^{p-1} x_{i+1}^{p-1} \cdots x_{i+k-1}^{p-1} x_{i+k}^{p-2} v_{i+k+1}$.

Demonstração: Se $k$ é par, temos

$$
\left[v_{i}, v_{i+k}\right]=\left[\partial_{x_{i}}+x_{i}^{p-1} x_{i+1}^{p-1} \partial_{x_{i+2}}+\cdots+x_{i}^{p-1} \cdots x_{i+k-1}^{p-1} v_{i+k}, v_{i+k}\right]=0
$$

provando a afirmação (i). Por outro lado, se $k$ é ímpar, pelo item (i) do Lema 5.1 temos

$$
\begin{aligned}
{\left[v_{i}, v_{i+k}\right] } & =\left[\partial_{x_{i}}+x_{i}^{p-1} x_{i+1}^{p-1} \partial_{i+2}+\cdots+x_{i}^{p-1} \cdots x_{i+k-2}^{p-1} v_{i+k-1}, v_{i+k}\right] \\
& =x_{i}^{p-1} \cdots x_{i+k-2}^{p-1} x_{i+k-1}^{p-1} x_{i+k}^{p-2} v_{i+k+1}
\end{aligned}
$$

tornando válida a afirmação (ii).

$\mathrm{Na}$ álgebra associativa End $\Delta$, podemos calcular as potências de grau $p$ dos elementos pivô $v_{i}$ como no lema abaixo:

Lema 5.3 Para todo $i \geq 0$, vale

$$
v_{i}^{p}=-x_{i+1}^{p-1} v_{i+2} .
$$


Demonstração: Na álgebra de Lie End $\Delta^{(-)}$, a correspondência $x \mapsto x^{p}$ define uma $p$-aplicação. Dessa forma, para $x, y \in \operatorname{End} \Delta$, temos

$$
(x+y)^{p}=x^{p}+y^{p}+(\operatorname{ad} y)^{p-1}(x)+\sum_{i=2}^{p-1} s_{i}(x, y),
$$

onde os comutadores em $s_{i}(x, y)$ contêm $i$ letras $x$ e $p-i$ letras $y$. Segue então que

$$
\begin{aligned}
v_{i}^{p}= & \left(\partial_{x_{i}}+x_{i}^{p-1} x_{i+1}^{p-1} v_{i+2}\right)^{p} \\
= & \left(\partial_{x_{i}}\right)^{p}+\left(x_{i}^{p-1} x_{i+1}^{p-1} v_{i+2}\right)^{p}+\left(\operatorname{ad} \partial_{x_{i}}\right)^{p-1}\left(x_{i}^{p-1} x_{i+1}^{p-1} v_{i+2}\right) \\
& +\sum_{i=2}^{p-1} s_{i}\left(x_{i}^{p-1} x_{i+1}^{p-1} v_{2}, \partial_{x_{i}}\right) .
\end{aligned}
$$

Para $2 \leq i \leq p-1$, o termo $s_{i}\left(x_{i}^{p-1} x_{i+1}^{p-1} v_{2}, \partial_{x_{i}}\right)$ envolve comutadores em que o elemento $x_{i}^{p-1} x_{i+1}^{p-1} v_{2}$ aparece em grau maior ou igual a 2 e a derivação $\partial_{x_{i}}$ aparece em grau menor ou igual a $p-2$. Isso implica que $s_{i}\left(x_{i}^{p-1} x_{i+1}^{p-1} v_{2}, \partial_{x_{i}}\right)=0$ para todo $i \in\{2, \ldots, p-1\}$. Segue de $(5.1)$ que $v_{i}^{p}=\left(\operatorname{ad} \partial_{x_{i}}\right)^{p-1}\left(x_{i}^{p-1} x_{i+1}^{p-1} v_{i+2}\right)=-x_{i+1}^{p-1} v_{i+2}$.

As potências de grau $p$ na álgebra End $\Delta$ definem uma $p$-aplicação na álgebra de Lie End $^{(-)} \Delta$. Podemos, então, definir $\mathbf{R}=\operatorname{Lie}_{p}\left(v_{0}, v_{1}\right)$, a álgebra de Lie restrita gerada por $v_{0}$ e $v_{1}$. Observe que $\mathbf{L} \subset \mathbf{R}$.

Consideremos as seguintes álgebras de Lie restritas geradas por dois elementos: $L_{i}=$ $\operatorname{Lie}_{p}\left(v_{i}, v_{i+1}\right), i \geq 0$. Em particular, temos $L_{0}=\mathbf{R}$.

\section{Corolário 5.4 Temos}

(i) $v_{i} \in \mathbf{R}, i \geq 0$;

(ii) $\tau^{i}: \mathbf{R} \rightarrow L_{i}$ é um isomorfismo para todo $i \geq 0$;

(iii) $\mathbf{R}$ tem dimensão infinita;

(iv) existe uma imersão de autossimilaridade:

$$
\mathbf{R} \hookrightarrow\left\langle\partial_{x_{0}}\right\rangle_{K} \curlywedge\left(K\left[x_{0}\right] /\left(x_{0}^{p}\right) \otimes \tau(\mathbf{R})\right)
$$




\subsection{Bases de $\mathrm{L}$ e $\mathrm{R}$}

Nosso objetivo agora é encontrar uma base para a álgebra de $\operatorname{Lie} \mathbf{L}=\operatorname{Lie}\left(v_{0}, v_{1}\right)$. Para simplificar a notação, denotaremos por $r_{n}$ um monômio cauda:

$$
r_{n}=x_{0}^{\xi_{0}} \cdots x_{n}^{\xi_{n}} ; \quad \xi_{0}, \ldots, \xi_{n} \in\{0, \ldots, p-1\}, n \geq 0
$$

Quando necessário, denotaremos outros monômios cauda por $r_{n}^{\prime}, r_{n}^{\prime \prime}$, etc. De agora em diante, os monômios $v_{0}, v_{1}$ e todos os monômios do tipo

$$
r_{n-2} x_{n-1}^{\xi_{n-1}} v_{n}, \quad 0 \leq \xi_{n-1} \leq p-2, \quad n \geq 2
$$

serão referidos como monômios standard do primeiro tipo. Já os monômios da forma

$$
x_{n-1}^{p-1} v_{n}, \quad n \geq 2,
$$

serão designados como monômios standard do segundo tipo.

Lema 5.5 Todos os monômios standard do primeiro tipo

$$
\left\{v_{0}, v_{1}\right\} \cup\left\{r_{n-2} x_{n-1}^{\xi_{n-1}} v_{n} \mid 0 \leq \xi_{n-1} \leq p-2, n \geq 2\right\}
$$

pertencem à álgebra de Lie $\mathbf{L}=\operatorname{Lie}\left(v_{0}, v_{1}\right)$.

Demonstração: Primeiramente, provaremos por indução sobre $n$ que todo elemento pivô $v_{n}, n \geq 0$, pertence a $\mathbf{L}=\operatorname{Lie}\left(v_{0}, v_{1}\right)$. Obviamente, os monômios $v_{0}$ e $v_{1}$ pertencem a $\mathbf{L}$. Tomemos agora $n \geq 2$ e suponhamos que $v_{i} \in \mathbf{L}$ para todo $0 \leq i \leq n-1$. Então, o item (v) do Lema 5.1 nos garante que

$$
v_{n}=-\left[\left(v_{n-1}\right)_{p-2},\left(v_{n-2}\right)_{p}, v_{n-1}\right] \in \mathbf{L} .
$$

Provaremos agora que todo elemento da forma $r_{n-1} v_{n}$, com $n \geq 2$, pertence a $\mathbf{L}$. Suponhamos primeiramente que $n$ seja par. Pelo Lema 5.2, temos

$$
\left[v_{0}, v_{n-1}\right]=x_{0}^{p-1} \cdots x_{n-2}^{p-1} x_{n-1}^{p-2} v_{n} \in \mathbf{L}
$$

Multiplicando o elemento acima por elementos pivô $v_{i}$, podemos eliminar alguns dos fatores $x_{i}$, onde $0 \leq i \leq n-1$. 
Vejamos agora o caso em que $n \geq 2$ é ímpar. Uma vez que $n-1$ é par, já sabemos que $x_{0}^{p-1} v_{n-1}$ pertence a $\mathbf{L}$. Dessa forma, o Lema 5.2 nos garante que

$$
\left[v_{1}, x_{0}^{p-1} v_{n-1}\right]=x_{0}^{p-1} \cdots x_{n-2}^{p-1} x_{n-1}^{p-2} v_{n} \in \mathbf{L}
$$

Podemos eliminar alguns dos fatores $x_{i}$ multiplicando o elemento acima por elementos pivô $v_{i}$, onde $0 \leq i \leq n-1$.

Dado um monômio $v=r_{n-1} v_{n}$, o número inteiro $n \geq 0$ é denominado o comprimento de $v$. Provaremos a seguir que os monômios apresentados no Lema 5.5 constituem uma base para a álgebra de Lie $\mathbf{L}=\operatorname{Lie}\left(v_{0}, v_{1}\right)$.

Teorema 5.6 O conjunto dos monômios standard do primeiro tipo

$$
\left\{v_{0}, v_{1}\right\} \cup\left\{r_{n-2} x_{n-1}^{\xi_{n-1}} v_{n} \mid 0 \leq \xi_{n-1} \leq p-2, n \geq 2\right\}
$$

constitui uma base para a álgebra de Lie $\mathbf{L}=\operatorname{Lie}\left(v_{0}, v_{1}\right)$.

Demonstração: Provamos no Lema 5.5 que todos os monômios standard do primeiro tipo pertencem a L. Para mostrar que eles geram L como espaço vetorial, é suficiente provar que qualquer produto comutador entre dois desses monômios é combinação linear de monômios standard do primeiro tipo. Os produtos que consideraremos a seguir podem eventualmente resultar em elementos com alguns termos nulos, caso algum fator $x_{i}$ apareça em grau maior ou igual a $p$. Por simplicidade, vamos supor que cada fator $x_{i}$ aparece no máximo em grau $p-1$.

1) Consideremos produtos entre monômios de mesmo comprimento $n$. Observe que

$$
\left[r_{n-1} v_{n}, r_{n-1}^{\prime} v_{n}\right]=r_{n-1}^{\prime \prime}\left[v_{n}, v_{n}\right]=0
$$

é uma combinação linear de elementos de $B$.

2) Consideremos agora produtos entre monômios de comprimentos distintos $n<m$, com $n \equiv m(\bmod 2)$. Nesse caso, temos

$$
v_{n}=\partial_{x_{n}}+x_{n}^{p-1} x_{n+1}^{p-1}\left(\partial_{x_{n+2}}+\cdots+x_{m-4}^{p-1} x_{m-3}^{p-1}\left(\partial_{x_{m-2}}+x_{m-2}^{p-1} x_{m-1}^{p-1} v_{m}\right) \cdots\right) .
$$


Assim,

$$
\begin{aligned}
{\left[x_{0}^{\xi_{0}} \cdots x_{n-1}^{\xi_{n-1}} v_{n}, x_{0}^{\eta_{0}} \cdots x_{m-1}^{\eta_{m-1}} v_{m}\right]=} & \eta_{n} x_{0}^{\gamma_{0}} \cdots x_{n-1}^{\gamma_{n-1}} x_{n}^{\eta_{n}-1} x_{n+1}^{\eta_{n+1}} \cdots x_{m-1}^{\eta_{m-1}} v_{m} \\
& +\eta_{n+2} x_{0}^{\gamma_{0}} \cdots x_{n-1}^{\gamma_{n-1}} x_{n}^{p-1} x_{n+1}^{p-1} x_{n+2}^{\eta_{n+2}-1} x_{n+3}^{\eta_{n+3}} \cdots x_{m-1}^{\eta_{m-1}} v_{m} \\
& +\cdots \\
& +\eta_{m-2} x_{0}^{\gamma_{0}} \cdots x_{n-1}^{\gamma_{n-1}} x_{n}^{p-1} \cdots x_{m-3}^{p-1} x_{m-2}^{\eta_{m-2}-1} x_{m-1}^{\eta_{m-1}} v_{m},
\end{aligned}
$$

onde $\gamma_{i}=\xi_{i}+\eta_{i}, i \in\{0, \ldots, n-1\}$, é uma combinação linear de monômios standard do primeiro tipo, visto que $0 \leq \eta_{m-1} \leq p-2$.

3) Por fim, vamos considerar produtos entre monômios de comprimentos distintos $n<m, \operatorname{com} n \equiv m-1(\bmod 2)$. Assim, temos

$$
v_{n}=\partial_{x_{n}}+x_{n}^{p-1} x_{n+1}^{p-1}\left(\partial_{x_{n+2}}+\cdots+x_{m-5}^{p-1} x_{m-4}^{p-1}\left(\partial_{x_{m-3}}+x_{m-3}^{p-1} x_{m-2}^{p-1} v_{m-1}\right) \cdots\right) .
$$

Supondo $\eta_{m-1} \neq 0$, o produto

$$
\begin{aligned}
{\left[x_{0}^{\xi_{0}} \cdots x_{n-1}^{\xi_{n-1}} v_{n}, x_{0}^{\eta_{0}} \cdots x_{m-1}^{\eta_{m-1}} v_{m}\right]=} & \eta_{n} x_{0}^{\gamma_{0}} \cdots x_{n-1}^{\gamma_{n-1}} x_{n}^{\eta_{n}-1} x_{n+1}^{\eta_{n+1}} \cdots x_{m-1}^{\eta_{m-1}} v_{m} \\
& +\eta_{n+2} x_{0}^{\gamma_{0}} \cdots x_{n-1}^{\gamma_{n-1}} x_{n}^{p-1} x_{n+1}^{p-1} x_{n+2}^{\eta_{n+2}-1} x_{n+3}^{\eta_{n+3}} \cdots x_{m-1}^{\eta_{m-1}} v_{m} \\
& +\cdots \\
& +\eta_{m-1} x_{0}^{\gamma_{0}} \cdots x_{n-1}^{\gamma_{n-1}} x_{n}^{p-1} \cdots x_{m-2}^{p-1} x_{m-1}^{\eta_{m-1}-1} v_{m},
\end{aligned}
$$

onde $\gamma_{i}=\xi_{i}+\eta_{i}, i \in\{0, \ldots, n-1\}$, é uma combinação linear de monômios standard do primeiro tipo, posto que $0 \leq \eta_{m-1} \leq p-2$. No caso em que $\eta_{m-1}=0$, o produto

$$
\begin{aligned}
{\left[x_{0}^{\xi_{0}} \cdots x_{n-1}^{\xi_{n-1}} v_{n}, x_{0}^{\eta_{0}} \cdots x_{m-1}^{\eta_{m-1}} v_{m}\right]=} & \eta_{n} x_{0}^{\gamma_{0}} \cdots x_{n-1}^{\gamma_{n-1}} x_{n}^{\eta_{n}-1} x_{n+1}^{\eta_{n+1}} \cdots x_{m-2}^{\eta_{m-2}} v_{m} \\
& +\eta_{n+2} x_{0}^{\gamma_{0}} \cdots x_{n-1}^{\gamma_{n-1}} x_{n}^{p-1} x_{n+1}^{p-1} x_{n+2}^{\eta_{n+2}-1} x_{n+3}^{\eta_{n+3}} \cdots x_{m-2}^{\eta_{m-2}} v_{m} \\
& +\cdots \\
& +\eta_{m-3} x_{0}^{\gamma_{0}} \cdots x_{n-1}^{\gamma_{n-1}} x_{n}^{p-1} \cdots x_{m-4}^{p-1} x_{m-3}^{\eta_{m-3}-1} x_{m-2}^{\eta_{m-2}} v_{m} \\
& +x_{0}^{\gamma_{0}} \cdots x_{n-1}^{\gamma_{n-1}} x_{n}^{p-1} \cdots x_{m-2}^{p-1} x_{m-1}^{p-1} x_{m}^{p-2} v_{m+1},
\end{aligned}
$$

onde $\gamma_{i}=\xi_{i}+\eta_{i}, i \in\{0, \ldots, n-1\}$, também é uma combinação linear de monômios standard do primeiro tipo, visto que $0 \leq \eta_{m-2} \leq p-2$.

Uma vez que os monômios standard do primeiro tipo são linearmente independentes [36], eles constituem uma base para a álgebra de $\operatorname{Lie} \mathbf{L}=\operatorname{Lie}\left(v_{0}, v_{1}\right)$. 
Encontrada uma base para $\mathbf{L}=\operatorname{Lie}\left(v_{0}, v_{1}\right)$, nossa meta agora é obter uma base para a álgebra de Lie restrita $\mathbf{R}=\operatorname{Lie}_{p}\left(v_{0}, v_{1}\right)$, levando em consideração as potências de grau $p$ dos elementos pivô apresentadas no Lema 5.3.

Corolário 5.7 Os monômios standard do primeiro tipo

$$
\left\{v_{0}, v_{1}\right\} \cup\left\{r_{n-2} x_{n-1}^{\xi_{n-1}} v_{n} \mid 0 \leq \xi_{n-1} \leq p-2, n \geq 2\right\}
$$

e os monômios standard do segundo tipo

$$
\left\{x_{n-1}^{p-1} v_{n} \mid n \geq 2\right\}
$$

formam uma base para a álgebra de Lie restrita $\mathbf{R}=\operatorname{Lie}_{p}\left(v_{0}, v_{1}\right)$.

Demonstração: Vimos no Teorema 5.6 que os monômios standard do primeiro tipo constituem uma base $B$ para a subálgebra de Der $\Delta$ gerada por $v_{0}, v_{1}$ considerando apenas o colchete comutador [, ]. Para obter a álgebra de Lie restrita $\mathbf{R}=\operatorname{Lie}_{p}\left(v_{0}, v_{1}\right)=\operatorname{Lie}_{p}(\mathbf{L})$, é suficiente adicionar todas as potências $\left\{w^{\left[p^{n}\right]} \mid w \in B, n \geq 1\right\}$. Essas potências são triviais, a menos que sejam potências de grau $p$ de elementos pivô. Do Lema 5.3, concluímos que o conjunto de monômios $B \cup\left\{x_{n-1}^{p-1} v_{n} \mid n \geq 2\right\}$ é uma base da álgebra de Lie restrita $\mathbf{R}=\operatorname{Lie}_{p}\left(v_{0}, v_{1}\right)$.

\subsection{Funções peso}

A fim de obtermos o crescimento da álgebra de Lie restrita $\mathbf{R}=\operatorname{Lie}_{p}\left(v_{0}, v_{1}\right)$ e de sua envoltória associativa $\mathbf{A}=\operatorname{Alg}(\mathbf{R}) \subset$ End $\Delta$, recorreremos ao uso de uma função peso wt. Suponhamos que os pesos das derivações $\partial_{x_{i}}$ e das variáveis $x_{i}$ satisfazem

$$
\operatorname{wt}\left(\partial_{x_{i}}\right)=-\operatorname{wt}\left(x_{i}\right)=\alpha_{i} \in \mathbb{C}, \quad i \geq 0 .
$$

Queremos que todos os termos na relação de recorrência

$$
v_{i}=\partial_{x_{i}}+x_{i}^{p-1} x_{i+1}^{p-1} v_{i+2}, \quad i \geq 0
$$

tenham o mesmo peso e que, além disso, a função peso seja aditiva em monômios nas 
letras $\left\{x_{i}, \partial_{x_{i}}, v_{i} \mid i \geq 0\right\}$. Dessa forma, para cada $i \geq 0$ temos

$$
\operatorname{wt}\left(v_{i}\right)=\operatorname{wt}\left(\partial_{x_{i}}\right)=\alpha_{i}, \quad \alpha_{i}=-(p-1) \alpha_{i}-(p-1) \alpha_{i+1}+\alpha_{i+2} .
$$

Obtemos a relação de recorrência

$$
\alpha_{i+2}=p \alpha_{i}+(p-1) \alpha_{i+1}, \quad i \geq 0 .
$$

$\mathrm{Na}$ forma matricial,

$$
\left(\begin{array}{c}
\alpha_{i+1} \\
\alpha_{i+2}
\end{array}\right)=A\left(\begin{array}{c}
\alpha_{i} \\
\alpha_{i+1}
\end{array}\right), i \geq 0 ; \text { onde } A=\left(\begin{array}{cc}
0 & 1 \\
p & p-1
\end{array}\right)
$$

Os autovalores da matriz $A$ (raízes do polinômio característico de (5.5)) são $\lambda_{1}=p$ e $\lambda_{2}=-1$. Então, as sequências $\alpha_{i}^{(1)}=p^{i}(i \geq 0)$ e $\alpha_{i}^{(2)}=(-1)^{i}(i \geq 0)$ formam uma base para o espaço de soluções da relação de recorrência (5.5). Dessa forma, podemos introduzir duas funções peso como segue.

Lema 5.8 Temos as seguintes funções peso:

(i) $\operatorname{wt}\left(\partial_{x_{n}}\right)=\operatorname{wt}\left(v_{n}\right)=p^{n}, n \geq 0$ (a função peso);

(ii) $\operatorname{swt}\left(\partial_{x_{n}}\right)=\operatorname{swt}\left(v_{n}\right)=(-1)^{n}, n \geq 0$ (a função superpeso);

(iii) $\operatorname{Wt}\left(v_{n}\right)=\left(\operatorname{wt}\left(v_{n}\right), \operatorname{swt}\left(v_{n}\right)\right)=\left(p^{n},(-1)^{n}\right), n \geq 0$ (a função vetor peso).

Demonstração: Segue dos comentários feitos acima.

De agora em diante, chamaremos simplesmente de monômios quaisquer monômios nas letras $\left\{x_{i}, \partial_{x_{i}}, v_{i} \mid i \geq 0\right\}$, pertencentes à álgebra associativa End $\Delta$.

Lema 5.9 As funções peso estão bem definidas em todos os monômios. Além disso, são aditivas em produtos de monômios, i.e., se a e b são dois monômios desse tipo, então

$$
\mathrm{Wt}(a \cdot b)=\mathrm{Wt}(a)+\mathrm{Wt}(b)
$$

Demonstração: Segue da forma como construímos as funções peso. 


\section{$5.4 \quad \mathbb{Z}^{2}$-graduação de $\mathrm{R}$; coordenadas peso e multi- grau}

Lema 5.10 As álgebras $\mathbf{R}=\operatorname{Lie}_{p}\left(v_{0}, v_{1}\right)$ e $\mathbf{A}=\operatorname{Alg}(\mathbf{R})$ são $\mathbb{Z}^{2}$-graduadas pelo multigrau em relação a $\left\{v_{0}, v_{1}\right\}$ :

$$
\mathbf{R}=\underset{n_{1}, n_{2} \geq 0}{\bigoplus} \mathbf{R}_{n_{1} n_{2}}, \quad \mathbf{A}=\underset{n_{1}, n_{2} \geq 0}{\bigoplus} \mathbf{A}_{n_{1} n_{2}} .
$$

Demonstração: Os vetores peso $\operatorname{Wt}\left(v_{0}\right)=(1,1)$ e $\operatorname{Wt}\left(v_{1}\right)=(p,-1)$ dados no Lema 5.8 são linearmente independentes. Obtemos, então, um reticulado peso:

$$
\Gamma=\mathbb{Z} \mathrm{Wt}\left(v_{0}\right) \oplus \mathbb{Z} \mathrm{Wt}\left(v_{1}\right) \subset \mathbb{R}^{2}
$$

Para $n_{1}, n_{2} \geq 0$, seja $\mathbf{A}_{n_{1} n_{2}} \subset \mathbf{A}$ o subespaço gerado por todos os monômios de graus $n_{1}$ e $n_{2}$ em relação aos geradores $v_{0}$ e $v_{1}$, respectivamente. Pelo Lema 5.9, para todo $v \in \mathbf{A}_{n_{1} n_{2}}$, temos

$$
\mathrm{Wt}(v)=n_{1} \mathrm{Wt}\left(v_{0}\right)+n_{2} \mathrm{Wt}\left(v_{1}\right)=\left(n_{1}+p n_{2}, n_{1}-n_{2}\right) .
$$

Como os vetores $\mathrm{Wt}\left(v_{0}\right)$ e $\mathrm{Wt}\left(v_{1}\right)$ são linearmente independentes, concluímos que elementos pertencentes a componentes $\mathbf{A}_{n_{1} n_{2}}$ distintas são linearmente independentes.

A prova para a álgebra de Lie restrita $\mathbf{R}=\operatorname{Lie}_{p}\left(v_{0}, v_{1}\right)$ é análoga.

Observe que se $v \in \mathbf{A}_{n_{1} n_{2}}$, então $\operatorname{swt}(v)=n_{1} \operatorname{swt}\left(v_{0}\right)+n_{2} \operatorname{swt}\left(v_{1}\right)=n_{1}-n_{2}=0$ se, e somente se, $n_{1}=n_{2}$. A diagonal da $\mathbb{Z}^{2}$-graduação de $\mathbf{A}$ é definida como sendo a soma direta das componentes homogêneas cujos elementos têm superpeso nulo, ou seja, a subálgebra $\oplus \mathbf{A}_{n n}$. Analogamente, a diagonal da $\mathbb{Z}^{2}$-graduação de $\mathbf{R}$ é dada

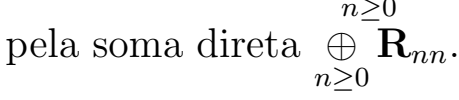

Corolário 5.11 Valem as seguintes afirmações:

(i) As álgebras $\mathbf{R}$ e $\mathbf{A}$ possuem decomposição triangular em somas diretas de três subálgebras:

$$
\mathbf{R}=\mathbf{R}_{+} \oplus \mathbf{R}_{0} \oplus \mathbf{R}_{-}, \quad \mathbf{A}=\mathbf{A}_{+} \oplus \mathbf{A}_{0} \oplus \mathbf{A}_{-}
$$


onde as componentes são geradas por monômios de superpeso positivo, zero e negativo, respectivamente;

(ii) As componentes nulas $\mathbf{R}_{0}$ e $\mathbf{A}_{0}$ coincidem com as diagonais correspondentes $\mathbf{R}_{0}=$ $\underset{n \geq 0}{\oplus} \mathbf{R}_{n n}$ e $\mathbf{A}_{0}=\underset{n \geq 0}{\oplus} \mathbf{A}_{n n}$.

Demonstração: Segue da aditividade da função superpeso.

Dado um monômio não nulo $v \in \mathbf{A}_{n_{1} n_{2}}, n_{1}, n_{2} \geq 0$, definimos o vetor multigrau

$$
\operatorname{Gr}(v)=\left(n_{1}, n_{2}\right) \in \mathbb{Z}^{2} \subset \mathbb{R}^{2} .
$$

Podemos identificá-lo no plano $\mathbb{R}^{2}$ com coordenadas standard $\left(X_{1}, X_{2}\right)$, as quais chamamos de coordenadas multigrau, fazendo $\operatorname{Gr}(v)=\left(n_{1}, n_{2}\right)=\left(X_{1}, X_{2}\right)$.

Seja $\left(X_{1}, X_{2}\right) \in \mathbb{R}^{2}$ um ponto qualquer do plano. Introduzimos também as coordenadas peso $\left(Z_{1}, Z_{2}\right)$ no plano $\mathbb{R}^{2}$ da seguinte maneira:

$$
\left\{\begin{array} { l } 
{ Z _ { 1 } = X _ { 1 } + p X _ { 2 } ; } \\
{ Z _ { 2 } = X _ { 1 } - X _ { 2 } ; }
\end{array} \quad \left\{\begin{array}{l}
X_{1}=\left(Z_{1}+p Z_{2}\right) /(p+1) \\
X_{2}=\left(Z_{1}-Z_{2}\right) /(p+1)
\end{array}\right.\right.
$$

Lema 5.12 Seja $v \in \mathbf{A}$ um monômio, $\operatorname{Gr}(v)=\left(X_{1}, X_{2}\right)$ o seu multigrau, e $\left(Z_{1}, Z_{2}\right)$ as suas coordenadas peso. Então, $\left(Z_{1}, Z_{2}\right)=\mathrm{Wt}(v)=(\operatorname{wt}(v), \operatorname{swt}(v))$.

Demonstração: Através de (5.7), colocamos o peso e o superpeso em função do multigrau, o que nos dá

$$
\operatorname{wt}(v)=n_{1}+p n_{2}=X_{1}+p X_{2}=Z_{1}, \quad \operatorname{swt}(v)=n_{1}-n_{2}=X_{1}-X_{2}=Z_{2} .
$$

Lema 5.13 São válidas as seguintes afirmações:

(i) O multigrau de um elemento pivô $v_{n}$ é dado por

$$
\operatorname{Gr}\left(v_{n}\right)=\frac{1}{p+1}\left(p^{n}+p(-1)^{n}, p^{n}-(-1)^{n}\right), \quad n \geq 0
$$

(ii) Os elementos pivô $v_{n}$ pertencem à união de duas retas no plano: $X_{1}-X_{2}=1$ e $X_{1}-X_{2}=-1$. 
Demonstração: A afirmação (i) segue do fato de que $\mathrm{Wt}\left(v_{n}\right)=\left(p^{n},(-1)^{n}\right)$ e das relações entre as coordenadas (5.8). Para a verificação do item (ii), observemos que para um elemento pivô $v_{n}$ temos $X_{1}-X_{2}=Z_{2}=\operatorname{swt}\left(v_{n}\right)=(-1)^{n}$. Assim, se $n$ é par, $v_{n}$ pertence à reta $X_{1}-X_{2}=1$. Por outro lado, se $n$ é ímpar, $v_{n}$ está localizado na $\operatorname{reta} X_{1}-X_{2}=-1$.

Pela expressão obtida no Lema 5.13 para o multigrau de um elemento pivô, podemos estabelecer uma relação entre os multigraus de $v_{n}$ e $\tau\left(v_{n}\right)=v_{n+1} \cdot \operatorname{Se} \operatorname{Gr}\left(v_{n}\right)=\left(n_{1}, n_{2}\right)$, temos

$$
\begin{aligned}
\operatorname{Gr}\left(v_{n+1}\right) & =\frac{1}{p+1}\left(p^{n+1}+p(-1)^{n+1}, p^{n+1}-(-1)^{n+1}\right) \\
& =\frac{1}{p+1}\left(p\left(p^{n}-(-1)^{n}\right), p^{n}+p(-1)^{n}+(p-1)\left(p^{n}-(-1)^{n}\right)\right) \\
& =\left(p n_{2}, n_{1}+(p-1) n_{2}\right)
\end{aligned}
$$

Podemos generalizar essa relação para um elemento $v \in \mathbf{A}_{n_{1} n_{2}}$ e sua imagem pelo endomorfismo $\tau$ :

Lema 5.14 Seja $0 \neq v \in \mathbf{A}_{n_{1} n_{2}}, n_{1}, n_{2} \geq 0$, e seja $\tau(v)$ sua imagem pelo endomorfismo T. Se $\operatorname{Gr}(\tau(v))=\left(m_{1}, m_{2}\right)$, então

$$
\left(\begin{array}{l}
m_{1} \\
m_{2}
\end{array}\right)=\left(\begin{array}{c}
p n_{2} \\
n_{1}+(p-1) n_{2}
\end{array}\right)=A^{T} \cdot\left(\begin{array}{c}
n_{1} \\
n_{2}
\end{array}\right)
$$

onde $A=\left(\begin{array}{cc}0 & 1 \\ p & p-1\end{array}\right)$ é a matriz definida na relação de recorrência (5.6).

Demonstração: Uma vez que $v \in \mathbf{A}_{n_{1} n_{2}}$, o elemento $v$ é uma combinação linear de produtos de $n_{1}$ fatores de $v_{0}$ e $n_{2}$ fatores de $v_{1}$. Então vale a igualdade

$$
\operatorname{Gr}(v)=n_{1} \operatorname{Gr}\left(v_{0}\right)+n_{2} \operatorname{Gr}\left(v_{1}\right) .
$$

Como $\tau$ é um endomorfismo, $\tau(v)$ é uma combinação linear de produtos de $n_{1}$ fatores de $\tau\left(v_{0}\right)$ e $n_{2}$ fatores de $\tau\left(v_{1}\right)$. Sabendo que $\tau\left(v_{0}\right)=v_{1}$ e $\tau\left(v_{1}\right)=v_{2}$, concluímos pela aditividade da função multigrau que

$$
\operatorname{Gr}(\tau(v))=n_{1} \operatorname{Gr}\left(v_{1}\right)+n_{2} \operatorname{Gr}\left(v_{2}\right)=n_{1}(0,1)+n_{2}(p, p-1)=\left(p n_{2}, n_{1}+(p-1) n_{2}\right) .
$$




\subsection{Estimativas para os pesos}

O lema a seguir nos dá estimativas para os pesos dos monômios standard do primeiro tipo.

Lema 5.15 Se $w=x_{0}^{\xi_{0}} \cdots x_{n-1}^{\xi_{n-1}} v_{n}$ é um monômio standard do primeiro tipo de comprimento $n \geq 1$, então

$$
p^{n-1}+1 \leq \operatorname{wt}(w) \leq p^{n}
$$

Demonstração: Recordemo-nos de que $\operatorname{wt}\left(v_{i}\right)=-\operatorname{wt}\left(x_{i}\right)=p^{i}$ para todo $i \geq 0$. Visto que $\xi_{i} \in\{0, \ldots, p-1\}$ para todo $i \in\{0, \ldots, n-2\}$ e $\xi_{n-1} \in\{0, \ldots, p-2\}$, pela aditividade da função peso temos

$$
\begin{aligned}
p^{n} \geq \operatorname{wt}\left(x_{0}^{\xi_{0}} \cdots x_{n-1}^{\xi_{n-1}} v_{n}\right) & \geq p^{n}-\left((p-1)\left(1+p+\cdots+p^{n-2}\right)+(p-2) p^{n-1}\right) \\
& =p^{n}-\left(p^{n-1}-1+(p-2) p^{n-1}\right) \\
& =p^{n}-\left((p-1) p^{n-1}-1\right) \\
& =p^{n-1}+1 .
\end{aligned}
$$

No lema abaixo, computamos os pesos dos monômios standard do segundo tipo.

Lema 5.16 Se $w=x_{n-1}^{p-1} v_{n}$ é um monômio standard do segundo tipo de comprimento $n \geq 2$, então $\operatorname{wt}(w)=p^{n-1}$.

Demonstração: Basta observarmos que

$$
\operatorname{wt}\left(x_{n-1}^{p-1} v_{n}\right)=\operatorname{wt}\left(v_{n}\right)-(p-1) \operatorname{wt}\left(x_{n-1}\right)=p^{n}-(p-1) p^{n-1}=p^{n-1} .
$$

No próximo lema, obtemos estimativas para os superpesos dos monômios standard do primeiro tipo.

Lema 5.17 Se $w=x_{0}^{\xi_{0}} \cdots x_{n-1}^{\xi_{n-1}} v_{n}$ é um monômio standard do primeiro tipo de comprimento $n \geq 1$, então

$$
-\frac{n+1}{2}(p-1) \leq \operatorname{swt}(w) \leq \frac{n}{2}(p-1)
$$


Demonstração: Suponhamos que o comprimento $n$ de $w$ seja par e façamos $n=2 k$. Levando em conta que $\xi_{i} \in\{0, \ldots, p-1\}$ para todo $i \in\{0, \ldots, n-2\}$ e $\xi_{n-1} \in$ $\{0, \ldots, p-2\}$, e considerando os superpesos

$$
\begin{gathered}
\operatorname{swt}\left(x_{0}\right)=\operatorname{swt}\left(x_{2}\right)=\cdots=\operatorname{swt}\left(x_{2 k-2}\right)=-1, \\
\operatorname{swt}\left(x_{1}\right)=\operatorname{swt}\left(x_{3}\right)=\cdots=\operatorname{swt}\left(x_{2 k-1}\right)=1, \\
\operatorname{swt}\left(v_{n}\right)=1,
\end{gathered}
$$

obtemos as seguintes desigualdades:

$$
-\frac{n}{2}(p-1)+1=-k(p-1)+1 \leq \operatorname{swt}(w) \leq(k-1)(p-1)+p-2+1=\frac{n}{2}(p-1) .
$$

Vejamos agora o caso em que o comprimento $n$ de $w$ é ímpar, com $n=2 k+1$. Uma vez que $\operatorname{swt}\left(v_{2 k+1}\right)=-1$, temos

$$
-\frac{n+1}{2}(p-1)=-k(p-1)-(p-2)-1 \leq \operatorname{swt}(w) \leq k(p-1)-1=\frac{n-1}{2}(p-1)-1 .
$$

Em ambos os casos, valem as desigualdades

$$
-\frac{n+1}{2}(p-1) \leq \operatorname{swt}(w) \leq \frac{n}{2}(p-1)
$$

No lema a seguir, calcularemos os superpesos dos monômios standard do segundo tipo.

Lema 5.18 Se $w=x_{n-1}^{p-1} v_{n}$ é um monômio standard do segundo tipo de comprimento $n \geq 2$, então $\operatorname{swt}(w)=(-1)^{n} p$.

Demonstração: Basta observarmos que swt $\left(x_{n-1}\right)=\operatorname{swt}\left(v_{n}\right)=(-1)^{n}$.

Agora podemos obter estimativas mais gerais para os pesos e superpesos dos monômios standard.

Lema 5.19 Seja $w$ um monômio standard de comprimento $n \geq 0$. Então

$$
p^{n-1} \leq \operatorname{wt}(w) \leq p^{n}, \quad-\frac{n+1}{2} p \leq \operatorname{swt}(w) \leq \frac{n}{2} p+1 .
$$


Demonstração: Se $n \geq 1$, as cotas para $\operatorname{wt}(w)$ decorrem diretamente do Lema 5.15 e do Lema 5.16. Em relação ao superpeso, se $w$ é do primeiro tipo, pelo Lema 5.17 temos

$$
-\frac{n+1}{2} p \leq-\frac{n+1}{2}(p-1) \leq \operatorname{swt}(w) \leq \frac{n}{2}(p-1) \leq \frac{n}{2} p+1 .
$$

Por outro lado, se $w$ é do segundo tipo, segue do Lema 5.18 que

$$
-\frac{n+1}{2} p \leq-p \leq \operatorname{swt}(w) \leq p \leq \frac{n}{2} p+1 \text {. }
$$

No caso específico em que $n=0$, temos $\operatorname{wt}(w)=1=p^{n} \operatorname{e~} \operatorname{swt}(w)=1=\frac{n}{2} p+1$.

As estimativas encontradas no Lema 5.19 nos dão informações referentes à representação geométrica dos monômios standard no plano $\mathbb{R}^{2}$ em termos das coordenadas peso $\left(Z_{1}, Z_{2}\right)$, como mostra o teorema a seguir.

Teorema 5.20 O conjunto dos pontos do plano associados aos monômios standard é limitado por duas curvas logarítmicas em termos das coordenadas peso $\mathrm{Wt}(w)=$ $\left(Z_{1}, Z_{2}\right)$ :

$$
-\frac{p}{2}\left(\log _{p} Z_{1}+2\right) \leq Z_{2} \leq \frac{p}{2}\left(\log _{p} Z_{1}+1\right)+1
$$

Demonstração: Seja $w$ um monômio standard de comprimento $n \geq 0$. Pelo Lema 5.19 , vale a desigualdade $p^{n-1} \leq Z_{1}$, de onde obtemos $n \leq \log _{p} Z_{1}+1$. Pelo mesmo lema, conseguimos cotas para $Z_{2}=\operatorname{swt}(w)$ :

$$
-\frac{p}{2}\left(\log _{p} Z_{1}+2\right) \leq-\frac{n+1}{2} p \leq Z_{2} \leq \frac{n}{2} p+1 \leq \frac{p}{2}\left(\log _{p} Z_{1}+1\right)+1 .
$$

\subsection{Crescimento de $\mathrm{R}$ e $\mathrm{A}$}

A fim de obtermos o crescimento da álgebra de Lie restrita $\mathbf{R}=\operatorname{Lie}_{p}\left(v_{0}, v_{1}\right)$, provaremos o seguinte lema:

Lema 5.21 Seja $m \geq 1$ um inteiro. Valem as seguintes afirmações:

(i) Existe um monômio standard do primeiro tipo com peso igual a $m$ se, e somente se, $m=1$ ou $m \geq p$. Neste caso, $w_{1}$ é único; 
(ii) Existe um monômio standard do segundo tipo $w_{2}$ tal que $\operatorname{wt}\left(w_{2}\right)=m$ se, e somente se, $m=p^{k}$ com $k \geq 1$. Neste caso, $w_{2}$ é único;

(iii) Se $m=p^{k}$ com $k \geq 1$, então $w_{1}=v_{k}$ e $w_{2}=x_{k}^{p-1} v_{k+1}=-v_{k+1}^{p}$.

Demonstração: Para os pesos $m=1$ e $m=p$, temos os monômios do primeiro tipo $v_{0}$ e $v_{1}$, respectivamente. Como consequência do Lema 5.15, temos o fato de que os monômios standard de comprimento $n \geq 2$ têm peso maior ou igual a $p+1$. Fixemos agora um inteiro $n \geq 2$. Pelo Lema 5.15, um monômio standard do primeiro tipo de comprimento $n$ tem peso $\operatorname{wt}\left(x_{0}^{\xi_{0}} \cdots x_{n-1}^{\xi_{n-1}} v_{n}\right) \in\left\{p^{n-1}+1, \ldots, p^{n}\right\}$. Reciprocamente, para cada $m \in\left\{p^{n-1}+1, \ldots, p^{n}\right\}$ existe um único monômio standard do primeiro tipo $w_{1}$ tal que $\operatorname{wt}\left(w_{1}\right)=m$. Com efeito, o inteiro $c=p^{n}-m \in\left\{0,1, \ldots, p^{n}-p^{n-1}-1\right\}$ pode ser escrito de maneira única na forma $c=\xi_{0} \cdot p^{0}+\xi_{1} \cdot p^{1}+\cdots+\xi_{n-2} \cdot p^{n-2}+\xi_{n-1} \cdot p^{n-1}$ com $0 \leq \xi_{0}, \ldots, \xi_{n-2} \leq p-1$ e $0 \leq \xi_{n-1} \leq p-2$. Dessa forma, $w_{1}=x_{0}^{\xi_{0}} \cdots x_{n-1}^{\xi_{n-1}} v_{n}$ é o único monômio standard do primeiro tipo que satisfaz $\operatorname{wt}\left(w_{1}\right)=p^{n}-c=m$. Portanto, para todo $m \geq p+1$ existe um único monômio standard do primeiro tipo com peso igual a $m$. Isso conclui a prova de (i). O item (ii) segue diretamente do Lema 5.16 e a prova de (iii) é imediata.

Agora podemos computar o crescimento da álgebra de Lie restrita $\mathbf{R}=\operatorname{Lie}_{p}\left(v_{0}, v_{1}\right) \mathrm{e}$ encontrar sua dimensão de Gelfand-Kirillov.

Teorema 5.22 Valem as seguintes afirmações:

(i) Considerando a função peso wt, obtemos a seguinte função de crescimento para $\mathbf{R}$ :

$$
\tilde{\gamma}_{\mathbf{R}}(m)=m+\left[\log _{p} m\right]-p+2, \quad m \geq p .
$$

(ii) $\mathrm{GKdim} \mathbf{R}=\underline{\mathrm{GKdim}} \mathbf{R}=1$.

Demonstração: Consideremos $m \geq p$. Pelo item (i) do Lema 5.21, o número de monômios standard do primeiro tipo com peso menor ou igual a $m$ é $m-p+2$. O item (ii) do mesmo lema mostra que o número de monômios standard do segundo tipo com peso igual ou inferior a $m$ é $\left[\log _{p} m\right]$. Logo,

$$
\tilde{\gamma}_{\mathbf{R}}(m)=m+\left[\log _{p} m\right]-p+2, \quad m \geq p .
$$


Portanto, GKdim $\mathbf{R}=\underline{\operatorname{GKdim}} \mathbf{R}=\lim _{m \rightarrow \infty} \frac{\ln \tilde{\gamma}_{\mathbf{R}}(m)}{\ln m}=1$.

Encontrada a dimensão de Gelfand-Kirillov da álgebra de Lie restrita $\mathbf{R}=\operatorname{Lie}_{p}\left(v_{0}, v_{1}\right)$, nosso objetivo agora é determinar o crescimento de sua envoltória associativa $\mathbf{A}=\operatorname{Alg}(\mathbf{R})$. Para nosso auxílio, consideraremos a seguir uma álgebra de Lie um pouco maior $\tilde{\mathbf{R}} \supset \mathbf{R}$ e sua envoltória associativa $\tilde{\mathbf{A}}=\operatorname{Alg}(\tilde{\mathbf{R}}) \supset \mathbf{A}$.

Um monômio quasi-standard de comprimento $n \geq 0$ é um monômio da forma

$$
x_{0}^{\xi_{0}} \cdots x_{n-2}^{\xi_{n-2}} x_{n-1}^{\xi_{n-1}} v_{n}
$$

onde $\xi_{n-2}, \xi_{n-1} \in\{0, \ldots, p-1\}$ e $\xi_{n-2}+\xi_{n-1} \leq 2(p-1)-1$. Isso significa que um tal monômio não pode conter as letras $x_{n-2}, x_{n-1}$ em grau $p-1$ simultaneamente. Observe que todos os monômios standard são, em particular, monômios quasi-standard.

Definimos $\tilde{\mathbf{R}}$ como sendo o subespaço de End $\Delta$ gerado por todos os monômios quasistandard. Claramente, $\tilde{\mathbf{R}} \supset \mathbf{R}$.

Lema 5.23 A álgebra de Lie restrita $\mathbf{R}=\operatorname{Lie}_{p}\left(v_{0}, v_{1}\right)$ está contida em outra álgebra de Lie $\tilde{\mathbf{R}} \subset$ End $\Delta$ cuja base consiste de todos os monômios quasi-standard.

Demonstração: De forma análoga à prova do Teorema 5.6, concluímos que todo produto comutador de monômios quasi-standard é uma combinação linear de monômios quasi-standard. Isso prova que o espaço vetorial $\tilde{\mathbf{R}}$ definido acima é, na verdade, uma álgebra de Lie com o produto comutador $[$,$] .$

No teorema abaixo, consideramos uma álgebra associativa gerada por monômios quasistandard.

Teorema 5.24 Seja $\tilde{\mathbf{A}}=\operatorname{Alg}(\tilde{\mathbf{R}}) \subset$ End $\Delta$. Então

(i) uma base de $\tilde{\mathbf{A}}$ é dada por todos os monômios da forma

$$
x_{0}^{\xi_{0}} \cdots x_{n-2}^{\xi_{n-2}} x_{n-1}^{\xi_{n-1}} v_{n}^{\alpha_{n}} v_{n-1}^{\alpha_{n-1}} v_{n-2}^{\alpha_{n-2}} \cdots v_{0}^{\alpha_{0}}, \quad n \geq 0
$$


onde $0 \leq \xi_{i}, \alpha_{i} \leq p-1$ para todo $i \in\{0, \ldots, n\}, \alpha_{n} \geq 1$ e, no caso específico em que $\alpha_{n}=1$ e $\alpha_{n-1}=0$, adiciona-se a condição $\xi_{n-2}+\xi_{n-1} \leq 2(p-1)-1$;

(ii) $\tilde{\mathbf{A}} \supset \mathbf{A}=\operatorname{Alg}(\mathbf{R})$;

(iii) monômios com $\xi_{n-1} \leq p-2$ são linearmente independentes e pertencem a $\mathbf{A}$.

Demonstração: Considere um monômio $w=r_{n-3} x_{n-2}^{\xi_{n-2}} x_{n-1}^{\xi_{n-1}} v_{n} v_{n-1}^{\alpha_{n-1}} v_{n-2}^{\alpha_{n-2}} \cdots v_{0}^{\alpha_{0}}$, com $\xi_{i}, \alpha_{i} \in\{0,1, \ldots, p-1\}, \alpha_{n} \geq 1$, satisfazendo a condição $\xi_{n-2}+\xi_{n-1} \leq 2(p-1)-1$ caso se tenha $\alpha_{n}=1$ e $\alpha_{n-1}=0$. Observe que a parte inicial de $w$ é um monômio da forma $r_{n-3} x_{n-2}^{\xi_{n-2}} x_{n-1}^{\xi_{n-1}} v_{n}$ com $\xi_{i} \in\{0,1, \ldots, p-1\}$. Caso $\xi_{n-2}+\xi_{n-1} \leq 2(p-1)-1$, tal parte pertence a $\tilde{\mathbf{R}}$, uma vez que se trata de um monômio quasi-standard. Como $v_{i} \in \mathbf{R} \subset \tilde{\mathbf{R}}$ para todo $i \in\{0, \ldots, n\}, w$ é um produto de elementos de $\tilde{\mathbf{R}}$. Logo, $w \in \tilde{\mathbf{A}}$. Por outro lado, se tivermos $\xi_{n-2}=\xi_{n-1}=p-1$, devemos ter obrigatoriamente $\alpha_{n} \geq 2$ ou $\alpha_{n-1} \geq 1$. Temos, então, duas possibilidades: $w=\left(r_{n-3} x_{n-2}^{p-2} x_{n-1}^{p-1} v_{n}\right) \cdot\left(x_{n-2} v_{n}\right)$. $v_{n}^{\alpha_{n}-2} \cdot v_{n-1}^{\alpha_{n-1}} \cdots v_{0}^{\alpha_{0}}\left(\operatorname{caso} \alpha_{n} \geq 2\right)$ ou $w=\left(r_{n-3} x_{n-2}^{p-2} x_{n-1}^{p-1} v_{n}\right) \cdot v_{n}^{\alpha_{n}-1} \cdot\left(x_{n-2} v_{n-1}\right) \cdot v_{n-1}^{\alpha_{n-1}-1}$. $v_{n-2}^{\alpha_{n-2}} \cdots v_{0}^{\alpha_{0}}\left(\right.$ caso $\left.\alpha_{n-1} \geq 1\right)$. Em ambos os casos, $w$ é um produto de elementos de $\tilde{\mathbf{R}}$ e, portanto, pertence a $\tilde{\mathbf{A}}$. A independência linear segue dos mesmos argumentos utilizados em [36].

Estamos trabalhando com produtos de monômios quasi-standard. Podemos reordenálos através de argumentos como em PBW, onde é fixada uma ordem total, obedecendo ao comprimento $n$ desses monômios. Nesse processo, produtos entre $p$ monômios de mesmo comprimento desaparecem, uma vez que

$$
\left(r_{n-1}^{(1)} v_{n}\right) \cdot\left(r_{n-1}^{(2)} v_{n}\right) \cdots\left(r_{n-1}^{(p)} v_{n}\right)=r_{n-1} v_{n}^{p}=-r_{n-1} x_{n+1}^{p-1} v_{n+2} .
$$

Obtemos, então, produtos de elementos quasi-standard (no máximo $p-1$ para cada comprimento fixado), escritos em ordem decrescente de comprimento. Obtemos, por exemplo, um produto

$\left[\left(r_{n-1}^{(1)} v_{n}\right) \cdots\left(r_{n-1}^{(p-1)} v_{n}\right)\right] \cdot\left[\left(r_{n-2}^{(1)} v_{n-1}\right) \cdots\left(r_{n-2}^{(p-1)} v_{n-1}\right)\right] \cdots\left[\left(r_{0}^{(1)} v_{1}\right) \cdots\left(r_{0}^{(p-1)} v_{1}\right)\right] \cdot\left[v_{0} \cdots v_{0}\right]$

onde há pelo menos um monômio quasi-standard de comprimento $n$, enquanto os demais, de comprimentos $n-1, \ldots, 0$, podem ou não aparecer.

Podemos mover todas as letras $x_{i}$ em (5.9) para a esquerda. De fato, seja $x_{i}$ uma variável $x_{i}$ num monômio quasi-standard $r_{j-1} v_{j}(i<j)$. Os monômios quasi-standard que o antecedem em (5.9) têm comprimentos maiores que $j$ e, portanto, maiores que 
$i$. Logo, $x_{i}$ comuta com as cabeças $v_{k}$ correspondentes $(k>i)$. No caso em que o produto (5.9) envolve apenas um monômio quasi-standard $r_{n-1} v_{n}$ de comprimento $n$ e nenhum monômio de comprimento $n-1$, pelo menos uma das variáveis $\left\{x_{n-2}, x_{n-1}\right\}$ deve aparecer em grau no máximo $p-2$, visto que não é possível obter alguma dessas variáveis através de produtos com monômios de comprimentos menores ou iguais a $n-2$. Dessa forma, obtemos a condição $\xi_{n-2}+\xi_{n-1} \leq 2(p-1)-1$ se $\alpha_{n}=1 \mathrm{e}$ $\alpha_{n-1}=0$. Já no caso em que o produto (5.9) envolve pelo menos dois monômios de comprimento $n$, as variáveis $\left\{x_{n-2}, x_{n-1}\right\}$ podem aparecer em grau até $p-1$. O mesmo acontece para o caso em que pelo menos um monômio de comprimento $n-1$ aparece em (5.9). Provamos, portanto, a primeira afirmação.

A segunda afirmação é trivial, uma vez que $\tilde{\mathbf{A}}=\operatorname{Alg}(\tilde{\mathbf{R}}), \mathbf{A}=\operatorname{Alg}(\mathbf{R})$ e $\tilde{\mathbf{R}} \supset \mathbf{R}$. A terceira afirmação decorre do fato de que $r_{n-2} x_{n-1}^{\xi_{n-1}} v_{n}, v_{n}, v_{n-1}, \ldots, v_{0} \in \mathbf{R} \subset \mathbf{A}$ para $0 \leq \xi_{n-1} \leq p-2$.

Decorre das estimativas obtidas no Lema 5.19 que o peso de um monômio standard é sempre um inteiro positivo. Podemos generalizar essa afirmação para monômios quasi-standard.

Lema 5.25 Seja w um monômio quasi-standard. Então $\operatorname{wt}(w) \geq 1$.

Demonstração: Se $w$ tem comprimento 0 ou 1, a verificação é imediata, visto que $\operatorname{wt}\left(v_{0}\right)=1 \operatorname{e} \operatorname{wt}\left(x_{0}^{\xi_{0}} v_{1}\right) \geq p-(p-1)=1$. Fixemos então um comprimento $n \geq 2$. Observe que o monômio $u=x_{0}^{p-1} x_{1}^{p-1} \cdots x_{n-2}^{p-1} x_{n-1}^{p-1} v_{n}$ (que não é quasi-standard) tem peso

$$
\operatorname{wt}(u)=p^{n}-(p-1)\left(p^{0}+\cdots+p^{n-1}\right)=p^{n}-\left(p^{n}-1\right)=1 .
$$

Assim, se $w$ é um monômio quasi-standard de comprimento $n \geq 2$, pelo menos uma das variáveis $\left\{x_{n-2}, x_{n-1}\right\}$ deve aparecer em grau menor ou igual a $p-2$. Caso $w$ contenha no máximo $p-2$ letras $x_{n-2}$, seu peso satisfará wt $(w) \geq 1+p^{n-2}>1$. Por outro lado, caso $w$ contenha no máximo $p-2$ letras $x_{n-1}$, teremos $\operatorname{wt}(w) \geq 1+p^{n-1}>1$.

O próximo lema nos dá estimativas para pesos e superpesos de monômios da base de $\tilde{\mathbf{A}}$ obtida no Teorema 5.24 .

Lema 5.26 Seja $w=x_{0}^{\xi_{0}} \cdots x_{n-2}^{\xi_{n-2}} x_{n-1}^{\xi_{n-1}} v_{n}^{\alpha_{n}} v_{n-1}^{\alpha_{n-1}} v_{n-2}^{\alpha_{n-2}} \cdots v_{0}^{\alpha_{0}}$ um monômio pertencente a $\tilde{\mathbf{A}}$, com $n \geq 0$, nas mesmas condições do Teorema 5.24. O peso e o superpeso de $w$ 
satisfazem as desigualdades

$$
p^{n-2}<\operatorname{wt}(w)<p^{n+1}, \quad|\operatorname{swt}(w)| \leq(n+1)(p-1) .
$$

Demonstração: Para $n \leq 1$, essas estimativas podem ser facilmente verificadas. Tomemos então $n \geq 2$. Provaremos primeiramente as desigualdades referentes à função peso. Se $\alpha_{n}=1$ e $\alpha_{n-1} \geq 0$, a parte inicial de $w$ é um monômio quasi-standard $u=x_{0}^{\xi_{0}} \cdots x_{n-2}^{\xi_{n-2}} x_{n-1}^{\xi_{n-1}} v_{n}$ cujo peso satisfaz $1+p^{n-2} \leq \mathrm{wt}(u) \leq p^{n}$, conforme a demonstração do Lema 5.25. Assim, o peso de $w=u \cdot v_{n-2}^{\alpha_{n-2}} \cdots v_{0}^{\alpha_{0}}$ satisfaz

$$
p^{n-2}+1 \leq \operatorname{wt}(w) \leq p^{n}+(p-1)\left(p^{n-2}+\cdots+p+1\right)=p^{n}+p^{n-1}-1
$$

Se $\alpha_{n} \geq 2$, temos

$p^{n}+1=2 p^{n}-(p-1)\left(1+p+\cdots+p^{n-1}\right) \leq \operatorname{wt}(w) \leq(p-1)\left(p^{n}+\cdots+p+1\right)=p^{n+1}-1$.

Por fim, se $\alpha_{n-1} \geq 1$, temos

$p^{n-1}+1=p^{n}+p^{n-1}-(p-1)\left(1+p+\cdots+p^{n-1}\right) \leq \operatorname{wt}(w) \leq(p-1)\left(p^{n}+\cdots+p+1\right)=p^{n+1}-1$.

Em qualquer caso, valem as desigualdades $p^{n-2}<\operatorname{wt}(w)<p^{n+1}$.

Agora, a fim de limitarmos os superpesos, vamos supor primeiramente que $n$ seja par, com $n=2 k$. Uma vez que

$$
\begin{gathered}
-k(p-1) \leq \operatorname{swt}\left(x_{0}^{\xi_{0}} \cdots x_{2 k-1}^{\xi_{2 k-1}}\right) \leq k(p-1), \\
1-k(p-1) \leq \operatorname{swt}\left(v_{2 k}^{\alpha_{2 k}} v_{2 k-1}^{\alpha_{2 k-1}} \cdots v_{0}^{\alpha_{0}}\right) \leq(k+1)(p-1),
\end{gathered}
$$

temos $-2 k(p-1)+1 \leq \operatorname{swt}(w) \leq(2 k+1)(p-1)$, ou seja,

$$
-n(p-1) \leq \operatorname{swt}(w) \leq(n+1)(p-1)
$$

No caso em que $n$ é ímpar, com $n=2 k+1$, temos

$$
\begin{gathered}
-(k+1)(p-1) \leq \operatorname{swt}\left(x_{0}^{\xi_{0}} \cdots x_{2 k}^{\xi_{2 k}}\right) \leq k(p-1) \\
-(k+1)(p-1) \leq \operatorname{swt}\left(v_{2 k+1}^{\alpha_{2 k+1}} v_{2 k}^{\alpha_{2 k}} \cdots v_{0}^{\alpha_{0}}\right) \leq-1+(k+1)(p-1)
\end{gathered}
$$


e, portanto, $-(2 k+2)(p-1) \leq \operatorname{swt}(w) \leq(2 k+1)(p-1)-1$. Logo,

$$
-(n+1)(p-1) \leq \operatorname{swt}(w) \leq n(p-1)-1 .
$$

Em ambos os casos, vale a limitação $|\operatorname{swt}(w)| \leq(n+1)(p-1)$.

Com as estimativas encontradas no Lema 5.26, obtemos informações a respeito da representação geométrica dos monômios de $\mathbf{A}=\operatorname{Alg}(\mathbf{R})$ no plano $\mathbb{R}^{2}$ em termos das coordenadas peso $\left(Z_{1}, Z_{2}\right)$.

Teorema 5.27 O conjunto dos pontos do plano associados aos monômios de $\mathbf{A}=$ $\mathrm{Alg}(\mathbf{R})$ é limitado por duas curvas logarítmicas em termos das coordenadas peso $\mathrm{Wt}(w)=\left(Z_{1}, Z_{2}\right)$ :

$$
\left|Z_{2}\right|<\log _{2} Z_{1}+2
$$

Demonstração: Seja $w=x_{0}^{\xi_{0}} \cdots x_{n-2}^{\xi_{n-2}} x_{n-1}^{\xi_{n-1}} v_{n}^{\alpha_{n}} v_{n-1}^{\alpha_{n-1}} v_{n-2}^{\alpha_{n-2}} \cdots v_{0}^{\alpha_{0}}$ um monômio pertencente a $\mathbf{A}=\operatorname{Alg}(\mathbf{R}) \subset \tilde{\mathbf{A}}$. Pelo Lema 5.26, valem as desigualdades

$$
p^{n-2}<Z_{1} \quad \text { e } \quad\left|Z_{2}\right| \leq(n+1)(p-1),
$$

de onde concluímos que $\left|Z_{2}\right|<\left(\log _{p} Z_{1}+3\right)(p-1)$.

Com as limitações encontradas no Lema 5.26, podemos estimar o crescimento da álgebra $\mathbf{A}=\operatorname{Alg}(\mathbf{R})$ e calcular sua dimensão de Gelfand-Kirillov.

Teorema 5.28 Seja $\mathbf{A}=\operatorname{Alg}\left(v_{0}, v_{1}\right)$. Valem as seguintes afirmações:

(i) Existem constantes $c_{1}, c_{2}>0$ tais que $c_{1} m^{2} \leq \tilde{\gamma}_{\mathbf{A}}(m) \leq c_{2} m^{2}$;

(ii) Existem constantes $c_{3}, c_{4}>0$ tais que $c_{1} m^{2} \leq \gamma_{\mathbf{A}}(m) \leq c_{4} m^{2}$;

(iii) $\mathrm{GKdim} \mathbf{A}=\underline{\mathrm{GK} \operatorname{dim}} \mathbf{A}=2$.

Demonstração: Fixemos um número natural $m \geq p$ e façamos $n=\left[\log _{p} m\right]-1 \geq$ 0. Consideremos monômios $w=x_{0}^{\xi_{0}} \cdots x_{n-2}^{\xi_{n-2}} x_{n-1}^{\xi_{n-1}} v_{n}^{\alpha_{n}} v_{n-1}^{\alpha_{n-1}} v_{n-2}^{\alpha_{n-2}} \cdots v_{0}^{\alpha_{0}} \in \tilde{\mathbf{A}}$ como no Teorema 5.24, com $\xi_{n-1} \leq p-2$. Esses monômios pertencem a $\mathbf{A}$ e, pelo Lema 5.26, 
temos $\operatorname{wt}(w)<p^{n+1} \leq m$. O número desses monômios constitui, portanto, uma cota inferior para $\tilde{\gamma}_{\mathbf{A}}(m)$. Temos então

$$
\begin{aligned}
\tilde{\gamma}_{\mathbf{A}}(m) & \geq p^{n-1} \cdot(p-1) \cdot(p-1) \cdot p^{n}=p^{2 n-1} \cdot(p-1)^{2} \\
& \geq p^{2 n-1} \geq p^{2 \log _{p} m-5}=p^{\log _{p} m^{2}-5}=\frac{1}{p^{5}} m^{2}
\end{aligned}
$$

Fixemos novamente um número natural $m$ e façamos $n=\left[\log _{p} m\right]+2 \geq 2$. Considere agora um monômio qualquer $w=x_{0}^{\xi_{0}} \cdots x_{n-2}^{\xi_{n-2}} x_{j-1}^{\xi_{j-1}} v_{j}^{\alpha_{j}} v_{j-1}^{\alpha_{j-1}} \cdots v_{0}^{\alpha_{0}}$ pertencente à base de $\tilde{\mathbf{A}}$ determinada no Teorema 5.24. Se $j>n$, então $\operatorname{wt}(w)>p^{j-1}>p^{n-1}>m$. Para que tenhamos $\operatorname{wt}(w) \leq m$, é necessário que se tenha $j \leq n$. Então, o número desses monômios $w$ com $j \leq n$ nos dá uma cota superior para $\tilde{\gamma}_{\mathbf{A}}(m)$.

Nessas condições, para $j=0$, temos apenas o monômio $w=v_{0}$. Para $j=1$, temos $p^{2}(p-1)$ monômios possíveis, pois $w \in \tilde{\mathbf{A}}$ é da forma $w=x_{0}^{\xi_{0}} v_{1}^{\alpha_{1}} v_{0}^{\alpha_{0}}$, onde $0 \leq \xi_{0}, \alpha_{0} \leq$ $p-1$ e $1 \leq \alpha_{1} \leq p-1$. Suponhamos então $j \geq 2$. O número desses monômios com $\alpha_{j}=1$ e $\alpha_{j-1}=0$ é $p^{j}(p-1)^{j-1}-p^{j-2}(p-1)^{j-1}=\left(p^{j}-p^{j-2}\right)(p-1)^{j-1}$, visto que as letras $\left\{x_{j-2}, x_{j-1}\right\}$ não podem aparecer em grau $p-1$ simultaneamente. Por outro lado, o número desses monômios $w$ com $\alpha_{j} \geq 2$ é $p^{j}(p-2) p^{j}=p^{2 j}(p-2)$ e o número desses monômios $w$ com $\alpha_{j-1} \geq 1$ é $p^{j}(p-1)^{2} p^{j-1}=p^{2 j-1}(p-1)$. Portanto,

$$
\begin{aligned}
\tilde{\gamma}_{\mathbf{A}}(m) & \leq 1+p^{2}(p-1)+\sum_{j=2}^{n}\left[\left(p^{j}-p^{j-2}\right)(p-1)^{j-1}+p^{2 j}(p-2)+p^{2 j-1}(p-1)\right] \\
& \leq 1+p^{2}(p-1)+\sum_{j=2}^{n}\left[p^{2 j-1}+p^{2 j+1}+p^{2 j}\right] \\
& \leq 1+p^{2}(p-1)+\sum_{j=2}^{n} 2 p^{j+1} \leq 1+p^{2}(p-1)+2 p^{5} \cdot \frac{p^{2 n-2}-1}{p^{2}-1} \\
& \leq 1+p^{2}(p-1)+2\left(p^{6}-p^{4}+p^{2}-1\right) \cdot \frac{p^{2 n-2}-1}{p^{2}-1} \\
& \leq 1+p^{3}-p^{2}+2\left(p^{4}+1\right)\left(p^{2 n-2}-1\right) \leq p^{4}+\left(p^{4}+1\right) \cdot p^{2 n-1} \\
& \leq p^{5} \cdot p^{2 n-1}=p^{2 n+4} \leq p^{2 \log _{p} m+4}=p^{4} \cdot m^{2} .
\end{aligned}
$$

Dessas desigualdades e de (5.10), verifica-se as afirmações (i) e (iii). Para a prova de (ii), basta considerarmos o mesmo argumento utilizado no Corolário 4.27. 


\subsection{As componentes homogêneas da $\mathbb{Z}^{2}$-graduação de $\mathrm{R}$ são no máximo unidimensionais}

Vimos na Seção 5.4 que $\mathbf{R}$ possui uma $\mathbb{Z}^{2}$-graduação pelo multigrau em relação a $\left\{v_{0}, v_{1}\right\}$. Nesta seção, mostraremos que as componentes homogêneas dessa graduação são uniformemente limitadas. Para isso, precisamos dos dois lemas a seguir.

Lema 5.29 Seja $T_{n}^{k}$ o conjunto dos monômios standard de comprimento $n \geq 2$ e tipo $k \in\{1,2\}$. Então

$$
T_{n+1}^{1}=\left\{1, x_{0}, \ldots, x_{0}^{p-1}\right\} \cdot \tau\left(T_{n}^{1}\right) ; \quad T_{n+1}^{2}=\tau\left(T_{n}^{2}\right)
$$

Demonstração: Segue imediatamente da estrutura dos monômios standard.

Lema 5.30 Se u e v são monômios standard distintos, ambos de comprimento menor ou igual a 2 , então $\operatorname{Gr}(u) \neq \operatorname{Gr}(v)$.

Demonstração: Observe inicialmente que $\operatorname{Gr}\left(v_{0}\right)=(1,0)$ e $\operatorname{Gr}\left(v_{1}\right)=(0,1)$ são os multigraus dos monômios standard de comprimento 0 e 1 , respectivamente. Denotando por $T_{2}^{1}=\left\{x_{0}^{\xi_{0}} x_{1}^{\xi_{1}} v_{2} \mid 0 \leq \xi_{0} \leq p-1,0 \leq \xi_{1} \leq p-2\right\}$ o conjunto dos monômios standard do primeiro tipo de comprimento 2 e fazendo $X=\left\{\left(X_{1}, X_{2}\right) \mid 1 \leq X_{1} \leq p, 1 \leq X_{2} \leq\right.$ $p-1\}$, temos a seguinte correspondência biunívoca:

$$
\begin{array}{ccc}
T_{2}^{1} & \longrightarrow & X \\
x_{0}^{\xi_{0}} x_{1}^{\xi_{1}} v_{2} & \longmapsto & \left(X_{1}, X_{2}\right)=\left(p-\xi_{0}, p-1-\xi_{1}\right) .
\end{array}
$$

Além disso, o único monômio standard do segundo tipo de comprimento 2 tem multigrau $\operatorname{Gr}\left(x_{1}^{p-1} v_{2}\right)=\operatorname{Gr}\left(v_{2}\right)+\operatorname{Gr}\left(x_{1}^{p-1}\right)=(p, p-1)-(0, p-1)=(p, 0) \notin X$. Isso conclui a demonstração.

Como consequência do Lema 5.29 e do Lema 5.30, temos o seguinte resultado:

Teorema 5.31 As componentes da $\mathbb{Z}^{2}$-graduação de $\mathbf{R}=\operatorname{Lie}_{p}\left(v_{0}, v_{1}\right)$ obtida no Lema 5.10 são no máximo unidimensionais.

Demonstração: Provaremos este resultado por contradição. Suponhamos que existam dois monômios standard distintos $u, v$ com o mesmo multigrau $\operatorname{Gr}(u)=\operatorname{Gr}(v)=$ 
$\left(X_{1}, X_{2}\right)$ (e, consequentemente, com o mesmo vetor peso $\left.\mathrm{Wt}(u)=\mathrm{Wt}(v)=\left(Z_{1}, Z_{2}\right)\right)$. Pelo Lema 5.30, os comprimentos de $u$ e $v$ não podem ser menores ou iguais a 2 simultaneamente. Sem perda de generalidade, podemos considerar que $u$ tem comprimento maior ou igual a 3 . Assim, pelo Lema 5.19, temos $\operatorname{wt}(u) \geq p^{2}$. Uma vez que $\operatorname{wt}(v)=\operatorname{wt}(u)$, do Lema 5.19 inferimos também que o comprimento de $v$ deve ser maior ou igual a 2 .

Afirmamos que o comprimento de $v$ não é igual a 2 . Se o fosse, teríamos $\operatorname{wt}(v)=$ $\operatorname{wt}(u)=p^{2}$ e, consequentemente, $v=v_{2}$ e $u=x_{2}^{p-1} v_{3}$. Mas $\operatorname{swt}\left(v_{2}\right)=1 \neq-p=$ $\operatorname{swt}\left(x_{2}^{p-1} v_{3}\right)$. Isso contradiz a nossa hipótese de que $u$ e $v$ têm o mesmo multigrau e, consequentemente, o mesmo vetor peso. Portanto, os comprimentos de $u$ e $v$ são, ambos, maiores ou iguais a 3. Podemos supor que o menor desses comprimentos é minimal sob a condição de termos $u \neq v$ com o mesmo multigrau. Pelo Lema 5.29, temos

$$
u=x_{0}^{\alpha_{0}} \tau(\tilde{u}), \quad v=x_{0}^{\beta_{0}} \tau(\tilde{v}) ; \quad \alpha_{0}, \beta_{0} \in\{0, \ldots, p-1\},
$$

onde $\tilde{u}, \tilde{v}$ são monômios standard cujos comprimentos são inferiores, por uma unidade, aos comprimentos de $u$ e $v$, respectivamente. Sejam $\operatorname{Gr}(\tilde{u})=\left(n_{1}, n_{2}\right)$ e $\operatorname{Gr}(\tilde{v})=$ $\left(m_{1}, m_{2}\right)$. Através do Lema 5.14, chegamos à seguinte relação:

$$
A^{T} \cdot\left(\begin{array}{c}
n_{1} \\
n_{2}
\end{array}\right)-\left(\begin{array}{c}
\alpha_{0} \\
0
\end{array}\right)=A^{T} \cdot\left(\begin{array}{c}
m_{1} \\
m_{2}
\end{array}\right)-\left(\begin{array}{c}
\beta_{0} \\
0
\end{array}\right)=\left(\begin{array}{c}
X_{1} \\
X_{2}
\end{array}\right),
$$

onde $A=\left(\begin{array}{cc}0 & 1 \\ p & p-1\end{array}\right)$. Temos então

$$
\begin{aligned}
\left(\begin{array}{c}
n_{1}-m_{1} \\
n_{2}-m_{2}
\end{array}\right) & =\left(A^{T}\right)^{-1}\left(\begin{array}{c}
\alpha_{0}-\beta_{0} \\
0
\end{array}\right) \\
& =\frac{1}{p}\left(\begin{array}{cc}
1-p & p \\
1 & 0
\end{array}\right) \cdot\left(\begin{array}{c}
\alpha_{0}-\beta_{0} \\
0
\end{array}\right) \\
& =\frac{1}{p}\left(\begin{array}{c}
(1-p)\left(\alpha_{0}-\beta_{0}\right) \\
\alpha_{0}-\beta_{0}
\end{array}\right) .
\end{aligned}
$$

Como $\alpha_{0}, \beta_{0} \in\{0, \ldots, p-1\}$, temos $\left|\alpha_{0}-\beta_{0}\right| \in\{0, \ldots, p-1\}$. Uma vez que $n_{1}-m_{1}=\frac{1-p}{p}\left(\alpha_{0}-\beta_{0}\right)$ é um número inteiro, necessariamente temos $\left|\alpha_{0}-\beta_{0}\right|=0$, isto é, $\alpha_{0}=\beta_{0}$. Portanto, $\left(n_{1}, n_{2}\right)=\left(m_{1}, m_{2}\right)$, isto é, $\operatorname{Gr}(\tilde{u})=\operatorname{Gr}(\tilde{v})$. Pela minimalidade do exemplo, segue que $\tilde{u}=\tilde{v}$. Uma vez que $\alpha_{0}=\beta_{0}$, temos 
$u=x_{0}^{\alpha_{0}} \tau(\tilde{u})=x_{0}^{\beta_{0}} \tau(\tilde{v})=v$ e chegamos numa contradição, o que prova o resultado.

Provaremos no resultado a seguir que $\mathbf{R}$ não tem uma p-aplicação nil.

Lema 5.32 Valem as seguintes afirmações:

(i) A p-aplicação definida na álgebra de Lie restrita $\mathbf{R}=\operatorname{Lie}_{p}\left(v_{0}, v_{1}\right)$ não é nil.

(ii) A álgebra A não é nil.

Demonstração: Para $n \geq 0$ e $0 \neq \alpha \in K$, consideremos o elemento não nulo $v=$ $v_{n}+\alpha x_{n}^{p-1} v_{n+1} \in \mathbf{R}$. Temos

$$
\begin{aligned}
v^{p} & =\left(v_{n}+\alpha x_{n}^{p-1} v_{n+1}\right)^{p}=v_{n}^{p}+\left(\operatorname{ad} v_{n}\right)^{p-1}\left(\alpha x_{n}^{p-1} v_{n+1}\right) \\
& =-x_{n+1}^{p-1} v_{n+2}-\alpha v_{n+1} \\
& =-\alpha\left(v_{n+1}+\frac{1}{\alpha} x_{n+1}^{p-1} v_{n+2}\right)
\end{aligned}
$$

é um elemento não nulo. Observe que $v_{n+1}+\frac{1}{\alpha} x_{n+1}^{p-1} v_{n+2}$ tem uma forma semelhante à de $v$. Assim, temos

$$
v^{p^{2}}=-\alpha^{p}\left(-\frac{1}{\alpha}\right)\left(v_{n+2}+\alpha x_{n+2}^{p-1} v_{n+3}\right)=\alpha^{p-1}\left(v_{n+2}+\alpha x_{n+2}^{p-1} v_{n+3}\right),
$$

onde $v_{n+2}+\alpha x_{n+2}^{p-1} v_{n+3}$ também é não nulo e tem forma semelhante a $v$. Efetuando a $p$-aplicação repetidas vezes sobre $v$, sempre vamos obter um elemento não nulo, da forma $\alpha^{r}\left(v_{m}+\alpha^{ \pm 1} x_{m}^{p-1} v_{m+1}\right)$. Concluímos assim que $\mathbf{R}$ não tem $p$-aplicação nil.

Teorema 5.33 $\mathrm{Na}$ decomposição triangular $\mathbf{A}=\mathbf{A}_{+} \oplus \mathbf{A}_{0} \oplus \mathbf{A}_{-}$, as subálgebras $\mathbf{A}_{+}$ e $\mathbf{A}_{-}$são localmente nilpotentes.

Demonstração: Segue dos argumentos utilizados no artigo [36].

\subsection{Just infinitude da álgebra de Lie restrita $R$}

No Teorema 5.6, mostramos que a álgebra de Lie restrita $\mathbf{R}=\operatorname{Lie}_{p}\left(v_{0}, v_{1}\right)$ tem dimensão infinita. Contudo, ao tomarmos quocientes dessa álgebra por ideais não nulos, obtemos álgebras de dimensão finita. Provaremos esse fato no teorema a seguir. 
Teorema 5.34 A álgebra de Lie restrita $\mathbf{R}=\operatorname{Lie}_{p}\left(v_{0}, v_{1}\right)$ é uma álgebra just infinite.

Demonstração: No Teorema 5.6, exibimos uma base de $\mathbf{R}$ composta por infinitos monômios standard, mostrando que $\operatorname{dim}_{K} \mathbf{R}=\infty$. Consideremos agora $J \triangleleft \mathbf{R}$ um ideal bilateral não nulo da álgebra de Lie restrita $\mathbf{R}$ e seja $0 \neq w \in J$.

Nosso objetivo agora é encontrar um elemento $v \neq 0$ do ideal $J \triangleleft \mathbf{R}$ tal que, escrito como combinação linear dos monômios standard com coeficientes não nulos, ao menos um desses monômios não contenha letras $x_{i}$. Uma vez que $w \in \mathbf{R}$, podemos escrevêlo como combinação linear finita de monômios standard com coeficientes não nulos. Dentre esses monômios standard, há no máximo dois com peso maximal (Teorema 5.21). Assim, escrevemos

$$
w=\tilde{w}+\alpha w_{1}+\beta w_{2}
$$

de forma que $\tilde{w}$ seja combinação linear finita de monômios standard de peso inferior a $\operatorname{wt}\left(w_{1}\right)=\operatorname{wt}\left(w_{2}\right)$, onde $w_{1}$ e $w_{2}$ são, respectivamente, monômios standard do primeiro e do segundo tipo e, além disso, pelo menos um dos coeficientes $\{\alpha, \beta\}$ é não nulo. Vamos analisar os dois casos possíveis.

1) Se $\alpha \neq 0$ e $\beta \neq 0$ em (5.11), tem-se necessariamente $\operatorname{wt}\left(w_{1}\right)=\operatorname{wt}\left(w_{2}\right)=p^{k}$ para algum $k \geq 1$, de onde concluímos que $w_{1}=v_{k}$ e $w_{2}=x_{k}^{p-1} v_{k+1}$ e, assim,

$$
w=\tilde{w}+\alpha v_{k}+\beta x_{k}^{p-1} v_{k+1} .
$$

Nesse caso, uma vez que $v_{k}$ não contém letras $x_{i}$, podemos tomar $v=w$.

2) Se $\alpha=0$ ou $\beta=0$, temos

$$
w=\tilde{w}+\lambda u,
$$

onde $u=x_{0}^{\xi_{0}} \cdots x_{N-1}^{\xi_{N-1}} v_{N}$ e $\lambda \neq 0$ (se $\alpha=0$, fazemos $\lambda=\beta$ e $u=w_{2}$; se $\beta=0$, fazemos $\lambda=\alpha$ e $\left.u=w_{1}\right)$. Multiplicando $w$ à esquerda sucessivamente por cada elemento pivô $v_{i}(0 \leq i \leq N-1)$ em grau $\xi_{i}$, eliminamos todas as letras $x_{i}$ de $u$. Obtemos então um novo elemento não nulo

$$
v=w^{\prime}+\lambda v_{N}
$$

onde $w^{\prime}$ é combinação linear de monômios standard com peso inferior a $\operatorname{wt}\left(v_{N}\right)=p^{N}$. Observe que o termo $\lambda v_{N}(0 \neq \lambda \in K)$ não contém letras $x_{i}$, como desejávamos.

O elemento $0 \neq v \in J$ obtido é uma combinação linear de monômios standard. Consideremos $m$ o maior comprimento dentre os monômios standard que aparecem nessa combinação linear com coeficiente não nulo, e seja $I$ o conjunto dos índices $i \in\{0, \ldots, m\}$ 
para os quais essa combinação contém um termo da forma $\lambda_{i} v_{i}, 0 \neq \lambda_{i} \in K$. Multiplicando $v$ à direita pelo elemento $x_{0}^{p-1} \cdots x_{m}^{p-1} v_{m+2} \in \mathbf{R}$, anulamos todos os termos que contêm alguma letra $x_{i}$ e obtemos

$$
\left[v, x_{0}^{p-1} \cdots x_{m}^{p-1} v_{m+2}\right]=\sum_{i \in I} \lambda_{i} x_{0}^{p-1} \cdots x_{i-1}^{p-1} x_{i}^{p-2} x_{i+1}^{p-1} \cdots x_{m}^{p-1} v_{m+2}
$$

Observe que as variáveis $x_{i}$ que aparecem em grau $p-1$ em todos os termos de (5.12) são exatamente as letras $x_{i}$ tais que $i \in \bar{I}=\{0, \ldots, m\} \backslash I$. Multiplicando (5.12) à esquerda sucessivamente por elementos pivô $v_{i} \operatorname{com} i \in \bar{I}$ em grau $p-1$, e por elementos pivô $v_{i}$ com $i \in I$ em grau $p-2$, obtemos, fazendo $I=\left\{i_{1}, \ldots, i_{k}\right\}\left(i_{1}<\ldots<i_{k}\right)$ :

$$
\sum_{j=1}^{k} \lambda_{i_{j}} x_{i_{1}} \cdots \widehat{x_{i_{j}}} \cdots x_{i_{k}} v_{m+2} \in J
$$

Agora multiplicamos (5.13) à esquerda sucessivamente pelos elementos pivô $v_{i_{1}}, v_{i_{2}}, \ldots, v_{i_{k-1}}$, resultando num elemento do tipo

$$
v^{\prime}+\lambda_{i_{k}} v_{m+2} \in J
$$

onde $v^{\prime}$ é uma combinação linear (possivelmente nula) de monômios standard de comprimento $m+2$ que contêm pelo menos uma letra $x_{i}$. Multiplicando (5.14) por $x_{0}^{p-1} \cdots x_{m+2}^{p-1} v_{m+4}$, anulamos $v^{\prime}$ e obtemos

$$
\left[v^{\prime}+\lambda_{i_{k}} v_{m+2}, x_{0}^{p-1} \cdots x_{m+2}^{p-1} v_{m+4}\right]=\lambda_{i_{k}} x_{0}^{p-1} \cdots x_{m+1}^{p-1} x_{m+2}^{p-2} v_{m+4} .
$$

Multiplicando (5.15) à esquerda sucessivamente por $v_{i}$ em grau $p-1(0 \leq i \leq m+1) \mathrm{e}$ depois por $v_{m+2}$ em grau $p-2$, eliminamos todas as letras $x_{i}$ e concluímos que $v_{m+4} \in J$. Pelo item (v) do Lema 5.1, $v_{m+5}=-\left[\left(v_{m+4}\right)_{p-2},\left(v_{m+3}\right)_{p}, v_{m+4}\right]$ também pertence ao ideal $J$. Usando o item (v) do Lema 5.1, uma simples indução nos faz concluir que $v_{n} \in J$ para qualquer $n \geq m+4$.

Vamos mostrar que todo monômio standard de comprimento $n \geq m+7$ pertence a $J$. Com efeito, se $n \geq m+7$, temos

$$
\left[v_{n-2}, x_{0}^{p-1} \cdots x_{n-3}^{p-1} v_{n-1}\right]=x_{0}^{p-1} \cdots x_{n-2}^{p-1} x_{n-1}^{p-2} v_{n} \in J
$$

Fazendo o produto comutador desse elemento por elementos pivô $v_{i}, i \in\{0, \ldots, n-1\}$, podemos eliminar fatores $x_{i}$. Portanto, $J$ contém todos os monômios standard de 
comprimento $n \geq m+7$ do primeiro tipo.

Uma vez que $J$ é um ideal da álgebra de Lie restrita $\mathbf{R}, J$ absorve as potências de grau $p$ de seus elementos. Logo, $v_{i}^{p}=-x_{i+1}^{p-1} v_{i+2}$ para todo $i \geq m+4$. Daí concluímos que todo monômio standard do segundo tipo de comprimento $n \geq m+6$ pertence ao ideal $J$.

Provamos então que $J$ contém todos os monômios standard de comprimento maior ou igual a $m+7$. Portanto, a dimensão da álgebra quociente $\mathbf{R} / J$ é finita e não excede o número de monômios standard de comprimento menor ou igual a $m+6$. 


\section{Referências Bibliográficas}

[1] Bacher R., Determinants related to Dirichlet characters modulo 2, 4 and 8 of binomial coefficients and the algebra of recurrence matrices. Int. J. Algebra Comput. 18, (2008), no. 3, 535-566.

[2] Bahturin Yu.A., Mikhalev A.A., Petrogradsky V.M., and Zaicev M. V., Infinite dimensional Lie superalgebras, de Gruyter Exp. Math. vol. 7, de Gruyter, Berlin, 1992.

[3] Bahturin, Yu.A.; Olshanskii, A., Large restricted Lie algebras, J. Algebra (2007) 310, No. 1, 413-427.

[4] Bahturin, Yu.A.; Sehgal, S.K.; Zaicev, M.V., Group gradings on associative algebras, J. Algebra (2001) 241, No. 2, 677-698.

[5] Bartholdi L., Branch rings, thinned rings, tree enveloping rings. Israel J. Math. 154 (2006), 93-139.

[6] Bartholdi L., Self-similar Lie algebras. J. Eur. Math. Soc. (JEMS) 17 (2015), no. $12,3113-3151$.

[7] Bartholdi L., Grigorchuk R.I., Lie methods in growth of groups and groups of finite width. Computational and geometric aspects of modern algebra,1-27, London Math. Soc. Lecture Note Ser., 275, Cambridge Univ. Press, Cambridge, 2000.

[8] Bouarroudj, S., Grozman, P., Leites, D. Classification of finite dimensional modular Lie superalgebras with indecomposable Cartan matrix, SIGMA 5 (2009), 060, $1-63$.

[9] Drensky V. and Hammoudi L., Combinatorics of words and semigroup algebras which are sums of locally nilpotent subalgebras. Canad. Math. Bull. 47 (2004), no. $3,343-353$.

[10] Elduque, A. Fine gradings on simple classical Lie algebras. J. Algebra 324 (2010), No. 12 , 3532-3571. 
[11] Ershov M., Golod-Shafarevich groups: a survey, Int. J. Algebra Comput. 22, (2012) No. 5, Article ID 1230001.

[12] Fabrykowski, J., Gupta, N., On groups with sub-exponential growth functions. J. Indian Math. Soc. (N.S.) 49 (1985), no. 3-4, 249-256.

[13] Golod, E.S. On nil-algebras and finitely approximable p-groups. Am. Math. Soc., Translat., II. Ser. 48, 103-106 (1965); translation from Izv. Akad. Nauk SSSR, Ser. Mat. 28, 273-276 (1964).

[14] Golod E.S. and Shafarevich I.R., On the class field tower, Am. Math. Soc., Translat., II. Ser. 48, 91-102 (1965); translation from Izv. Akad. Nauk SSSR Ser. Mat. 28 (1964), 261-272;

[15] Golod, E.S. On some problems of Burnside type. Am. Math. Soc., Translat., II. Ser. 84, (1969) 83-88; translation from Tr. Mezdunarod. Kongr. Mat., Moskva 1966, 284-289 (1968).

[16] Grigorchuk, R.I., On the Burnside problem for periodic groups., Funktsional. Anal. i Prilozhen. 14 (1980), no. 1, 53-54.

[17] Grigorchuk, R.I. Degrees of growth of finitely generated groups, and the theory of invariant means. Math. USSR, Izv. 25 (1985), 259-300; translation from Izv. Akad. Nauk SSSR, Ser. Mat. 48 (1984), No.5, 939-985.

[18] Grigorchuk, R.I., Just infinite branch groups. New horizons in pro- $p$ groups, 121179, Progr. Math., 184, Birkhauser Boston, Boston, MA, 2000.

[19] Gupta N., and Sidki S., On the Burnside problem for periodic groups., Math. Z. 182 (1983), no. 3, 385-388.

[20] Humphreys J.E., Introdution to Lie algebras and representation theory, SpringerVerlag, New York. 1970.

[21] Jacobson N., Lie algebras, Interscience, New York. 1962.

[22] Iohara, K.; Mathieu, O., Classification of simple Lie algebras on a lattice. Proc. Lond. Math. Soc. (3) 106 (2013), no. 3, 508-564.

[23] Krause G.R. and Lenagan T.H., Growth of algebras and Gelfand-Kirillov dimension, AMS, Providence, R.I., 2000.

[24] Kac, V.G. Lie superalgebras. Adv. Math. 26, (1977), 8-96. 
[25] Kelarev A.V., A sum of two locally nilpotent rings may be not nil. Arch. Math. 60 (1993), no. 5, 431-435.

[26] Krylyouk Ia., The enveloping algebra of the Petrogradsky-Shestakov-Zelmanov algebra is not graded-nil in the critical characteristics, J. Lie Theory, 21, (2011), No. 3, 703-709.

[27] Lenagan, T.H., Smoktunowicz Agata., An infinite dimensional affine nil algebra with finite Gelfand-Kirillov dimension, J. Am. Math. Soc. 20, (2007) No. 4, 989 1001.

[28] Martinez C., Zelmanov E., Nil algebras and unipotent groups of finite width. Adv. Math. 147, (1999) No.2, 328-344.

[29] Mikhalev A.A., Subalgebras of free Lie $p$-superalgebras. Math. Notes 43, (1988) No.2, 99-106; translation from Mat. Zametki 43 (1988) No.2, 178-191.

[30] Musson I.M., Lie superalgebras and enveloping algebras, American Mathematical Society, Providence - Rhode Island, c2012.

[31] Passman D.S. and Petrogradsky V.M., Polycyclic restricted Lie algebras, Comm. Algebra, 29 (2001), no. 9, 3829-3838.

[32] Petrogradsky V.M., On Lie algebras with nonintegral q-dimensions. Proc. Amer. Math. Soc. 125 (1997), no. 3, 649-656.

[33] Petrogradsky V.M., Examples of self-iterating Lie algebras, J. Algebra, 302 (2006), no. $2,881-886$.

[34] Petrogradsky V.M., Fractal nil graded Lie superalgebras, J. Algebra, 466 (2016), 229-283.

[35] Petrogradsky V. M., Yu. P. Razmyslov, and E. O. Shishkin, Wreath products and Kaluzhnin-Krasner embedding for Lie algebras, Proc. Amer. Math. Soc., 135, (2007), 625-636.

[36] Petrogradsky V.M. and Shestakov I.P. Examples of self-iterating Lie algebras, 2, J. Lie Theory, 19 (2009), no. 4, 697-724.

[37] Petrogradsky V.M. and Shestakov I.P. Self-similar associative algebras, J. Algebra, 390 (2013), 100-125.

[38] Petrogradsky V.M. and Shestakov I.P. On properties of Fibonacci restricted Lie algebra, J. Lie Theory, 23 (2013), no. 2, 407-431. 
[39] Petrogradsky V.M., Shestakov I.P., and Zelmanov E., Nil graded self-similar algebras, Groups Geom. Dyn., 4 (2010), no. 4, 873-900.

[40] Petrogradsky V.M., Nil Lie p-algebras of slow growth, Comm. Algebra.

[41] Radford D. E., Divided power structures on Hopf algebras and embedding Lie algebras into special-derivation algebras, J. Algebra, 98 (1986), 143-170.

[42] Yu.P. Razmyslov, Identities of algebras and their representations. AMS, Providence RI 1994.

[43] Rozhkov, A.V. Lower central series of a group of tree automorphisms, Math. Notes 60, No.2, 165-174 (1996); translation from Mat. Zametki 60, No.2, 225-237 (1996).

[44] Shestakov I.P. and Zelmanov E., Some examples of nil Lie algebras. J. Eur. Math. Soc. (JEMS) 10 (2008), no. 2, 391-398.

[45] Sidki S.N., Functionally recursive rings of matrices - Two examples. J.Algebra 322 (2009), no. 12, 4408-4429.

[46] Sidki S.N., A primitive ring associated to a Burnside 3-group. J. London Math. Soc. (2) 55 (1997), no. 1, 55-64.

[47] Scheunert, M. The theory of Lie superalgebras. Lecture Notes in Mathematics. 716. Berlin. Springer-Verlag. 1979.

[48] Strade H., Simple Lie algebras over fields of positive characteristic. I: Structure theory. Berlin: de Gruyter. 2004.

[49] Strade H. and Farnsteiner R., Modular Lie algebras and their representations, New York etc.: Marcel Dekker, 1988.

[50] Voden T., Subalgebras of Golod-Shafarevich algebras, Int. J. Algebra Comput. 19, (2009) No. 3, 423-442.

[51] Zelmanov E., Some open problems in the theory of infinite dimensional algebras., J. Korean Math. Soc. 44 (2007), no. 5, 1185-1195. 